\title{
Fit States on Girard Algebras
}

\author{
Remigijus Petras Gylys ${ }^{1}$ \\ ${ }^{1}$ Vilnius University Institute of Mathematics and Informatics, Akademijos 4, Vilnius, Lithuania \\ Correspondence: Remigijus Petras Gylys, Vilnius University Institute of Mathematics and Informatics, Akademi- \\ jos 4, Vilnius 08663, Lithuania. E-mail: gyliene@ktl.mii.lt
}

Received: July 18, 2014 Accepted: August 1, 2014 Online Published: October 9, 2014

doi:10.5539/jmr.v6n4p29 URL: http://dx.doi.org/10.5539/jmr.v6n4p29

\begin{abstract}
Recently Weber proposed to define "weakly additive" states on a Girard algebra by the additivity only on its sub$M V$-algebras and characterized such states on the canonical Girard algebra extensions of any finite $M V$-chain. In the present paper, we take another viewpoint: the arguable sub- $M V$-algebras are replaced by suitable substructures coming from author, Höhle and Weber's own previous investigations. We propose a new notion of fit states on a Girard algebra by the additivity on the mentioned substructures and consider such states on the "non-effectible" Girard algebra " $n$-extensions" (= canonical extensions when $n=1$ ) of $M V$-chains restricting ourselves to ones having less than six nontrivial elements. Our fit states appear as solutions of certain inconsistent systems of linear equations. They have extensive enough domains of the additivity-in any comparable case more extensive than Weber's states have.
\end{abstract}

Keywords: $M V$-algebra, Weber $M V$-chain, Girard algebra, effectible Girard algebra, canonical extension, $n$ extension, steady product, deflected product, state, fit state, Weber state

\section{Introduction}

Höhle and Weber (1997) proposed the following notion of an additivity of a state on a Girard algebra $\left(Q ; \wp, \wp^{-}\right)$ (with the dual multiplication $\wp$ viewed as an addition and its dual residual $\wp^{-}$): an isotonic map st: $Q \rightarrow[0,1]$ satisfying boundary conditions is additive whenever

$$
\wp \frac{x \wp y}{x}=y \text { and } \wp \frac{x \wp y}{y}=x \Rightarrow \operatorname{st}(x \wp y)=\operatorname{st}(x)+\operatorname{st}(y),
$$

where the antecedent of the implication hints at a possible new concept of disjointness in $Q$ (reducing to the usual one in $M V$-algebras).

Let us remember what happened to this additivity in such a delicate situation when the authors themselves keep this notion in the mind unwillingly. Weber (2010) proved the uniqueness of an additive state on a finite non-Boolean $M V$-chain, say $\mathcal{W}_{m}$, having $m-1$ (with $m \geq 2$ ) non-trivial elements and proceeded to extend this state from $\mathcal{W}_{m}$ to its "canonical" non- $M V$-Girard algebra extension, say $\mathcal{W}_{m}^{\mathcal{N}_{1}}$. The latter is defined as the set of all pairs $(a, b)$ of elements $a, b$ of $\mathcal{W}_{m}$ with $a \leq b$ equipped with a certain Girard algebra structure. Unfortunately, he proved the non-existence of additive state extensions on $\mathcal{W}_{m}^{\mathcal{N}_{1}}$ if $m$ exceeds the number 3 . In this unwelcome situation Weber decided that the condition used in (1) is too strong and looked for a weaker notion of an additivity. He proposed to define "weakly additive" states on a Girard algebra by the additivity only on its sub- $M V$-algebras. Then the condition used in (1) reduces to the usual property of the disjointness in $M V$-algebras. Worse, then the implied original idea of disjointness in Girard algebras has no longer meaning.

Meanwhile, Gylys (2010) used the antecedent of the implication (1) as a foundation of the following partial multiplicativity in a Girard algebra $\left(Q ; \wp, \wp^{-}\right)$(with $\wp$ viewed as a multiplication): a partial product $x \dot{\wp} y$ is defined in $Q$ and is equal to $x \wp y$ whenever the condition used in (1) is satisfied. Then he proposed the concept of effectible Girard algebras whose important characteristic consists in preserving the associativity when restricting their (total) multiplications to partial ones, which in contrast to the original multiplications become cancellative-consequently, we arrive at effect algebras. But we know from the theory of these algebras (see book by Dvurečenskij \& Pulmanova, 2000) that there is a well-defined concept of a state on them. Adapting this notion to a new circumstance we come to the following definition: a state on an effectible Girard algebra $(Q ; \wp, \wp-, T)$ (with $\top$ the top element 
of $Q)$ is a mapping st: $Q \rightarrow[0,1]$ such that $s t(\top)=1$, and $s t(x \dot{y} y)=s t(x)+s t(y)$, whenever the partial product $x \dot{y} y$ is defined in $Q$-it is nothing but the additive state in the sense of (1). It appeared that all $M V$-algebras are effectible and that principal non-trivial examples of effectible non- $M V$-Girard algebras are only mentioned canonical extensions $\mathcal{W}_{2}^{\mathcal{N}_{1}}$ and $\mathcal{W}_{3}^{\mathcal{N}_{1}}$ of $M V$-algebras $\mathcal{W}_{2}$ and $\mathcal{W}_{3}$, respectively. Gylys (2012) made an attempt to find more examples of effectible Girard algebras. For this reason, he was looking for finite and infinite families of elements of $\mathcal{W}_{m}$ instead of working with their traditional pairs. But the sight was really pitiful-he short-listed only the canonical extensions $\mathcal{W}_{2}^{\mathcal{N}_{1}}, \mathcal{W}_{3}^{\mathcal{N}_{1}}$, and 2-extensions $\mathcal{W}_{2}^{\mathcal{N}_{2}}, \mathcal{W}_{3}^{\mathcal{N}_{2}}$ of $M V$-chains $\mathcal{W}_{2}$ and $\mathcal{W}_{3}$, respectively. Additionally, an $n$-extension of $\mathcal{W}_{m}$ is the set of all the ordered $(n+1)$-tuples $\left\langle a_{0}, a_{1}, \ldots, a_{n}\right\rangle$ of elements $a_{0}, a_{1}, \ldots, a_{n}$ of $\mathcal{W}_{m}$ with $a \geq a_{1} \geq \ldots \geq a_{n}$ equipped with a certain Girard algebra structure. When building extensions, say $\tilde{s}$, of the unique states on $\mathcal{W}_{2}$ and $\mathcal{W}_{3}$ to those four effectible Girard algebras, the motor nerve is certainly the associativity of their partial multiplications. By applying $\tilde{s}$ to all possible partial binary products one can form and solve four complete and consistent systems of linear equations -this is a way to access to extended states on $\mathcal{W}_{2}^{\mathcal{N}_{1}}$, $\mathcal{W}_{3}^{\mathcal{N}_{1}}, \mathcal{W}_{2}^{\mathcal{N}_{2}}$ and to $\mathcal{W}_{3}^{\mathcal{N}_{2}}$. One may similarly toy with non-effectible Girard algebras $\mathcal{W}_{m}^{\mathcal{N}_{n}}$ with $n \geq 3, m \geq 2$ and $n \geq 1, m \geq 4$ and ascertain the truth about analogous systems of linear equations that they are never consistent.

In this very negative perspective, the present paper continues the author's recent investigations, for the first time in the case of non-effectible Girard algebras. We propose a new notion of states on these algebras. The only trouble with the definition is the existence of states in the mentioned problematic examples. In the paper we restrict ourselves to the examination of state extensions of the unique states on $M V$-chains $\mathcal{W}_{2}, \mathcal{W}_{3}, \mathcal{W}_{4}, \mathcal{W}_{5}$ and $\mathcal{W}_{6}$ to canonical extensions $\mathcal{W}_{4}^{\mathcal{N}_{1}}, \mathcal{W}_{5}^{\mathcal{N}_{1}}, \mathcal{W}_{6}^{\mathcal{N}_{1}}, 2$-extensions $\mathcal{W}_{4}^{\mathcal{N}_{2}}, \mathcal{W}_{5}^{\mathcal{N}_{2}}, 3$-extensions $\mathcal{W}_{2}^{\mathcal{N}_{3}}, \mathcal{W}_{3}^{\mathcal{N}_{3}}, \mathcal{W}_{4}^{\mathcal{N}_{3}}$, and also to 4-extensions $W_{2}^{N_{4}}, W_{3}^{N_{4}}$, respectively. We are faced with a somewhat technical difficulty in solving inconsistent systems of linear equations. But we surmount these obstacles - our method of rejection contradictory equations seem to be succeeding. Finally, we compare our states with Weber's weakly additive states and find our more "fit".

The paper is organized as follows. In Section 2 we recall the definitions of Girard algebras and $M V$-algebras, and present several important examples of such structures. In Section 3, we introduce our main concept of fit states on non-effectible Girard algebras. In this section a great many examples of such states on $n$-extensions of finite $M V$ chains are presented. Finally, in Appendix proofs of Theorem 11, Theorem 12, Theorem 13, Theorem 14, Theorem 15, Theorem 16 and Theorem 17 are presented. Fit states have partial additivity property, i.e., additivity on all "steady" and possibly on some "deflected" products. By the way, the author prefers to speak of multiplicativity property of states to be discussed on closer examination.

\section{A Girard Algebra of $n$-Chains in Weber $M V$-Chain}

Given a bounded lattice $(L ; \leq, \vee, \wedge, \top, \perp)$ under the partial ordering $\leq$, with binary join and meet operations $\vee, \wedge$ and with greatest element $T$ and least element $\perp$. A lattice-ordered commutative monoid $(L ; \leq, \otimes, T)$ has an associative commutative binary operation $\otimes$ that is order preserving in each argument and $\mathrm{T}$ is its unit element, i.e. $a \otimes \mathrm{T}=a$. A lattice-ordered commutative monoid is residuated if there is a binary operation $\otimes /$ (denoted below by $\otimes-$ once) on $L$, called the residual of $\otimes$, satisfying the condition $a \otimes b \leq c \Leftrightarrow a \leq c_{\otimes} / b$. The residual operation is monotone in its left (upper) arguments and antitone in its right (lower, respectively). A Girard algebra L is a residuated lattice-ordered commutative monoid equipped with the unary operation $\neg: L \rightarrow L$ (termed negation) defined by $\neg a:=\perp_{\otimes} / a$ provided that $\perp_{\otimes} /\left(\perp_{\otimes} / a\right)=a$; it is an involution: $\neg \neg a=a$. An MV-algebra is a Girard algebra $L$ satisfying $a_{\otimes} /\left(a_{\otimes} / b\right)=b_{\otimes} /\left(b_{\otimes} / a\right)$ for all $a, b \in Q$. A Boolean algebra is an $M V$-algebra satisfying $\otimes=\wedge$.

On most occasions, for Girard algebras and $M V$-algebras $L$, another commutative multiplication on $L$ (say $\wp$, such that $a \wp \perp=a$ for all $a \in L$ ) and another binary operation on $L$ (say $\wp /$ or $_{\wp}-$ ), called dual residual is used; they are related by

$$
\neg(a \otimes b)=\neg a \wp \neg b, \otimes \frac{a}{b}=a \wp \neg b, \wp_{\wp} \frac{a}{b}=a \otimes \neg b, \neg a={ }_{\wp} \frac{\top}{a} \text { and } \neg\left(\otimes \frac{a}{b}\right)={ }_{\wp} \frac{b}{a} .
$$

Moreover, $L$ is also dually residuated; it satisfies the following condition: $a \wp b \geq c \Leftrightarrow a \geq c_{\wp} / b$.

Usually, the authors denote the dual operation $\wp$ by some summation symbol such as $\oplus$ or simply + . But I prefer to view it as a second multiplication. For, turn to corresponding structures of (Girard's) linear logic (see research by Girard, 2004). This logic contains two idempotent "additives": the "positive" connective "Plus" (usually denoted by $\oplus$ ) and the "negative" connective "With" (denoted by \&), which can be modelled by lattice operations $\vee$ and $\wedge$, respectively. Moreover, it still contains two (not necessarily idempotent) "multiplicatives": the positive connective "Times" (denoted by $\otimes$ ) and the negative connective "Par" (denoted by reversed symbol to $\&$ ). The basic principle 
of linear logic is that the connectives of the same polarity commute, i.e., the identities:

$$
(a \oplus b) \otimes c \sim(a \otimes c) \oplus(b \otimes c) \text { and }(a \& b) \wp c \sim(a \wp c) \&(b \wp c)
$$

hold, where we used the Weierstrass $p$ to denote "Par"; this property corresponds to the distributivity of a Girard algebra. Thus, in view of the J.-M. Andreoli and J.-Y. Girard polarity (positive/negative) (see paper by Girard, 2004), the operation $\otimes$ is meant as a positive multiplication and the operation $\wp$ as a negative multiplication. Moreover, the join $\vee$ is used as a positive addition, and the meet $\wedge$ as a negative addition.

From now on, we deal with Girard algebras and $M V$-algebras in the absence of "positive" operations $\otimes$ and $\otimes /$, because they are not employed through the paper. We present several examples of $M V$-algebras and Girard algebras.

\section{Example 1}

(i) The real unit interval $[0,1]$ equipped with the multiplication $\wp$, the residual $\wp-$ and with the negation $\neg$ given by

$$
a \wp b=\min (a+b, 1), \wp \frac{c}{b}=\max (c-b, 0) \text { and } \neg a=1-a
$$

is an $M V$-algebra referred to as Łukasiewicz algebra.

(ii) For each $m=1,2, \ldots$, the set

$$
L_{m+1}:=\left\{0, \frac{1}{m}, \ldots, \frac{m-1}{m}, 1\right\}
$$

equipped with operations as in the Łukasiewicz algebra is an $M V$-algebra (also called the Łukasiewicz $M V$-chain).

(iii) The finite chain of integers $\mathcal{N}_{m}=\{0,1, \ldots, m\}$ equipped with the multiplication $\wp$, the residual $\wp-$ and the negation $\neg$ defined by

$$
i \wp j=\min (i+j, m),{ }_{\wp} \frac{k}{j}=\max (k-j, 0) \text { and } \neg k=m-k
$$

is an $M V$-algebra.

(iv) For $m \geq 2$, let $\mathcal{W}_{m}$ be the chain with exactly $m-1$ different non-trivial elements, say $\perp=$ : $a^{0}<a^{1}<\cdots<$ $a^{m-1}<a^{m}:=\mathrm{T}$. Then $\mathcal{W}_{m}$ has a unique $M V$-algebra structure given by

$$
a^{i} \wp a^{j}=a^{\min (i+j, m)}, \wp \frac{a^{k}}{a^{j}}=a^{\max (k-j, 0)} \text { and } \neg a^{k}=a^{m-k} .
$$

Furthermore follow:

$$
a^{k}=\underbrace{a^{1} \dot{\wp} \ldots \dot{\wp} a^{1}}_{k \text { times }} \text { and } a^{j+k}=a^{j} \dot{\wp} a^{k} \text { if } j+k \leq m,
$$

where $\dot{\wp}$ is the partially defined binary operation on $\mathcal{W}_{m}: a \dot{\wp} b$ is defined and equal to $a \wp b$ when $a \leq \neg b$. This $M V$-algebra first introduced by Weber (2010, Theorem 1.5) will be referred to as Weber $M V$-chain.

(v) For integers $m \geq 2$ and $n \geq 1$, let $W_{m}^{\mathcal{N}_{n}}$ be the class of order-reversing families $\left\langle a^{i_{0}}, a^{i_{1}}, \ldots, a^{i_{n}}\right\rangle$ (with $i_{0} \geq i_{1} \geq$ $\ldots \geq i_{n}$ ) of elements of Weber $M V$-chain $\mathcal{W}_{m}$ called $n$-chains. This class termed an $n$-extension of $\mathcal{W}_{m}$ (where $\mathcal{W}_{m}$ is identified with its diagonal $\left\{\left\langle a^{i_{0}}, a^{i_{0}}, \ldots, a^{i_{0}}\right\rangle \mid a^{i_{0}} \in \mathcal{W}_{m}\right\}$ has a unique non- $M V$-Girard algebra structure given by

$$
\begin{gathered}
\left\langle a^{i_{0}}, a^{i_{1}}, \ldots, a^{i_{n}}\right\rangle \leq\left\langle a^{j_{0}}, a^{j_{1}}, \ldots, a^{i_{n}}\right\rangle \Leftrightarrow i_{0} \leq j_{0}, i_{1} \leq j_{1}, \ldots, i_{n} \leq j_{n}, \\
\left\langle a^{i_{0}}, a^{i_{1}}, \ldots, a^{i_{n}}\right\rangle \wp\left\langle a^{j_{0}}, a^{j_{1}}, \ldots, a^{j_{n}}\right\rangle=\left\langle a^{\min \left(i_{0}+j_{0}, m\right)}, a^{\min \left(i_{1}+j_{0}, i_{0}+j_{1}, m\right)}, \ldots, a^{\min \left(i_{n}+j_{0}, i_{n-1}+j_{1}, \ldots i_{0}+j_{n}, m\right)}\right\rangle, \\
\wp \frac{\left\langle a^{k_{0}}, a^{k_{1}}, \ldots, a^{k_{n}}\right\rangle}{\left\langle a^{j_{0}}, a^{j_{1}}, \ldots, a^{j_{n}}\right\rangle}=\left\langle a^{\max \left(k_{0}-j_{0}, k_{1}-j_{1}, \ldots, k_{n}-j_{n}, 0\right)}, a^{\max \left(k_{1}-j_{0}, \ldots, k_{n}-j_{n-1}, 0\right)}, \ldots, a^{\max \left(k_{n}-j_{0}, 0\right)}\right\rangle
\end{gathered}
$$

and

$$
\neg\left\langle a^{i_{0}}, a^{i_{1}}, \ldots, a^{i_{n}}\right\rangle=\left\langle a^{m-i_{n}}, a^{m-i_{n-1}}, \ldots, a^{m-i_{0}}\right\rangle .
$$

Note that $n$-extensions of $M V$-algebras in the case of $n=1$ were first proposed in research by Höhle and Weber (1997), and in the case of an arbitrary integer $n$ in paper by Gylys (2012).

Let $(L ; \wp, \neg)$ be an $M V$-algebra. Recall that a state on $L$ is any mapping $s t: L \rightarrow[0,1]$ such that (i) $s t(T)=1$, and (ii) $s t(a \dot{\wp} b)=s t(a)+s t(b)$ whenever $a \dot{\wp} b$ is defined in $L$. Additionally, the partial products $a \dot{\wp} b$ exist in $L$ and are 
equal to $a \wp b$ when $a \leq \neg b$. Usually, the authors say that a state has the additivity property. But I prefer to drop this term and to speak of multiplicativity property of states on $M V$-algebras or on Girard algebras.

\section{Fit States on $n$-Extensions of Weber $M V$-Chains}

Now, we are almost ready to introduce our main concept of fit states on Girard algebras. Let us still recall several definitions and facts.

Definition 2 (Gylys, 2010, Definition 3.5) A Girard algebra $(Q ; \wp)$ is said to be effectible when its partial multiplication $\dot{\wp}$ satisfies associativity: the implication $\exists x \dot{\wp} y, \exists(x \dot{\varphi} y) \dot{\wp} z \Rightarrow \exists y \dot{\wp} z, \exists x \dot{\wp}(y \dot{\wp} z)$ holds for all $x, y, z \in Q$. Additionally, the partial products $x \dot{\wp} y$ exist in $Q$ when the following restrictions hold (introduced by Höhle \& Weber, 1997):

$$
\wp \frac{x \wp y}{x}=y \text { and } \wp \frac{x \wp y}{y}=x .
$$

Definition 3 (Gylys, 2012, Definition 6.2) Let $(Q ; \wp)$ be an effectible Girard algebra. A state on $Q$ is any mapping st: $L \rightarrow[0,1]$ such that (i) $s t(T)=1$, and (ii) $s t(x \dot{y} y)=s t(x)+\operatorname{st}(y)$ whenever $x \dot{\wp} y$ is defined in $Q$.

In fact, this concept of a state is well known-at least for effect algebras (see Dvurečenskij \& Pulmanova, 2000, p. 30)-but effectible Girard algebras with multiplications restricted to partial ones are effect algebras as well (Gylys, 2010, Proposition 3.11).

All $M V$-algebras (and therefore Weber $M V$-chains $\mathcal{W}_{m}$ ) are examples of effectible Girard algebras. But the list of known effectible non- $M V$-algebras is short: 1-extensions $\mathcal{W}_{2}^{\mathcal{N}_{1}}, \mathcal{W}_{3}^{\mathcal{N}_{1}}$ and 2-extensions $\mathcal{W}_{2}^{\mathcal{N}_{2}}, \mathcal{W}_{3}^{\mathcal{N}_{2}}$ of Weber $M V$-chains $\mathcal{W}_{2}$ and $\mathcal{W}_{3}$, respectively. (Such algebra is also the real unit interval [0,1] equipped with the "nilpotent maximum" $t$-conorm (introduced by Fodor, in 1995).

Proposition 4 (Weber, 2009, Corollary 5.2) For any natural number $m$, the Weber MV-chain $W_{m}$ has a unique state s given by

$$
s\left(a^{k}\right)=\frac{k}{m} \text { for } k=0,1, \ldots, m .
$$

(Therefore, s can also be seen as an isomorphism from $\mathcal{W}_{m}$ onto the Łukasiewicz $M V$-chain $L_{m+1}$.)

Now we start with the unique state $s$ on $\mathcal{W}_{m}$ (with some natural number $m$ ) and look for state extensions $\tilde{s}$ on $\mathcal{W}_{m}^{\mathcal{N}_{n}}$. For the mentioned cases $m=2,3$ and $n=1,2$, positive results are obtained by Weber and the author. Unfortunately, for $m \geq 4$ and $n \geq 1$ and for $m \geq 2$ and $n \geq 3$, n-extensions of Weber $M V$-chains are not effectible, and it is not clear what "state" $\tilde{s}$ extends $s$. From now on, elements $a^{0}, a^{1}, \ldots, a^{m}$ of the Weber $M V$-chain $\mathcal{W}_{m}$ will be simply denoted by $0,1, \ldots, m$, and $n$-chains $\left\langle a^{i_{0}}, a^{i_{1}}, \ldots, a^{i_{n}}\right\rangle$ (with $i_{0} \geq i_{1} \geq \ldots \geq i_{n}$ ) of $\mathcal{W}_{m}^{\mathcal{N}_{n}}$ will be denoted by $i_{0} i_{1} \ldots i_{n}$, for short.

Theorem 5 The Weber $M V$-chains $\mathcal{W}_{m}$ for $m=2,3$ permit states on their $n$-extensions $\mathcal{W}_{m}^{\mathcal{N}_{n}}$ of $\mathcal{W}_{m}$ with $n=1,2$.

(i) For $m=2$ and $n=1$ (Weber, 2009, Example 3.5(a)): The unique state $s$ on $\mathcal{W}_{2}$, given by $s_{0}=0, s_{1}=1 / 2$ and $s_{2}=1$, has a unique state extension $\tilde{s}$ on $\mathcal{W}_{2}^{\mathcal{N}_{1}}$ defined by $\tilde{s}_{00}=0, \tilde{s}_{10}=1 / 3, \tilde{s}_{11}=1 / 2, \tilde{s}_{20}=1 / 2, \tilde{s}_{21}=2 / 3$, and $\tilde{s}_{22}=1$.

(ii) For $m=3$ and $n=1$ (Weber, 2009, Example 3.5(b)): The unique state $s$ on $W_{3}$, given by $s_{0}=0, s_{1}=1 / 3$, $s_{2}=2 / 3$ and $s_{3}=1$, has a unique state extension $\tilde{s}$ on $W_{3}^{\mathcal{N}_{1}}$ defined by $\tilde{s}_{00}=0, \tilde{s}_{10}=1 / 4, \tilde{s}_{11}=1 / 3, \tilde{s}_{20}=3 / 8$, $\tilde{s}_{21}=1 / 2, \tilde{s}_{30}=1 / 2, \tilde{s}_{31}=5 / 8, \tilde{s}_{32}=3 / 4$, and $\tilde{s}_{33}=1$.

(iii) For $m=2$ and $n=2$ (Gylys, 2012, Theorem 6.10(iii)): The unique state $s$ on $\mathcal{W}_{2}$ has a unique state extension $\tilde{s}$ on $W_{2}^{\mathcal{N}_{2}}$ given by $\tilde{s}_{000}=0, \tilde{s}_{100}=1 / 4, \tilde{s}_{110}=3 / 8, \tilde{s}_{111}=1 / 2, \tilde{s}_{200}=1 / 3, \tilde{s}_{210}=1 / 2, \tilde{s}_{211}=5 / 8, \tilde{s}_{220}=2 / 3$, $\tilde{s}_{221}=3 / 4$, and $\tilde{s}_{222}=1$.

(iv) For $m=3$ and $n=2$ (Gylys, 2012, Theorem 6.10(iv)): The unique state $s$ on $\mathcal{W}_{3}$ has a unique state extension $\tilde{s}$ on $W_{3}^{\mathcal{N}_{2}}$ given by $\tilde{s}_{000}=0, \tilde{s}_{100}=1 / 5, \tilde{s}_{110}=4 / 15, \tilde{s}_{111}=1 / 3, \tilde{s}_{200}=4 / 15, \tilde{s}_{210}=2 / 5, \tilde{s}_{211}=7 / 15, \tilde{s}_{220}=7 / 15$, $\tilde{s}_{221}=8 / 15, \tilde{s}_{222}=2 / 3, \tilde{s}_{300}=1 / 3, \tilde{s}_{310}=7 / 15, \tilde{s}_{311}=8 / 15, \tilde{s}_{320}=8 / 15, \tilde{s}_{321}=3 / 5, \tilde{s}_{322}=11 / 15, \tilde{s}_{330}=2 / 3$, $\tilde{s}_{331}=11 / 15, \tilde{s}_{332}=4 / 5$, and $\tilde{s}_{333}=1$.

Now we are preparing for a study of state extensions $\tilde{s}$ on non-effectible Girard algebras $\mathcal{W}_{m}^{\mathcal{N}_{n}}$ for $m \geq 4, n \geq 1$ and for $m \geq 2, n \geq 3$.

Let $(Q ; \dot{\ominus})$ be a non-effectible Girard algebra equipped with the partial multiplication $\dot{\wp}$. Consider the following "non-associative" situations: 
(1) The partial triple product $(a \dot{\wp} b) \dot{\wp} c \neq T$ is defined (i.e. the partial binary products involved are defined) for some elements $a, b, c \in L$, but neither $a \dot{\wp}(b \dot{\wp} c)$ nor $b \dot{\wp}(a \dot{\wp} c)$ are not;

(2) The partial triple products $(a \dot{\wp} b) \dot{\wp} c \neq \mathrm{T}$ and $a \dot{\wp}(b \dot{\wp} c) \neq \mathrm{T}$ are both defined, but $b \dot{\wp}(a \dot{\wp} c)$ is not;

(3) The partial triple products $(a \dot{\wp} b) \dot{\wp} c \neq \mathrm{T}$ and $b \dot{\wp}(a \dot{\wp} c) \neq \mathrm{T}$ are both defined, but $a \dot{\wp}(b \dot{\wp} c)$ is not.

If the situation (1) takes place then we say that the partial binary products $a \dot{\wp} b(=a \wp b)$ and $(a \wp b) \dot{\wp} c(=a \wp b \wp c)$ are deflected. If the situation (2) occurs then the partial binary products $a \dot{\wp} b(=a \wp b),(a \wp b) \dot{\wp} c(=a \wp b \wp c), b \dot{\wp} c(=b \wp c)$ and $a \dot{\wp}(b \wp c)(=a \wp b \wp c)$ are deflected, while in the situation (3) the following partial binary products: $a \dot{\wp} b(=a \wp b)$, $(a \wp b) \dot{\wp} c(=a \wp b \wp c), a \wp c(=a \wp c)$ and $b \dot{\wp}(a \wp c)(=a \wp b \wp c)$ are deflected. A partial binary product $a \dot{\wp} b=a \wp b$ is steady when it is not deflected. It is clear that partial products $a \dot{\wp} \neg a=\mathrm{T}$ are always steady. Moreover, at all times partial products within an effectible Girard algebra are steady.

Definition 6 Let $(Q ; \dot{\wp})$ be a non-effectible Girard algebra equipped with the partial multiplication $\dot{\wp}$.

(1) A fit state on $Q$ is any mapping st: $Q \rightarrow[0,1]$ such that

(i) $s t(\mathrm{~T})=1$ (boundary condition);

(ii) $x \leq y \Rightarrow s t(x) \leq s t(y)$ (isotonicity);

(iii) the equality $s t(x \dot{\wp} y)=s t(x)+s t(y)$ (partial multiplicativity or PM, for short) holds on all steady and possibly on some deflected binary partial products $x \dot{\wp} y$ within $Q$.

(2) Weber state on $Q$ is an isotonic map st: $Q \rightarrow[0,1]$ satisfying the boundary condition and fulfilling PM only on all $M V$-subalgebras of $Q$ (see Weber, 2009, 2010).

Let us put the results from research by Weber (2010) concerning "weakly additive" extensions of the unique state $s$ on an $M V$-chain $\mathcal{W}_{m}$ to its canonical extension (1-extension in our terminology) $\mathcal{W}_{m}^{N_{1}}$. In paper by Weber (2010, Theorems 2.5 and 2.6), it is proved that all $M V$-subalgebras of $\mathcal{W}_{m}^{N_{1}}$ can be identified as Weber $M V$-chains. Moreover, Weber established the following fact.

Theorem 7 (Weber, 2010, Corollary 3.2) There exists a unique weakly additive measure (Weber state in our terminology) $W$ on $\mathcal{W}_{m}^{\mathcal{N}_{1}}$ given by $W_{k j}=k /(m+k-j)$ for all $k \geq j$.

This formula can be written in the form $W_{k j}=M\left(s_{k}, s_{j}\right)$, based on the special "mean value function" $M(x, y)=$ $x /(1+x-y)$.

Unfortunately, Weber's works do not deal with $n$-extensions $\mathcal{W}_{m}^{\mathcal{N}_{n}}$ with $n>1$.

Concerning non-effectible Girard algebras $\mathcal{W}_{m}^{\mathcal{N}_{n}}$ for $m \geq 4, n \geq 1$ and for $m \geq 2, n \geq 3$, let us denote by $E_{m}^{n}$, $S_{m}^{n}$ and $D_{m}^{n}$ the set of all possible existing partial binary products within $\mathcal{W}_{m}^{\mathcal{N}_{n}}$, the set of all existing steady partial binary products within $\mathcal{W}_{m}^{\mathcal{N}_{n}}$, and the set of all existing deflected partial binary products within $\mathcal{W}_{m}^{\mathcal{N}_{n}}$, respectively.

Theorem 8 The unique state $s$ on $M V$-chain $\mathcal{W}_{4}$ defined by $s_{0}=0, s_{1}=1 / 4, s_{2}=1 / 2, s_{3}=3 / 4$ and $s_{4}=1$, has a unique fit state extension $\tilde{s}$ on $W_{4}^{N_{1}}$ defined by

$\tilde{s}_{00}=0, \tilde{s}_{10}=1 / 5, \tilde{s}_{11}=1 / 4, \tilde{s}_{20}=1 / 3, \tilde{s}_{21}=2 / 5, \tilde{s}_{22}=1 / 2, \tilde{s}_{30}=2 / 5, \tilde{s}_{31}=1 / 2, \tilde{s}_{32}=3 / 5, \tilde{s}_{33}=3 / 4$, $\tilde{s}_{40}=1 / 2, \tilde{s}_{41}=3 / 5, \tilde{s}_{42}=2 / 3, \tilde{s}_{43}=4 / 5$, and $\tilde{s}_{44}=1$.

Proof. We have that $W_{4}^{N_{1}}$ consists of 15 elements: $00<10<11,20<21<22,30<31<32<33$, $40<41<42<43<44$. The set $E_{4}^{1}$ of all possible existing partial products is the following:

$10 \dot{\wp} 10=21,10 \dot{\wp} 20=31,10 \dot{\wp} 21=32,10 \dot{\wp} 30=41,10 \dot{\wp} 31=42,10 \dot{\wp} 32=43,10 \dot{\wp} 43=44,11 \dot{\wp} 11=22$, $11 \dot{\wp} 22=33,11 \dot{\wp} 33=44,20 \dot{\wp} 20=42,20 \dot{\wp} 31=43,20 \dot{\wp} 42=44,21 \dot{\wp} 21=43,21 \dot{\wp} 32=44,22 \dot{\wp} 22=44$, $30 \dot{\wp} 30=43,30 \dot{\wp} 41=44,31 \dot{\wp} 31=44,40 \dot{\wp} 40=44$ and $x \dot{\wp} 00=x$ for all $x \in \mathcal{W}_{4}^{N_{1}}$.

Using this list we verify the existence of all possible partial triple products. We find two "non-associative" situations:

$$
\underbrace{(10 \dot{\wp} 20)}_{=31} \dot{\wp} 10=42 \text { is defined but } 20 \dot{\wp} \underbrace{(10 \dot{\wp} 10)}_{=21} \text { is not }
$$

and

$$
\underbrace{(10 \dot{\varnothing} 20)}_{=31} \dot{\wp} 20=43 \text { is defined but } 10 \dot{\wp} \underbrace{(20 \dot{\wp} 20)}_{=42} \text { is not. }
$$

Thus, the following partial binary products $10 \dot{\wp} 20=31,10 \dot{\wp} 31=42$ and $20 \dot{\wp} 31=43$ are deflected, while steady 
partial binary products are as follows:

$10 \dot{\wp} 10=21,10 \dot{\wp} 21=32,10 \dot{\wp} 30=41,10 \dot{\wp} 32=43,10 \dot{\wp} 43=44,11 \dot{\wp} 11=22,11 \dot{\wp} 22=33,11 \dot{\wp} 33=44$, $20 \dot{\wp} 20=42,20 \dot{\wp} 42=44,21 \dot{\wp} 21=43,21 \dot{\wp} 32=44,22 \dot{\wp} 22=44,30 \dot{\wp} 30=43,30 \dot{\wp} 41=44,31 \dot{\wp} 31=44$ and $40 \dot{\wp} 40=44$.

Applying $\tilde{s}$ to all steady partial binary products within $\mathcal{W}_{4}^{\mathcal{N}_{1}}$, we obtain the following system of linear equations:

$2 \tilde{s}_{10}=\tilde{s}_{21}, \tilde{s}_{10}+\tilde{s}_{21}=\tilde{s}_{32}, \tilde{s}_{10}+\tilde{s}_{30}=\tilde{s}_{41}, \tilde{s}_{10}+\tilde{s}_{32}=\tilde{s}_{43}, \tilde{s}_{10}+\tilde{s}_{43}=\tilde{s}_{44}, 2 \tilde{s}_{11}=\tilde{s}_{22}, \tilde{s}_{11}+\tilde{s}_{22}=\tilde{s}_{33}, \tilde{s}_{11}+\tilde{s}_{33}=\tilde{s}_{44}$, $2 \tilde{s}_{20}=\tilde{s}_{42}, \tilde{s}_{20}+\tilde{s}_{42}=\tilde{s}_{44}, 2 \tilde{s}_{21}=\tilde{s}_{43}, \tilde{s}_{21}+\tilde{s}_{32}=\tilde{s}_{44}, 2 \tilde{s}_{22}=\tilde{s}_{44}, 2 \tilde{s}_{30}=\tilde{s}_{43}, \tilde{s}_{30}+\tilde{s}_{41}=\tilde{s}_{44}, 2 \tilde{s}_{31}=\tilde{s}_{44}, 2 \tilde{s}_{40}=\tilde{s}_{44}$.

In view of the extension property: $\tilde{s}_{00}=s_{0}=0, \tilde{s}_{11}=s_{1}=1 / 4, \tilde{s}_{22}=s_{2}=1 / 2, \tilde{s}_{33}=s_{3}=3 / 4$ and $\tilde{s}_{44}=s_{4}=1$, we solve this system and obtain the solution, as states.

Observe that 1-extension $\mathcal{W}_{4}^{\mathcal{N}_{1}}$ of the Weber $M V$-chain $\mathcal{W}_{4}$ contains the following five Weber $M V$-chains:

$00<10<10^{2 \dot{\varphi}}(=21)<10^{3 \dot{\varphi}}(=32)<10^{4 \dot{\varphi}}(=43)<10^{5 \dot{\varphi}}(=44), 00<11<11^{2 \dot{\varphi}}(=22)<11^{3 \dot{\varphi}}(=33)<11^{4 \dot{\varphi}}(=$ 44), $00<20<20^{2 \dot{\phi}}(=42)<20^{3 \dot{\phi}}(=44), 00<31<31^{2 \dot{\phi}}(=44)$ and $00<40<40^{2 \dot{\phi}}(=44)$.

It can be seen from Theorem 7 and the preceding proposition that values of the fit state $\tilde{s}$ on $\mathcal{W}_{4}^{\mathcal{N}_{1}}$ (except values $\tilde{s}_{30}$ and $\tilde{s}_{41}$ ) are in line with those of Weber state $W$. By definition of $W$, we have that it fulfils PM only on all partial binary products in enumerated $M V$-chains, i.e. on

$10 \dot{\wp} 10=21,10 \dot{\wp} 21=32,10 \dot{\wp} 32=43,10 \dot{\wp} 43=44,21 \dot{\wp} 21=43,21 \dot{\wp} 32=44,11 \dot{\wp} 11=22,11 \dot{\wp} 22=33$, $11 \dot{\wp} 33=44,22 \dot{\wp} 22=44,20 \dot{\wp} 20=42,20 \dot{\wp} 42=44,31 \dot{\wp} 31=44$ and on $40 \dot{\wp} 40=44$;

by the preceding theorem, everyone is steady product within $\mathcal{W}_{4}^{\mathcal{N}_{1}}$. But this list of steady products is not full - the remainder is the following: $10 \dot{\wp} 30=41,30 \dot{\wp} 30=43$ and $30 \dot{\wp} 41=44$. Thus, the state $\tilde{s}$ on $W_{4}^{\mathcal{N}_{1}}$ fulfils PM on a little larger family of partial binary products than Weber state $W$ does.

Theorem 9 The unique state $s$ on $\mathcal{W}_{5}$, given by $s_{0}=0, s_{1}=1 / 5, s_{2}=2 / 5, s_{3}=3 / 5, s_{4}=4 / 5$ and $s_{5}=1$, has an infinite family of fit state extensions $\tilde{s}$ on $\mathcal{W}_{5}^{\mathcal{N}_{1}}$ defined by

$\tilde{s}_{00}=0, \tilde{s}_{10}=1 / 6, \tilde{s}_{11}=1 / 5, \tilde{s}_{20}=1-2 p, \tilde{s}_{21}=1 / 3, \tilde{s}_{22}=2 / 5, \tilde{s}_{30}=p, \tilde{s}_{31}=4 p-1, \tilde{s}_{32}=1 / 2, \tilde{s}_{33}=3 / 5$, $\tilde{s}_{40}=5 / 12, \tilde{s}_{41}=1 / 2, \tilde{s}_{42}=2-4 p, \tilde{s}_{43}=2 / 3, \tilde{s}_{44}=4 / 5, \tilde{s}_{50}=1 / 2, \tilde{s}_{51}=7 / 12, \tilde{s}_{52}=1-p, \tilde{s}_{53}=2 p, \tilde{s}_{54}=5 / 6$, and $\tilde{s}_{55}=1$,

where the parameter $p$ is located in the interval [1/3,3/8]. Among these fit state extensions there are three ones fulfilling PM not only on all steady binary products but also on several deflected binary products:

(1) if $p=1 / 3$, then in addition $\tilde{s}$ fulfils $P M$ on $10 \dot{\wp} 30=41,10 \dot{\wp} 41=52$ and $30 \dot{\wp} 41=54$;

(2) if $p=17 / 48$, then $\tilde{s}$ also satisfies PM on $10 \dot{\wp} 31=42$ and $31 \dot{\wp} 31=54$;

(3) if $p=13 / 36$, then $\tilde{s}$ accomplishes PM on $10 \dot{\wp} 20=31,10 \dot{\wp} 30=41,10 \dot{\wp} 42=53$ and $20 \dot{\wp} 42=54$.

Proof. We have that $W_{5}^{N_{1}}$ consists of 21 elements: $00<10<11,20<21<22,30<31<32<33$, $40<41<42<43<44,50<51<52<53<54<55$. A routine calculation shows that the set $E_{5}^{1}$ of all possible existing partial products is the following:

$10 \dot{\wp} 10=21,10 \dot{\wp} 20=31,10 \dot{\wp} 21=32,10 \dot{\wp} 30=41,10 \dot{\wp} 31=42,10 \dot{\wp} 32=43,10 \dot{\wp} 40=51,10 \dot{8} 41=52$, $10 \dot{\wp} 42=53,10 \dot{\wp} 43=54,10 \dot{\wp} 54=55,11 \dot{\wp} 11=22,11 \dot{\wp} 22=33,11 \dot{\wp} 33=44,11 \dot{\wp} 44=55,20 \dot{\wp} 20=42$, $20 \dot{\wp} 30=52,20 \dot{\wp} 31=53,20 \dot{\wp} 42=54,20 \dot{\wp} 53=55,21 \dot{\wp} 21=43,21 \dot{\wp} 32=54,21 \dot{\wp} 43=55,22 \dot{\varnothing} 22=44$, $22 \dot{\wp} 33=55,30 \dot{\wp} 30=53,30 \dot{\wp} 41=54,30 \dot{\wp} 52=55,31 \dot{\wp} 31=54,31 \dot{\wp} 42=55,32 \dot{\wp} 32=55,40 \dot{\wp} 40=54$, $40 \dot{\ominus} 51=55,41 \dot{\wp} 41=55,50 \dot{\wp} 50=55$ and $x \dot{\wp} 00=x$ for all $x \in \mathcal{W}_{5}^{N_{1}}$.

Now verifying each of the partial triple products in turn, we establish the following six exclusive situations:

$$
\begin{gathered}
\underbrace{(10 \dot{\wp} 20)}_{=31} \dot{\wp} 10=42 \text { is defined but } 20 \dot{\wp} \underbrace{(10 \dot{\wp} 10)}_{=21} \text { is not; } \\
\underbrace{(10 \dot{\wp} 20)}_{=31} \dot{\wp} 31=20 \dot{\wp} \underbrace{(10 \dot{\wp} 31)}_{=42}=54 \text { are defined but } 10 \dot{\wp} \underbrace{(20 \dot{\wp} 31)}_{=53} \text { is not; } \\
\underbrace{(10 \dot{\wp} 30)}_{=41} \dot{\wp} 10=52 \text { is defined but } 30 \dot{\wp} \underbrace{(10 \dot{\wp} 10)}_{=21} \text { is not; }
\end{gathered}
$$




$$
\begin{gathered}
\underbrace{(10 \dot{\wp} 30)}_{=41} \dot{\wp} 30=54 \text { is defined but } 10 \dot{\wp} \underbrace{(30 \dot{\wp} 30)}_{=53} \text { is not; } \\
\underbrace{(10 \dot{\wp} 31)}_{=42} \dot{\wp} 10=53 \text { is defined but } 31 \dot{\wp} \underbrace{(10 \dot{\ominus} 10)}_{=21} \text { is not; } \\
\underbrace{(10 \dot{\wp} 31)}_{=42} \dot{\wp} 20=31 \dot{\wp} \underbrace{(10 \dot{\wp} 20)}_{=31}=54 \text { are defined but } 10 \dot{\wp} \underbrace{(31 \dot{\wp} 20)}_{=53} \text { is not. }
\end{gathered}
$$

Thus, the set $D_{5}^{1}$ of deflected partial binary products within $W_{5}^{\mathcal{N}_{1}}$ is as follows: $10 \dot{\wp} 20=31,10 \dot{\wp} 30=41$, $10 \dot{\wp} 31=42,10 \dot{\wp} 41=52,10 \wp 42=53,20 \dot{\wp} 42=54,30 \dot{\wp} 41=54$ and $31 \dot{\wp} 31=54$. Now applying $\tilde{s}$ to all steady partial products within $\mathcal{W}_{5}^{N_{1}}$, we obtain the following system of linear equations:

$2 \tilde{s}_{10}=\tilde{s}_{21}, \tilde{s}_{10}+\tilde{s}_{21}=\tilde{s}_{32}, \tilde{s}_{10}+\tilde{s}_{32}=\tilde{s}_{43}, \tilde{s}_{10}+\tilde{s}_{40}=\tilde{s}_{51}, \tilde{s}_{10}+\tilde{s}_{43}=\tilde{s}_{54}, \tilde{s}_{10}+\tilde{s}_{54}=\tilde{s}_{55}, 2 \tilde{s}_{11}=\tilde{s}_{22}, \tilde{s}_{11}+\tilde{s}_{22}=\tilde{s}_{33}$, $\tilde{s}_{11}+\tilde{s}_{33}=\tilde{s}_{44}, \tilde{s}_{11}+\tilde{s}_{44}=\tilde{s}_{55}, 2 \tilde{s}_{20}=\tilde{s}_{42}, \tilde{s}_{20}+\tilde{s}_{30}=\tilde{s}_{52}, \tilde{s}_{20}+\tilde{s}_{31}=\tilde{s}_{53}, \tilde{s}_{20}+\tilde{s}_{53}=\tilde{s}_{55}, 2 \tilde{s}_{21}=\tilde{s}_{43}, \tilde{s}_{21}+\tilde{s}_{32}=\tilde{s}_{54}$, $\tilde{s}_{21}+\tilde{s}_{43}=\tilde{s}_{55}, 2 \tilde{s}_{22}=\tilde{s}_{44}, \tilde{s}_{22}+\tilde{s}_{33}=\tilde{s}_{55}, 2 \tilde{s}_{30}=\tilde{s}_{53}, \tilde{s}_{30}+\tilde{s}_{52}=\tilde{s}_{55}, \tilde{s}_{31}+\tilde{s}_{42}=\tilde{s}_{55}, 2 \tilde{s}_{32}=\tilde{s}_{55}, 2 \tilde{s}_{40}=\tilde{s}_{54}$, $\tilde{s}_{40}+\tilde{s}_{51}=\tilde{s}_{55}, 2 \tilde{s}_{41}=\tilde{s}_{55}$ and $2 \tilde{s}_{50}=\tilde{s}_{55}$.

We have that $\tilde{s}_{00}=s_{0}=0, \tilde{s}_{11}=s_{1}=1 / 5, \tilde{s}_{22}=s_{2}=2 / 5, \tilde{s}_{33}=s_{3}=3 / 5, \tilde{s}_{44}=s_{4}=4 / 5$ and $\tilde{s}_{55}=s_{5}=1$. In view of this extension property, the analysis of the system shows that it is not complete with $\tilde{s}_{30}$ as a parameter varying in the interval $[1 / 3,3 / 8]$ (by the isotonicity of $\tilde{s}$ ). To supplement the system, we are looking for consistent equations by applying $\tilde{s}$ to suitable deflected partial products. We discover that

(1) the system of linear equations together with the equation $\tilde{s}_{10}+\tilde{s}_{30}=\tilde{s}_{41}$;

(2) the system together with the equation $\tilde{s}_{10}+\tilde{s}_{31}=\tilde{s}_{42}$;

(3) the system together with the equation $\tilde{s}_{10}+\tilde{s}_{20}=\tilde{s}_{31}$

form three complete systems of linear equations yielding the required solutions. Finally, in these three cases applying $\tilde{s}$ to all deflected partial products, we complete the proof.

Observe that the 1-extension $\mathcal{W}_{5}^{\mathcal{N}_{1}}$ of the Weber $M V$-chain $\mathcal{W}_{5}$ contains the following four Weber $M V$-chains:

$00<10<10^{2 \dot{\varphi}}(=21)<10^{3 \dot{\varphi}}(=32)<10^{4 \dot{\varphi}}(=43)<10^{5 \dot{\varphi}}(=54)<10^{6 \dot{\varphi}}(=55), 00<11<11^{2 \dot{\varphi}}(=22)<11^{3 \dot{\varphi}}(=$ $33)<11^{4 \dot{\varphi}}(=44)<11^{5 \dot{\varphi}}(=55), 00<41<41^{2 \dot{\varphi}}(=55)$ and $00<50<50^{2 \dot{\varphi}}(=55)$.

From Theorem 7 and the preceding proposition it follows that values of all fit states $\tilde{s}$ on $\mathcal{W}_{5}^{\mathcal{N}_{1}}$ (except values $\tilde{s}_{20}$, $\tilde{s}_{30}, \tilde{s}_{31}, \tilde{s}_{40}, \tilde{s}_{42}, \tilde{s}_{51}, \tilde{s}_{52}$ and $\tilde{s}_{53}$ ) coincide with those of Weber state $W$. By definition of $W$, we have that it fulfils PM only on the next partial binary products:

$10 \dot{\wp} 10=21,10 \dot{\wp} 21=32,10 \dot{\wp} 32=43,10 \dot{\wp} 43=54,10 \dot{\wp} 54=55,21 \dot{\wp} 21=43,21 \dot{\wp} 32=54,32 \dot{\wp} 32=55$, $11 \dot{\wp} 11=22,11 \dot{\wp} 22=33,11 \dot{\wp} 33=44,11 \dot{\wp} 44=55,22 \dot{\wp} 22=44,22 \dot{\wp} 33=55,41 \dot{\wp} 41=55$ and $50 \dot{\wp} 50=55$;

by the preceding theorem, everyone is steady product within $W_{5}^{\mathcal{N}_{1}}$. But PM of $W$ cases to exist on all deflected products and on the next steady products:

$10 \dot{\wp} 40=51,20 \dot{\wp} 20=42,20 \dot{\wp} 30=52,20 \dot{\wp} 31=53,20 \dot{\wp} 53=55,30 \dot{\wp} 30=53,30 \dot{\wp} 52=55,31 \dot{\wp} 42=55$, $40 \dot{\wp} 40=54$ and $40 \dot{\wp} 51=55$.

Thus, at first sight, all fit states on $W_{5}^{\mathcal{N}_{1}}$ have a lead over Weber state $W$.

Theorem 10 The unique state $s$ on $\mathcal{W}_{6}$, given by $s_{0}=0, s_{1}=1 / 6, s_{2}=1 / 3, s_{3}=1 / 2, s_{4}=2 / 3, s_{5}=5 / 6$ and $s_{6}=1$, has an infinite family of fit state extensions $\tilde{s}$ on $W_{6}^{\mathcal{N}_{1}}$ defined by

$\tilde{s}_{00}=0, \tilde{s}_{10}=1 / 7, \tilde{s}_{11}=1 / 6, \tilde{s}_{20}=1 / 4, \tilde{s}_{21}=2 / 7, \tilde{s}_{22}=1 / 3, \tilde{s}_{30}=q / 2, \quad \tilde{s}_{31}=3 / 8, \tilde{s}_{32}=3 / 7, \tilde{s}_{33}=1 / 2$, $\tilde{s}_{40}=3 / 8, \tilde{s}_{41}=1-p, \tilde{s}_{42}=1 / 2, \tilde{s}_{43}=4 / 7, \tilde{s}_{44}=2 / 3, \tilde{s}_{50}=3 / 7, \tilde{s}_{51}=1 / 2, \tilde{s}_{52}=p, \tilde{s}_{53}=5 / 8, \tilde{s}_{54}=5 / 7$, $\tilde{s}_{55}=5 / 6, \tilde{s}_{60}=1 / 2, \tilde{s}_{61}=4 / 7, \tilde{s}_{62}=5 / 8, \tilde{s}_{63}=q, \tilde{s}_{64}=3 / 4, \tilde{s}_{65}=6 / 7$ and $\tilde{s}_{66}=1$,

indexed by $p$ and $q$ such that $1 / 2 \leq p \leq 5 / 8 \leq q \leq 3 / 4$. Among these fit state extensions there are six ones fulfilling PM not only on all steady partial binary products but also on some deflected partial binary products:

(1) if $p=11 / 21$ and $q=2 / 3$, then in addition $\tilde{s}$ fulfils $P M$ on $10 \dot{\wp} 30=41,10 \dot{\wp} 52=63$ and $30 \dot{\wp} 52=65$;

(2) if $p=31 / 56$ and $q=39 / 56$, then $\tilde{s}$ also satisfies $P M$ on $10 \dot{\wp 52}=63$ and $20 \dot{\wp} 41=63$;

(3) if $p=4 / 7$ and $q=9 / 14$, then additionally $\tilde{s}$ fulfils $P M$ on $10 \dot{\wp} 41=52,20 \dot{\wp} 30=52$ and $41 \dot{\wp} 41=65$;

(4) if $p=4 / 7$ and $q=19 / 28$, then $\tilde{s}$ also satisfies $P M$ on $10 \dot{\wp} 41=52,20 \dot{\wp} 41=63$ and $41 \dot{\wp} 41=65$; 
(5) if $p=4 / 7$ and $q=5 / 7$, then in addition $\tilde{s}$ fulfils $P M$ on $10 \dot{\wp} 41=52,10 \dot{\wp} 52=63$ and $41 \dot{\wp} 41=65$;

(6) if $p=7 / 12$ and $q=2 / 3$, then $\tilde{s}$ accomplishes $P M$ on $20 \dot{\wp} 30=52$ and $20 \dot{\wp} 41=63$.

Proof. We have that $W_{6}^{N_{1}}$ consists of 28 elements: $00<10<11,20<21<22,30<31<32<33$, $40<41<42<43<44,50<51<52<53<54<55,60<61<62<63<64<65<66$. An ordinary calculation shows that the set $E_{6}^{1}$ of all possible existing partial binary products is as follows:

$10 \dot{\wp} 10=21,10 \dot{\wp} 20=31,10 \dot{\wp} 21=32,10 \dot{\wp} 30=41,10 \dot{\wp} 31=42,10 \dot{\wp} 32=43,10 \dot{\wp} 40=51,10 \dot{\varphi} 41=52$, $10 \dot{\wp} 42=53,10 \dot{\wp} 43=54,10 \dot{\wp} 50=61,10 \dot{\wp} 51=62,10 \dot{\wp} 52=63,10 \dot{\wp} 53=64,10 \dot{\wp} 54=65,10 \dot{\wp} 65=66$, $11 \dot{\wp} 11=22,11 \dot{\wp} 22=33,11 \dot{\wp} 33=44,11 \dot{\wp} 44=55,11 \dot{\wp} 55=66,20 \dot{\wp} 20=42,20 \dot{\wp} 30=52,20 \dot{\wp} 31=53$, $20 \dot{\wp} 40=62,20 \dot{\wp} 41=63,20 \dot{\wp} 42=64,20 \dot{\wp} 53=65,20 \dot{\wp} 64=66,21 \dot{\wp} 21=43,21 \dot{\wp} 32=54,21 \dot{\wp} 43=65$, $21 \dot{\wp} 54=66,22 \dot{\wp} 22=44,22 \dot{\wp} 33=55,22 \dot{\wp} 44=66,30 \dot{\wp} 30=63,30 \dot{\wp} 41=64,30 \dot{\wp} 52=65,30 \dot{\wp} 63=66$, $31 \dot{\wp} 31=64,31 \dot{\wp} 42=65,31 \dot{\wp} 53=66,32 \dot{\wp} 32=65,32 \dot{\wp} 43=66,33 \dot{\wp} 33=66,40 \dot{\wp} 40=64,40 \dot{\wp} 51=65$, $40 \dot{\wp} 62=66,41 \dot{\wp} 41=65,41 \dot{\wp} 52=66,42 \dot{\wp} 42=66,50 \dot{\wp} 50=65,50 \dot{\wp} 61=66,51 \dot{\wp} 51=66,60 \dot{\wp} 60=66$ and $x \dot{\wp} 00=x$ for all $x \in \mathcal{W}_{6}^{N_{1}}$.

Now verifying each of the partial triple products in turn, we establish the following sixteen exclusive situations:

$$
\begin{aligned}
& \underbrace{(10 \dot{\wp} 20)}_{=31} \dot{\wp} 10=42 \text { is defined but } 20 \dot{\wp} \underbrace{(10 \dot{\wp} 10)}_{=21} \text { is not; } \\
& \underbrace{(10 \dot{\phi} 20)}_{=31} \dot{\wp} 42=20 \dot{\wp} \underbrace{(10 \dot{\phi} 42)}_{=53}=65 \text { are defined but } 10 \dot{\wp} \underbrace{(20 \dot{\varphi} 42)}_{=64} \text { is not; } \\
& \underbrace{(10 \dot{\beta} 30)}_{=41} \dot{\wp} 10=52 \text { is defined but } 30 \dot{\wp} \underbrace{(10 \dot{\wp} 10)}_{=21} \text { is not; } \\
& \underbrace{(10 \dot{\wp} 30)}_{=41} \dot{\wp} 20=10 \dot{\wp} \underbrace{(20 \dot{\wp} 30)}_{=52}=63 \text { are defined but } 30 \dot{\wp} \underbrace{(10 \dot{\beta} 20)}_{=31} \text { is not; } \\
& \underbrace{(10 \dot{\wp} 30)}_{=41} \dot{\wp} 30=64 \text { is defined but } 10 \dot{\wp} \underbrace{(30 \dot{\wp} 30)}_{=63} \text { is not; } \\
& \underbrace{(10 \dot{\wp} 30)}_{=41} \dot{\varphi} 41=30 \dot{\wp} \underbrace{(10 \dot{\varphi} 41)}_{=52}=65 \text { are defined but } 10 \dot{\wp} \underbrace{(30 \dot{\varphi} 41)}_{=64} \text { is not; } \\
& \underbrace{(10 \dot{\beta} 31)}_{=42} \dot{\wp} 10=53 \text { is defined but } 31 \dot{\wp} \underbrace{(10 \dot{\wp} 10)}_{=21} \text { is not; } \\
& \underbrace{(10 \dot{\wp} 31)}_{=42} \dot{\wp} 31=65 \text { is defined but } 10 \dot{\wp} \underbrace{(31 \dot{\wp} 31)}_{=64} \text { is not; } \\
& \underbrace{(10 \dot{\wp} 40)}_{=51} \dot{\wp} 10=62 \text { is defined but } 40 \dot{\wp} \underbrace{(10 \dot{\wp} 10)}_{=21} \text { is not; } \\
& \underbrace{(10 \dot{\varphi} 40)}_{=51} \dot{\varphi} 40=65 \text { is defined but } 10 \dot{\wp} \underbrace{(40 \dot{\varphi} 40)}_{64} \text { is not; } \\
& \underbrace{(10 \dot{\wp} 41)}_{=52} \dot{\wp} 10=63 \text { is defined but } 41 \dot{\wp} \underbrace{(10 \dot{\wp} 10)}_{=21} \text { is not; } \\
& \underbrace{(10 \dot{\wp} 41)}_{=52} \dot{\wp} 30=41 \dot{\wp} \underbrace{(10 \dot{\wp} 30)}_{=41}=65 \text { are defined but } 10 \dot{\wp} \underbrace{(30 \dot{\varphi} 41)}_{=64} \text { is not; } \\
& \underbrace{(10 \dot{\wp} 42)}_{=53} \dot{\wp} 10=64 \text { is defined but } 42 \dot{\wp} \underbrace{(10 \dot{\wp} 10)}_{=21} \text { is not; } \\
& \underbrace{(10 \dot{\wp} 42)}_{=53} \dot{\wp} 20=42 \dot{\varphi} \underbrace{(10 \dot{\wp} 20)}_{=31}=65 \text { are defined but } 10 \dot{\wp} \underbrace{(20 \dot{\varphi} 42)}_{=64} \text { is not; } \\
& \underbrace{(20 \dot{\wp} 30)}_{=52} \dot{\varphi} 10=20 \dot{\wp} \underbrace{(10 \dot{\wp} 30)}_{=41}=63 \text { are defined but } 30 \dot{\wp} \underbrace{(10 \dot{\varphi} 20)}_{=31} \text { is not; } \\
& \underbrace{(20 \dot{\beta} 30)}_{=52} \dot{\wp} 30=65 \text { is defined but } 20 \dot{\wp} \underbrace{(30 \dot{\wp} 30)}_{=63} \text { is not. }
\end{aligned}
$$


Further, gathering all binary products, we form the set $D_{6}^{1}$ of deflected partial binary products within $\mathcal{W}_{6}^{N_{1}}$; it consists of the following partial products as follows:

$10 \dot{\wp} 20=31,10 \dot{\wp} 30=41,10 \dot{\wp} 31=42,10 \dot{\wp} 40=51,10 \dot{\wp} 41=52,10 \dot{\wp} 42=53,10 \dot{\wp} 51=62,10 \dot{\wp} 52=63$, $10 \dot{\wp} 53=64,20 \dot{\wp} 30=52,20 \dot{\wp} 41=63,20 \dot{\wp} 53=65,30 \dot{\wp} 52=65,31 \dot{\wp} 42=65,40 \dot{\wp} 51=65$ and $41 \dot{\wp} 41=65$.

Now applying $\tilde{s}$ to all steady partial products within $\mathcal{W}_{6}^{N_{1}}$, we obtain the following system of linear equations:

$2 \tilde{s}_{10}=\tilde{s}_{21}, \tilde{s}_{10}+\tilde{s}_{21}=\tilde{s}_{32}, \tilde{s}_{10}+\tilde{s}_{32}=\tilde{s}_{43}, \tilde{s}_{10}+\tilde{s}_{43}=\tilde{s}_{54}, \tilde{s}_{10}+\tilde{s}_{50}=\tilde{s}_{61}, \tilde{s}_{10}+\tilde{s}_{54}=\tilde{s}_{65}, \tilde{s}_{10}+\tilde{s}_{65}=\tilde{s}_{66}, 2 \tilde{s}_{11}=\tilde{s}_{22}$, $\tilde{s}_{11}+\tilde{s}_{22}=\tilde{s}_{33}, \tilde{s}_{11}+\tilde{s}_{33}=\tilde{s}_{44}, \tilde{s}_{11}+\tilde{s}_{44}=\tilde{s}_{55}, \tilde{s}_{11}+\tilde{s}_{55}=\tilde{s}_{66}, 2 \tilde{s}_{20}=\tilde{s}_{42}, \tilde{s}_{20}+\tilde{s}_{31}=\tilde{s}_{53}, \tilde{s}_{20}+\tilde{s}_{40}=\tilde{s}_{62}$, $\tilde{s}_{20}+\tilde{s}_{42}=\tilde{s}_{64}, \tilde{s}_{20}+\tilde{s}_{64}=\tilde{s}_{66}, 2 \tilde{s}_{21}=\tilde{s}_{43}, \tilde{s}_{21}+\tilde{s}_{32}=\tilde{s}_{54}, \tilde{s}_{21}+\tilde{s}_{43}=\tilde{s}_{65}, \tilde{s}_{21}+\tilde{s}_{54}=\tilde{s}_{66}, 2 \tilde{s}_{22}=\tilde{s}_{44}, \tilde{s}_{22}+\tilde{s}_{33}=\tilde{s}_{55}$, $2 \tilde{s}_{30}=\tilde{s}_{63}, 2 \tilde{s}_{31}=\tilde{s}_{64}, \tilde{s}_{31}+\tilde{s}_{53}=\tilde{s}_{66}, 2 \tilde{s}_{32}=\tilde{s}_{65}, \tilde{s}_{32}+\tilde{s}_{43}=\tilde{s}_{66}, 2 \tilde{s}_{33}=\tilde{s}_{66}, 2 \tilde{s}_{40}=\tilde{s}_{64}, \tilde{s}_{40}+\tilde{s}_{62}=\tilde{s}_{66}$, $\tilde{s}_{41}+\tilde{s}_{52}=\tilde{s}_{66}, 2 \tilde{s}_{42}=\tilde{s}_{66}, 2 \tilde{s}_{50}=\tilde{s}_{65}, \tilde{s}_{50}+\tilde{s}_{61}=\tilde{s}_{66}, 2 \tilde{s}_{51}=\tilde{s}_{66}$ and $2 \tilde{s}_{60}=\tilde{s}_{66}$.

Note that $\tilde{s}_{00}=s_{0}=0, \tilde{s}_{11}=s_{1}=1 / 6, \tilde{s}_{22}=s_{2}=1 / 3, \tilde{s}_{33}=s_{3}=1 / 2, \tilde{s}_{44}=s_{4}=2 / 3, \tilde{s}_{55}=s_{5}=5 / 6$ and $\tilde{s}_{66}=s_{6}=1$. In view of this extension property, the analysis of the system of linear equations shows that it is not complete with $\tilde{s}_{52}=p$ and $\tilde{s}_{63}=q$ as parameters such that $1 / 2 \leq p \leq 5 / 8 \leq q \leq 3 / 4$ (by isotonicity of $\tilde{s}$ ). To supplement the system, we are looking for consistent equations by applying $\tilde{s}$ to suitable deflected partial products. We discover that

(1) the system of linear equations together with the equations $\tilde{s}_{10}+\tilde{s}_{30}=\tilde{s}_{41}$ and $\tilde{s}_{10}+\tilde{s}_{52}=\tilde{s}_{63}$;

(2) the system together with the equations $\tilde{s}_{10}+\tilde{s}_{52}=\tilde{s}_{63}$ and $\tilde{s}_{20}+\tilde{s}_{41}=\tilde{s}_{63}$;

(3) the system together with the equations $\tilde{s}_{20}+\tilde{s}_{30}=\tilde{s}_{52}$ and $2 \tilde{s}_{41}=\tilde{s}_{65}$;

(4) the system together with the equations $\tilde{s}_{20}+\tilde{s}_{41}=\tilde{s}_{63}$ and $2 \tilde{s}_{41}=\tilde{s}_{65}$;

(5) the system together with the equations $\tilde{s}_{10}+\tilde{s}_{52}=\tilde{s}_{63}$ and $2 \tilde{s}_{41}=\tilde{s}_{65}$;

(6) the system together with the equations $\tilde{s}_{20}+\tilde{s}_{30}=\tilde{s}_{52}$ and $\tilde{s}_{20}+\tilde{s}_{41}=\tilde{s}_{63}$

form six complete systems of linear equations yielding the required solutions. Finally, applying $\tilde{s}$ to all deflected partial binary products, we complete the proof.

Remark that the 1-extension $\mathcal{W}_{6}^{N_{1}}$ of the Weber $M V$-chain $\mathcal{W}_{6}$ contains the following seven Weber $M V$-chains:

$00<10<10^{2 \dot{\xi}}(=21)<10^{3 \dot{\phi}}(=32)<10^{4 \dot{\varphi}}(=43)<10^{5 \dot{\varphi}}(=54)<10^{6 \dot{\varphi}}(=65)<10^{7 \dot{\xi}}(=66), 00<11<11^{2 \dot{\varphi}}(=$ $22)<11^{3 \dot{\varphi}}(=33)<11^{4 \dot{\varphi}}(=44)<11^{5 \dot{\varphi}}(=55)<11^{6 \dot{\varphi}}(=66), 00<20<20^{2 \dot{\varphi}}(=42)<20^{3 \dot{\varphi}}(=64)<20^{4 \dot{\varphi}}(=66)$, $00<30<30^{2 \dot{\varphi}}(=63)<30^{3 \dot{\phi}}(=66), 00<42<42^{2 \dot{\varphi}}(=66), 00<51<51^{2 \dot{\xi}}(=66)$ and $00<60<60^{2 \dot{\xi}}(=66)$.

From Theorem 7 and the preceding proposition it follows that values of all fit states on $\mathcal{W}_{6}^{N_{1}}$ (except values $\tilde{s}_{40}$, $\tilde{s}_{41}, \tilde{s}_{50}, \tilde{s}_{52}, \tilde{s}_{61}, \tilde{s}_{62}$ and $\tilde{s}_{63}$ ) are in line with those of Weber state $W$. By definition of $W$, we have that it fulfils PM only on the following partial binary products:

$10 \dot{\wp} 10=21,10 \dot{\wp} 21=32,10 \dot{\wp} 32=43,10 \dot{\wp} 43=54,10 \dot{\wp} 54=65,10 \dot{\wp} 65=66,21 \dot{\wp} 21=43,21 \dot{\wp} 32=54$, $21 \dot{\wp} 43=65,21 \dot{\wp} 54=66,32 \dot{\wp} 32=65,32 \dot{\wp} 43=66,11 \dot{\wp} 11=22,11 \dot{\wp} 22=33,11 \dot{\wp} 33=44,11 \dot{9} 44=55$, $11 \dot{\wp} 55=66,22 \dot{\wp} 22=44,22 \dot{\wp} 33=55,22 \dot{\wp} 44=66,33 \dot{\wp} 33=66,20 \dot{\wp} 20=42,20 \dot{\wp} 42=64,20 \dot{\wp} 64=66$, $42 \dot{\wp} 42=66,30 \dot{\wp} 30=63,30 \dot{\wp} 63=66,42 \dot{\wp} 42=66,51 \dot{\wp} 51=66$ and 60ழ $60=66$;

by the preceding theorem everyone is steady product within $W_{6}^{\mathcal{N}_{1}}$. But this list of steady products is not full - the remainder is the following:

$10 \dot{\wp} 50=61,20 \dot{\wp} 20=42,20 \dot{\wp} 31=53,20 \dot{\wp} 40=62,20 \dot{\wp} 42=64,20 \dot{\wp} 64=66,31 \dot{\wp} 31=64,31 \dot{\wp} 53=66$, $40 \dot{\wp} 40=64,40 \dot{\wp} 62=66,41 \dot{\wp} 52=66,50 \dot{\wp} 50=65$ and $50 \dot{\wp} 61=66$.

Thus, all fit states on $\mathcal{W}_{6}^{N_{1}}$ fulfil PM on a much more large family of partial binary products than Weber state $W$ does.

Theorem 11 The unique state $s$ on $\mathcal{W}_{4}$, given by $s_{0}=0, s_{1}=1 / 4, s_{2}=1 / 2, s_{3}=3 / 4$ and $s_{4}=1$, has a unique fit state extension $\tilde{s}$ on $\mathcal{W}_{4}^{N_{2}}$ defined by

$\tilde{s}_{000}=0, \tilde{s}_{100}=1 / 6, \tilde{s}_{110}=5 / 24, \quad \tilde{s}_{111}=1 / 4, \quad \tilde{s}_{200}=1 / 4, \tilde{s}_{210}=1 / 3, \tilde{s}_{211}=3 / 8, \tilde{s}_{220}=3 / 8, \tilde{s}_{221}=5 / 12$, $\tilde{s}_{222}=1 / 2, \tilde{s}_{300}=5 / 18, \tilde{s}_{310}=5 / 12, \tilde{s}_{311}=5 / 12, \tilde{s}_{320}=5 / 12, \quad \tilde{s}_{321}=1 / 2, \tilde{s}_{322}=7 / 12, \tilde{s}_{330}=37 / 72$, $\tilde{s}_{331}=7 / 12, \tilde{s}_{332}=5 / 8, \tilde{s}_{333}=3 / 4, \tilde{s}_{400}=1 / 3, \tilde{s}_{410}=4 / 9, \tilde{s}_{411}=35 / 72, \tilde{s}_{420}=1 / 2, \tilde{s}_{421}=7 / 12, \tilde{s}_{422}=5 / 8$, $\tilde{s}_{430}=5 / 9, \tilde{s}_{431}=7 / 12, \tilde{s}_{432}=2 / 3, \tilde{s}_{433}=19 / 24, \tilde{s}_{440}=2 / 3, \tilde{s}_{441}=13 / 18, \tilde{s}_{442}=3 / 4, \tilde{s}_{443}=5 / 6$ and $\tilde{s}_{444}=1$. 
This unique fit state fulfils PM not only on all steady binary products but also on the following ten deflected binary products:

$100 \dot{\wp} 200=310,100 \dot{\wp} 221=322,100 \dot{\wp} 332=433,100 \dot{\wp} 431=442,110 \dot{\wp} 211=322,110 \dot{\wp} 220=331,110 \dot{\varnothing} 221=$ $332,110 \dot{\wp} 322=433,200 \dot{\wp} 431=443$ and $220 \dot{\wp} 311=433$,

while PM of $\tilde{s}$ cases to exist on the partial binary products:

$110 \dot{\varnothing} 200=311,110 \dot{\wp} 331=442,200 \dot{\wp} 310=431,200 \dot{\wp} 331=433$, and $310 \dot{\wp} 310=442$.

From now on we put all proofs in Appendix. As usual, the very details are tedious.

Theorem 12 The unique state $s$ on $\mathcal{W}_{5}$, given by $s_{0}=0, s_{1}=1 / 5, s_{2}=2 / 5, s_{3}=3 / 5, s_{4}=4 / 5$ and $s_{5}=1$, has an infinite family of fit state extensions $\tilde{s}$ on $W_{5}^{N_{2}}$ defined by

$\tilde{s}_{000}=0, \tilde{s}_{100}=1 / 7, \tilde{s}_{110}=6 / 35, \tilde{s}_{111}=1 / 5, \tilde{s}_{200}=3 / 14, \tilde{s}_{210}=2 / 7, \tilde{s}_{211}=11 / 35, \tilde{s}_{220}=(1-p) / 2, \tilde{s}_{221}=12 / 35$, $\tilde{s}_{222}=2 / 5, \tilde{s}_{300}=11 / 42, \tilde{s}_{310}=5 / 14, \tilde{s}_{311}=p, \tilde{s}_{320}=11 / 28, \tilde{s}_{321}=3 / 7, \tilde{s}_{322}=17 / 35, \tilde{s}_{330}=p / 2+5 / 21$, $\tilde{s}_{331}=p / 2+2 / 7, \tilde{s}_{332}=18 / 35, \tilde{s}_{333}=3 / 5, \tilde{s}_{400}=2 / 7, \tilde{s}_{410}=17 / 42, \quad \tilde{s}_{411}=q, \tilde{s}_{420}=3 / 7, \tilde{s}_{421}=1 / 2$, $\tilde{s}_{422}=5 / 7-p / 2, \tilde{s}_{430}=19 / 42, \tilde{s}_{431}=1 / 2, \tilde{s}_{432}=4 / 7, \tilde{s}_{433}=23 / 35, \tilde{s}_{440}=19 / 35, \tilde{s}_{441}=1-q, \tilde{s}_{442}=1-p$, $\tilde{s}_{443}=24 / 35, \tilde{s}_{444}=4 / 5, \tilde{s}_{500}=1 / 3, \tilde{s}_{510}=3 / 7, \tilde{s}_{511}=16 / 35, \tilde{s}_{520}=10 / 21, \tilde{s}_{521}=23 / 42, \tilde{s}_{522}=16 / 21-p / 2$, $\tilde{s}_{530}=11 / 21, \tilde{s}_{531}=4 / 7, \tilde{s}_{532}=17 / 28, \tilde{s}_{533}=(p+1) / 2, \tilde{s}_{540}=4 / 7, \tilde{s}_{541}=25 / 42, \tilde{s}_{542}=9 / 14, \tilde{s}_{543}=5 / 7$, $\tilde{s}_{544}=29 / 35, \tilde{s}_{550}=2 / 3, \tilde{s}_{551}=5 / 7, \tilde{s}_{552}=31 / 42, \tilde{s}_{553}=11 / 14, \tilde{s}_{554}=6 / 7, \tilde{s}_{555}=1$

and indexed by parameters $p$ and $q$ such that

$$
5 / 14 \leq p \leq q \leq 16 / 35, p \leq 3 / 7 \text { and } q \geq 17 / 42 .
$$

These fit states fulfil PM not only on all steady partial binary products but also on the following deflected partial

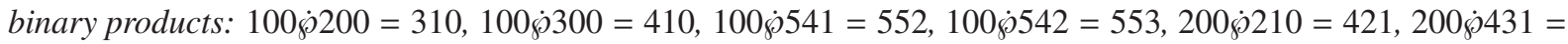
$543,200 \dot{\wp} 542=554,210 \dot{\wp} 431=553$ and $300 \dot{\wp} 541=554$. Moreover, among files of this family there are three fit states fulfilling PM further on the next deflected partial binary products:

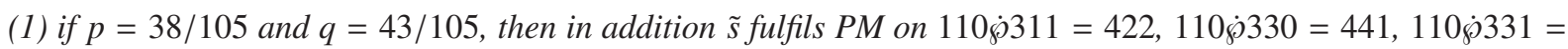
442, $110 \dot{\wp} 411=522,311 \dot{\wp} 331=544$ and $330 \dot{8} 411=544$;

(2) if $p=38 / 105$ and $q=13 / 30$, then $\tilde{s}$ fulfils $P M$ additionally on $110 \dot{\wp} 300=411,110 \dot{\wp} 311=422,110 \dot{\wp} 331=$ 442, $110 \dot{\wp} 441=552,300 \dot{\wp} 441=544$ and $311 \dot{\wp} 331=544$;

(3) if $p=27 / 70$ and $q=13 / 30$, then $\tilde{s}$ accomplishes PM further on $110 \dot{\wp} 200=311,110 \dot{\wp} 220=331,110 \dot{\wp} 300=$ 411, $110 \dot{\wp} 422=533,110 \dot{\wp} 441=552,110 \dot{\wp} 442=553,200 \dot{\wp} 442=544,220 \dot{\wp} 422=544$ and $300 \dot{\wp} 441=544$.

Theorem 13 The unique state $s$ on the Weber $M V$-chain $\mathcal{W}_{2}$ given by $s_{0}=0, s_{1}=1 / 2$ and $s_{2}=1$, has a unique fit state extension $\tilde{s}$ on 3-extension $\mathcal{W}_{2}^{\mathcal{N}_{3}}$ of $\mathcal{W}_{2}$ defined by

$\tilde{s}_{0000}=0, \tilde{s}_{1000}=1 / 5, \tilde{s}_{1100}=1 / 3, \tilde{s}_{1110}=2 / 5, \tilde{s}_{1111}=1 / 2, \tilde{s}_{2000}=1 / 4, \tilde{s}_{2100}=2 / 5, \tilde{s}_{2110}=1 / 2, \tilde{s}_{2111}=3 / 5$, $\tilde{s}_{2200}=1 / 2, \tilde{s}_{2210}=3 / 5, \tilde{s}_{2211}=2 / 3, \tilde{s}_{2220}=3 / 4, \tilde{s}_{2221}=4 / 5$ and $\tilde{s}_{2222}=1$.

Theorem 14 The unique state $s$ on the Weber $M V$-chain $\mathcal{W}_{3}$, given by $s_{0}=0, s_{1}=1 / 3, s_{2}=2 / 3$ and $s_{3}=1$, has an infinite family of fit state extensions $\tilde{s}$ on the 3-extension $\mathcal{W}_{3}^{\mathcal{N}_{3}}$ of $\mathcal{W}_{3}$ defined by

$\tilde{s}_{0000}=0, \tilde{s}_{1000}=1 / 6, \tilde{s}_{1100}=1 / 4, \tilde{s}_{1110}=5 / 18, \tilde{s}_{1111}=1 / 3, \tilde{s}_{2000}=5 / 24, \tilde{s}_{2100}=1 / 3, \tilde{s}_{2110}=5 / 12, \tilde{s}_{2111}=4 / 9$, $\tilde{s}_{2200}=3 / 8, \tilde{s}_{2210}=5 / 12, \tilde{s}_{2211}=1 / 2, \tilde{s}_{2220}=37 / 72, \tilde{s}_{2221}=5 / 9, \tilde{s}_{2222}=2 / 3, \tilde{s}_{3000}=1 / 4, \tilde{s}_{3100}=3 / 8, \tilde{s}_{3110}=p$, $\tilde{s}_{3111}=35 / 72, \tilde{s}_{3200}=5 / 12, \tilde{s}_{3210}=1 / 2, \tilde{s}_{3211}=7 / 12, \tilde{s}_{3220}=1-p, \tilde{s}_{3221}=7 / 12, \tilde{s}_{3222}=13 / 18, \tilde{s}_{3300}=1 / 2$, $\tilde{s}_{3310}=7 / 12, \tilde{s}_{3311}=5 / 8, \tilde{s}_{3320}=5 / 8, \tilde{s}_{3321}=2 / 3, \tilde{s}_{3322}=3 / 4, \tilde{s}_{3330}=3 / 4, \tilde{s}_{3331}=19 / 24, \tilde{s}_{3332}=5 / 6, \tilde{s}_{3333}=1$

and indexed by a parameter p located in the real interval [5/12,35/72]. These fit states fulfil PM also on several

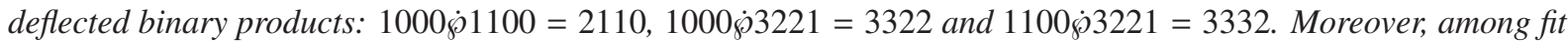
states of this family there is a state with $p=5 / 12$ fulfilling PM further on three deflected partial binary products:

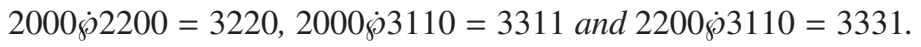

Theorem 15 The unique state $s$ on the Weber $M V$-chain $\mathcal{W}_{4}$, given by $s_{0}=0, s_{1}=1 / 4, s_{2}=1 / 2, s_{3}=3 / 4$ and $s_{4}=1$, has an infinite family of fit state extensions $\tilde{s}$ on the 3-extension $\mathcal{W}_{4}^{N_{3}}$ of $\mathcal{W}_{4}$ defined by

$\tilde{s}_{0000}=0, \tilde{s}_{1000}=1 / 7, \tilde{s}_{1100}=1 / 5, \tilde{s}_{1110}=3 / 14, \tilde{s}_{1111}=1 / 4, \tilde{s}_{2000}=1 / 5, \tilde{s}_{2100}=2 / 7, \tilde{s}_{2110}=9 / 28, \tilde{s}_{2111}=5 / 14$, $\tilde{s}_{2200}=1 / 3, \tilde{s}_{2210}=5 / 14, \tilde{s}_{2211}=2 / 5, \tilde{s}_{2220}=2 / 5, \tilde{s}_{2221}=3 / 7, \tilde{s}_{2222}=1 / 2, \tilde{s}_{3000}=3 / 14, \tilde{s}_{3100}=9 / 28$, $\tilde{s}_{3110}=53 / 140, \tilde{s}_{3111}=27 / 70, \tilde{s}_{3200}=5 / 14, \tilde{s}_{3210}=3 / 7, \tilde{s}_{3211}=13 / 28, \tilde{s}_{3220}=31 / 70, \tilde{s}_{3221}=1 / 2, \tilde{s}_{3222}=4 / 7$, 
$\tilde{s}_{3300}=2 / 5, \tilde{s}_{3310}=31 / 70, \tilde{s}_{3311}=1 / 2, \tilde{s}_{3320}=67 / 140, \tilde{s}_{3321}=15 / 28, \tilde{s}_{3322}=3 / 5, \tilde{s}_{3330}=4 / 7, \tilde{s}_{3331}=43 / 70$, $\tilde{s}_{3332}=9 / 14, \tilde{s}_{3333}=3 / 4, \tilde{s}_{4000}=1 / 4, \tilde{s}_{4100}=5 / 14, \tilde{s}_{4110}=1-p, \tilde{s}_{4111}=3 / 7, \tilde{s}_{4200}=2 / 5, \tilde{s}_{4210}=13 / 28$, $\tilde{s}_{4211}=73 / 140, \tilde{s}_{4220}=1 / 2, \tilde{s}_{4221}=39 / 70, \tilde{s}_{4222}=3 / 5, \tilde{s}_{4300}=3 / 7, \tilde{s}_{4310}=1 / 2, \tilde{s}_{4311}=39 / 70, \tilde{s}_{4320}=15 / 28$, $\tilde{s}_{4321}=4 / 7, \tilde{s}_{4322}=9 / 14, \tilde{s}_{4330}=p, \tilde{s}_{4331}=87 / 140, \tilde{s}_{4332}=19 / 28, \tilde{s}_{4333}=11 / 14, \tilde{s}_{4400}=1 / 2, \tilde{s}_{4410}=4 / 7$, $\tilde{s}_{4411}=3 / 5, \tilde{s}_{4420}=3 / 5, \tilde{s}_{4421}=9 / 14, \tilde{s}_{4422}=2 / 3, \tilde{s}_{4430}=9 / 14, \tilde{s}_{4431}=19 / 28, \tilde{s}_{4432}=5 / 7, \tilde{s}_{4433}=4 / 5$, $\tilde{s}_{4440}=3 / 4, \tilde{s}_{4441}=11 / 14, \tilde{s}_{4442}=4 / 5, \tilde{s}_{4443}=6 / 7$ and $\tilde{s}_{4444}=1$

indexed by a parameter $p$ located in the real interval [4/7,87/140]. These fit states fulfil PM in addition on the following deflected binary products: $1110 \wp 1110=2221,1110 \dot{\wp} 2111=3222,1110 \dot{\wp} 2220=3331,1110 \dot{\wp} 3111=$ 4222, $1110 \dot{\wp} 3222=4333,2111 \dot{\wp} 2221=4333$ and $2220 \dot{\wp} 3111=4333$. Moreover, if $p=41 / 70$, then $\tilde{s}$ satisfies PM additionally on $1100 \wp 3000=4110,1100 \wp 4330=4441$ and $3000 \wp 4330=4433$, while if $p=43 / 70$, then $\tilde{s}$ accomplishes PM further on $3000 \dot{\wp} 3300=4330,3000 \dot{8} 4110=4411$ and $3300 \dot{\wp} 4110=4441$.

Theorem 16 The unique state $s$ on the Weber $M V$-chain $\mathcal{W}_{2}$ given by $s_{0}=0, s_{1}=1 / 2$ and $s_{2}=1$, has an infinite family of fit state extensions $\tilde{s}$ on the 4-extension $\mathcal{W}_{2}^{\mathcal{N}_{4}}$ of $\mathcal{W}_{2}$ defined by

$\tilde{s}_{00000}=0, \tilde{s}_{10000}=1 / 6, \tilde{s}_{11000}=p, \tilde{s}_{11100}=(1-p) / 2, \tilde{s}_{11110}=5 / 12, \tilde{s}_{11111}=1 / 2, \tilde{s}_{20000}=1 / 5, \tilde{s}_{21000}=1 / 3$, $\tilde{s}_{21100}=1-2 p, \tilde{s}_{21110}=1 / 2, \tilde{s}_{2111}=7 / 12, \tilde{s}_{22000}=2 / 5, \tilde{s}_{22100}=1 / 2, \tilde{s}_{22110}=2 p, \tilde{s}_{22111}=(p+1) / 2, \tilde{s}_{22200}=3 / 5$, $\tilde{s}_{22210}=2 / 3, \tilde{s}_{22211}=1-p, \tilde{s}_{22220}=4 / 5, \tilde{s}_{22221}=5 / 6$ and $\tilde{s}_{22222}=1$,

where $p$ is a parameter located in the real interval $[1 / 4,1 / 3]$. Among these fit states there are three fit states:

(1) with $p=5 / 18$,

(2) with $p=7 / 24$, and

(3) with $p=1 / 3$,

fulfilling PM further on the next deflected partial binary products:

(1) if $p=5 / 18$, then PM occurs on products: $10000 \dot{\wp} 11000=21100,10000 \dot{\wp} 22110=22211$ and $11000 \dot{\wp} 22110=$ 22221;

(2) if $p=7 / 24$, then PM takes place on products: $10000 \dot{\wp} 21100=22110$ and $21100 \dot{\wp} 21100=22221$;

(3) if $p=1 / 3$, then PM holds on products: $10000 \dot{\wp} 11100=21110,10000 \dot{\wp} 21110=22111$ and $11100 \dot{\wp} 21110=$ 22221.

Theorem 17 The unique state $s$ on the Weber $M V$-chain $\mathcal{W}_{3}$, given by $s_{0}=0, s_{1}=1 / 3, s_{2}=2 / 3$ and $s_{3}=1$, has an infinite family of fit state extensions $\tilde{s}$ on 4-extension $\mathcal{W}_{3}^{N_{4}}$ of $\mathcal{W}_{3}$ defined by

$\tilde{s}_{00000}=0, \tilde{s}_{10000}=1 / 7, \tilde{s}_{11000}=3 / 14, \tilde{s}_{11100}=11 / 42, \tilde{s}_{11110}=2 / 7, \tilde{s}_{11111}=1 / 3, \tilde{s}_{20000}=6 / 35, \tilde{s}_{21000}=2 / 7$, $\tilde{s}_{21100}=5 / 14, \tilde{s}_{21110}=17 / 42, \tilde{s}_{21111}=3 / 7, \tilde{s}_{22000}=43 / 140, \tilde{s}_{22100}=11 / 28, \tilde{s}_{22110}=3 / 7, \tilde{s}_{22111}=10 / 21$, $\tilde{s}_{22200}=181 / 420, \tilde{s}_{22210}=19 / 42, \tilde{s}_{22211}=11 / 21, \tilde{s}_{22220}=19 / 35, \tilde{s}_{22221}=4 / 7, \tilde{s}_{22222}=2 / 3, \tilde{s}_{30000}=1 / 5$, $\tilde{s}_{31000}=11 / 35, \tilde{s}_{31100}=27 / 70, \tilde{s}_{31110}=p, \tilde{s}_{31111}=16 / 35, \tilde{s}_{32000}=12 / 35, \tilde{s}_{32100}=3 / 7, \tilde{s}_{32110}=1 / 2, \tilde{s}_{32111}=$ $23 / 42, \tilde{s}_{32200}=67 / 140, \tilde{s}_{32210}=1 / 2, \tilde{s}_{32211}=4 / 7, \tilde{s}_{32220}=1-p, \tilde{s}_{32221}=25 / 42, \tilde{s}_{32222}=5 / 7, \tilde{s}_{33000}=2 / 5$, $\tilde{s}_{33100}=17 / 35, \tilde{s}_{33110}=73 / 140, \tilde{s}_{33111}=239 / 420, \tilde{s}_{33200}=18 / 35, \tilde{s}_{33210}=4 / 7, \tilde{s}_{33211}=17 / 28, \tilde{s}_{33220}=43 / 70$, $\tilde{s}_{33221}=9 / 14, \tilde{s}_{33222}=31 / 42, \tilde{s}_{33300}=3 / 5, \tilde{s}_{33310}=23 / 35, \tilde{s}_{33311}=97 / 140, \tilde{s}_{33320}=24 / 35, \tilde{s}_{33321}=5 / 7$, $\tilde{s}_{33322}=11 / 14, \tilde{s}_{33330}=4 / 5, \tilde{s}_{33331}=29 / 35, \tilde{s}_{33332}=6 / 7$ and $\tilde{s}_{33333}=1$,

where $p$ is a parameter located in the real interval [17/42,16/35]. These fit states fulfil PM not only on all steady partial binary products but also on the following deflected partial binary products:

$10000 \dot{\wp} 11000=21100,10000 \dot{\wp} 11100=21110,10000 \dot{\wp} 32221=33222,10000 \dot{\wp} 33221=33322,11000 \dot{\varnothing} 20000=$ $31100,11000 \dot{\wp} 21000=32110,11000 \dot{\wp} 32210=33321,11000 \dot{8} 32211=33322,11000 \dot{\wp} 33220=33331$, $11000 \dot{8} 33221=33332,11100 \dot{\wp} 32221=33332,20000 \dot{\wp} 22000=32200,20000 \dot{\wp} 33110=33311,20000 \dot{\wp} 33220=$ $33322,21000 \dot{\wp} 32210=33322$ and $22000 \dot{\wp} 33110=33331$.

Moreover, if $p=13 / 30$, then $\tilde{s}$ accomplishes $P M$ further on $11100 \dot{\wp} 20000=31110,11100 \dot{\wp} 32220=33331$ and $20000 \dot{0} 32220=33222$.

\section{Discussion}

In this paper we have proposed a new notion of fit states on a Girard algebra opposing to Weber's concept of weakly additive states which is based on additivity only for all sub- $M V$-algebras. That artificial axiom permits to Weber to obtain a complete characterization of all weakly additive states on the canonical extension $\mathcal{W}_{m}^{\mathcal{N}_{1}}$ of any finite 
$M V$-chain $\mathcal{W}_{m}$ and to express values of a state by an imposing formula (see Theorem 7).

In our paper there is almost nothing done like Weber did because we have needed to go very far in order a posteriori to understand the nature of a state on each $n$-extension $\mathcal{W}_{m}^{\mathcal{N}_{n}}$ in a separate way. Facts presented in the ends of Proof of Theorem 12 and of Proof of Theorem 17 reinforce our doubts about Weber's original choice.

It is natural to examine fit states on products of $n$-extensions $\mathcal{W}_{m}^{\mathcal{N}_{n}}$ and to calculate these entities. Since this theme is beyond the scope of the present paper, we only quote second research by Weber (2010) and paper by Gylys (2012). But the main challenge is a "move" towards non-commutating generalizations of Girard algebras developed especially in Slovak and Czech Schools. However, this stumbles on the lack of non-trivial principal examples and models of such structures.

\section{References}

Dvurečenskij, A., \& Pulmanova, S. (2000). New Trends in Quantum Structures. Kluwer Academic Publishers, Ister Science, Dordrecht, Bratislava.

Girard, J.-Y. (2004). Between logic and quantic: A tract. In Ruet, Ehrhard, Girard, (\&) Scott (Ed.), Linear logic in Computer Science, CUP, pp. 346-381.

Goodman, I. R., Nguyen, H. T., \& Walker, E. A. (1991). Conditional Inference and Logic for Intelligent Systems: A Theory of Measure-Free Conditioning. North-Holland,Amsterdam.

Gylys, R. P. (2010). Effectible residuated lattices and n-th roots. Fuzzy Sets Syst., 161(12), 1676-1698. http://dx.doi.org/10.1016/j.fss.2009.12.009

Gylys, R. P. (2012). Extensions of states on MV-quantales. Fuzzy Sets Syst., 194, 31-51. http://dx.doi.org/10.1016/j.fss.2011.11.007

Höhle, U., \& Weber, S. (1997). Uncertainty measures, realizations and entropies. In J. Gontsias, R. P. S. Mahler, \& H. T. Nguyen (Eds.), Random Sets: Theory and Applications (pp. 259-295). Heidelberg/Berlin/New York: Springer-Verlag.

Höhle, U., (\&) Weber, S. (1999). On conditioning operators. In U. Höhle, \& S. E. Rodabaugh (Eds.), Mathematics of Fuzzy Sets (pp. 653-673). Boston/Dordrecht/London: Kluwer Academic Publishers.

Weber, S. (1997). Conditioning on $M V$-algebras and additive measures, Part I. Fuzzy Sets Syst., 92, 241-250.

Weber, S. (1999). Conditioning on $M V$-algebras and additive measures - further results. In D. Dubois, H. Prade, \& E. P. Klement (Eds.), Fuzzy Sets, Logics and Reasoning about Knowledge (pp. 175-199). Kluwer Academic Publishers, Boston/Dordrecht.

Weber, S. (2009). Uncertainty measures-problems concerning additivity. Fuzzy Sets Syst. 160(3), $371-383$. http://dx.doi.org/10.1016/j.fss.2008.09.019

Weber, S. (2010a). A complete characterization of all weakly additive measures and of all valuations on the canonical extension of any finite MV-chain. Fuzzy Sets Syst. 161(9), 1350-1367. http://dx.doi.org/10.1016/j.fss.2009.06.004

Weber, S. (2010b). Measure-free conditioning and extensions of additive measures on finite $M V$-algebras. Fuzzy Sets Syst. 161(18), 2479-2504. http://dx.doi.org/10.1016/j.fss.2010.04.011 


\section{Appendix}

In this appendix we present proofs of Theorem 11, Theorem 12, Theorem 13, Theorem 14, Theorem 15, Theorem 16, and also of Theorem 17.

\section{Proof of Theorem 11}

We have that $W_{4}^{N_{2}}$ consists of 35 elements: $000<100<110<111,200<210<211,220<221<222$, $300<310<311,320<321<322,330<331<332<333,400<410<411,420<421<422$, $430<431<432<433,440<441<442<443<444$. A routine calculation shows that the set $E_{4}^{2}$ of all existing partial binary products within $\mathcal{W}_{4}^{N_{2}}$ is as follows:

$100 \dot{\wp} 100=210,100 \dot{\wp} 110=211,100 \dot{\wp} 200=310,100 \dot{\wp} 210=321,100 \dot{\wp} 221=322,100 \dot{\wp} 300=410,100 \dot{\wp} 310=$ $421,100 \dot{\wp} 320=431,100 \wp 321=432,100 \dot{\wp} 332=433,100 \wp 430=441,100 \dot{\wp} 431=442,100 \wp 432=443$, $100 \dot{\varnothing} 443=444,110 \dot{\wp} 110=221,110 \dot{\wp} 200=311,110 \dot{\wp} 211=322,110 \dot{\wp} 220=331,110 \dot{\wp} 221=332,110 \dot{\wp} 300=$

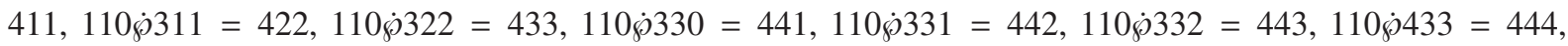
$111 \dot{\wp} 111=222,111 \dot{\wp} 222=333,111 \dot{\wp} 333=444,200 \dot{\wp} 200=420,200 \dot{\wp} 210=421,200 \dot{\wp} 220=422,200 \dot{\wp} 310=$ $431,200 \dot{\wp} 320=432,200 \dot{\wp} 331=433,200 \dot{\wp} 420=442,200 \dot{\wp} 431=443,200 \dot{\varphi} 442=444,210 \dot{\wp} 210=432$, $210 \dot{\wp} 320=442,210 \dot{\wp} 321=443,210 \dot{\wp} 432=444,211 \dot{\wp} 332=444,220 \dot{\wp} 220=442,220 \dot{\wp} 311=433,220 \dot{\wp} 422=$ $444,221 \dot{\wp} 221=443,221 \dot{\wp} 322=444,222 \dot{\wp} 222=444,300 \wp 300=430,300 \dot{\wp} 330=433,300 \wp 410=441$,

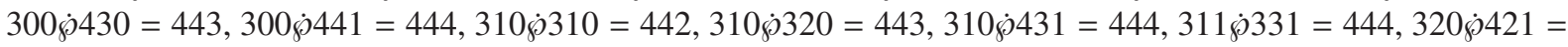
$444,321 \dot{\wp} 321=444,330 \dot{\wp} 411=444,400 \dot{\wp} 400=440,400 \dot{\wp} 440=444,410 \dot{\wp} 430=444,420 \dot{\wp} 420=444$ and $x \dot{\wp} 000=x$ for all $x \in \mathcal{W}_{4}^{N_{2}}$.

Now verifying each of the partial triple products in turn, we establish the following seven exclusive situations:

$$
\begin{aligned}
& \underbrace{(100 \dot{8} 200)}_{=310} \dot{\wp} 310=100 \dot{\wp} \underbrace{(200 \dot{\beta} 310)}_{=431}=442 \text { are defined but } 200 \dot{\wp} \underbrace{(100 \dot{\wp} 310)}_{=421} \text { is not; } \\
& \underbrace{(110 \dot{\wp} 211)}_{=322} \dot{\wp} 110=433 \text { is defined but } 211 \dot{\wp} \underbrace{(110 \dot{\wp} 110)}_{=221} \text { is not; } \\
& \underbrace{(110 \dot{\wp} 220)}_{=331} \dot{\wp} 110=442 \text { is defined but } 220 \dot{\wp} \underbrace{(110 \dot{\wp} 110)}_{=221} \text { is not; } \\
& \underbrace{(110 \dot{\wp} 221)}_{=332} \dot{\wp} 100=110 \dot{\wp} \underbrace{(100 \dot{\wp} 221)}_{=322}=433 \text { are defined but } 221 \dot{\wp} \underbrace{(100 \dot{\wp} 110)}_{=211} \text { is not; } \\
& \underbrace{(200 \dot{\wp} 310)}_{=431} \dot{\wp} 200=443 \text { is defined but } 310 \dot{\wp} \underbrace{(200 \dot{\varphi} 200)}_{=420} \text { is not; } \\
& \underbrace{(100 \dot{\wp} 200)}_{=310} \dot{\wp} 200=431 \text { is defined but } 100 \dot{\wp} \underbrace{(200 \dot{8} 200)}_{=420} \text { is not; } \\
& \underbrace{(110 \dot{\wp} 200)}_{=311} \dot{\varnothing} 220=200 \dot{\wp} \underbrace{(110 \dot{\varnothing} 220)}_{=331}=433 \text { are defined but } 110 \dot{\wp} \underbrace{(200 \dot{\wp} 220)}_{=422} \text { is not. }
\end{aligned}
$$

From this it follows that the set $D_{4}^{2}$ of all deflected partial binary products within $W_{4}^{\mathcal{N}_{2}}$ is as follows:

$100 \dot{\wp} 200=310,100 \dot{\wp} 221=322,100 \dot{\wp} 332=433,100 \dot{\varphi} 431=442,110 \dot{\wp} 200=311,110 \dot{\wp} 211=322,110 \dot{\wp} 220=$ $331,110 \dot{\wp} 221=332,110 \dot{\wp} 322=433,110 \dot{\wp} 331=442,200 \wp 310=431,200 \dot{\wp} 331=433,200 \dot{\wp} 431=443$, $220 \dot{\wp} 311=433$ and $310 \dot{\wp} 310=442$.

Now applying $\tilde{s}$ to all steady products within $W_{4}^{\mathcal{N}_{2}}$, we obtain the following system of linear equations:

$2 \tilde{s}_{100}=\tilde{s}_{210}, \tilde{s}_{100}+\tilde{s}_{110}=\tilde{s}_{211}, \tilde{s}_{100}+\tilde{s}_{210}=\tilde{s}_{321}, \tilde{s}_{100}+\tilde{s}_{300}=\tilde{s}_{410}, \tilde{s}_{100}+\tilde{s}_{310}=\tilde{s}_{421}, \tilde{s}_{100}+\tilde{s}_{320}=\tilde{s}_{431}$, $\tilde{s}_{100}+\tilde{s}_{321}=\tilde{s}_{432}, \tilde{s}_{100}+\tilde{s}_{430}=\tilde{s}_{441}, \tilde{s}_{100}+\tilde{s}_{432}=\tilde{s}_{443}, \tilde{s}_{100}+\tilde{s}_{443}=\tilde{s}_{444}, 2 \tilde{s}_{110}=\tilde{s}_{221}, \tilde{s}_{110}+\tilde{s}_{300}=\tilde{s}_{411}$, $\tilde{s}_{110}+\tilde{s}_{311}=\tilde{s}_{422}, \tilde{s}_{110}+\tilde{s}_{330}=\tilde{s}_{441}, \tilde{s}_{110}+\tilde{s}_{332}=\tilde{s}_{443}, \tilde{s}_{110}+\tilde{s}_{433}=\tilde{s}_{444}, 2 \tilde{s}_{111}=\tilde{s}_{222}, \tilde{s}_{111}+\tilde{s}_{222}=\tilde{s}_{333}$, $\tilde{s}_{111}+\tilde{s}_{333}=\tilde{s}_{444}, 2 \tilde{s}_{200}=\tilde{s}_{420}, \tilde{s}_{200}+\tilde{s}_{210}=\tilde{s}_{421}, \tilde{s}_{200}+\tilde{s}_{220}=\tilde{s}_{422}, \tilde{s}_{200}+\tilde{s}_{320}=\tilde{s}_{432}, \tilde{s}_{200}+\tilde{s}_{420}=\tilde{s}_{442}$, $\tilde{s}_{200}+\tilde{s}_{442}=\tilde{s}_{444}, 2 \tilde{s}_{210}=\tilde{s}_{432}, \tilde{s}_{210}+\tilde{s}_{320}=\tilde{s}_{442}, \tilde{s}_{210}+\tilde{s}_{321}=\tilde{s}_{443}, \tilde{s}_{210}+\tilde{s}_{432}=\tilde{s}_{444}, \tilde{s}_{211}+\tilde{s}_{332}=\tilde{s}_{444}$, $2 \tilde{s}_{220}=\tilde{s}_{442}, \tilde{s}_{220}+\tilde{s}_{422}=\tilde{s}_{444}, 2 \tilde{s}_{221}=\tilde{s}_{443}, \tilde{s}_{221}+\tilde{s}_{322}=\tilde{s}_{444}, 2 \tilde{s}_{222}=\tilde{s}_{444}, 2 \tilde{s}_{300}=\tilde{s}_{430}, \tilde{s}_{300}+\tilde{s}_{330}=\tilde{s}_{433}$, $\tilde{s}_{300}+\tilde{s}_{410}=\tilde{s}_{441}, \tilde{s}_{300}+\tilde{s}_{430}=\tilde{s}_{443}, \tilde{s}_{300}+\tilde{s}_{441}=\tilde{s}_{444}, \tilde{s}_{310}+\tilde{s}_{320}=\tilde{s}_{443}, \tilde{s}_{310}+\tilde{s}_{431}=\tilde{s}_{444}, \tilde{s}_{311}+\tilde{s}_{331}=\tilde{s}_{444}$, $\tilde{s}_{320}+\tilde{s}_{421}=\tilde{s}_{444}, 2 \tilde{s}_{321}=\tilde{s}_{444}, \tilde{s}_{330}+\tilde{s}_{411}=\tilde{s}_{444}, 2 \tilde{s}_{400}=\tilde{s}_{440}, \tilde{s}_{400}+\tilde{s}_{440}=\tilde{s}_{444}, \tilde{s}_{410}+\tilde{s}_{430}=\tilde{s}_{444}$ and $2 \tilde{s}_{420}=\tilde{s}_{444}$. 
Next, in view of the extension property: $\tilde{s}_{000}=s_{0}=0, \tilde{s}_{111}=s_{1}=1 / 4, \tilde{s}_{222}=s_{2}=1 / 2, \tilde{s}_{333}=s_{3}=3 / 4$ and $\tilde{s}_{444}=s_{4}=1$, we obtain the required solutions. Finally, applying $\tilde{s}$ to all deflected partial binary products, we obtain that

$\tilde{s}_{100}+\tilde{s}_{200}=\tilde{s}_{310}, \tilde{s}_{100}+\tilde{s}_{221}=\tilde{s}_{322}, \tilde{s}_{100}+\tilde{s}_{332}=\tilde{s}_{433}, \tilde{s}_{100}+\tilde{s}_{431}=\tilde{s}_{442}, \tilde{s}_{110}+\tilde{s}_{200} \neq \tilde{s}_{311}, \tilde{s}_{110}+\tilde{s}_{211}=\tilde{s}_{322}$, $\tilde{s}_{110}+\tilde{s}_{220}=\tilde{s}_{331}, \tilde{s}_{110}+\tilde{s}_{221}=\tilde{s}_{332}, \tilde{s}_{110}+\tilde{s}_{322}=\tilde{s}_{433}, \tilde{s}_{110}+\tilde{s}_{331} \neq \tilde{s}_{442}, \tilde{s}_{200}+\tilde{s}_{310} \neq \tilde{s}_{431}, \tilde{s}_{200}+\tilde{s}_{331} \neq \tilde{s}_{433}$, $\tilde{s}_{200}+\tilde{s}_{431}=\tilde{s}_{443}, \tilde{s}_{220}+\tilde{s}_{311}=\tilde{s}_{433}$ and $2 \tilde{s}_{310} \neq \tilde{s}_{442}$.

From this, it follows the assertion.

Note that the 2-extension $\mathcal{W}_{4}^{\mathcal{N}_{2}}$ of the Weber $M V$-chain $\mathcal{W}_{4}$ contains the following seven Weber $M V$-chains:

$000<100<100^{2 \dot{\beta}}(=210)<100^{3 \dot{\varphi}}(=321)<100^{4 \dot{\xi}}(=432)<100^{5 \dot{\beta}}(=443)<100^{6 \dot{\varphi}}(=444), 000<111<$ $111^{2 \dot{\varphi}}(=222)<111^{3 \dot{\varphi}}(=333)<111^{4 \dot{\varphi}}(=444), 000<200<200^{2 \dot{\dot{\varphi}}}(=420)<200^{3 \dot{\dot{\varphi}}}(=442)<200^{4 \dot{\varphi}}(=444)$, $000<210<210^{2 \dot{\phi}}(=432)<210^{3 \dot{\phi}}(=444), 000<321<321^{2 \dot{\phi}}(=444), 000<400<400^{2 \dot{\phi}}(=440)<400^{3 \dot{\phi}}(=$ 444) and $000<420 \leq 420^{2 \dot{\varphi}}(=444)$.

\section{Proof of Theorem 12}

We have that $W_{5}^{N_{2}}$ consists of 56 elements: $000<100<110<111,200<210<211,220<221<222$, $300<310<311,320<321<322,330<331<332<333,400<410<411,420<421<422,430<431<$ $432<433,440<441<442<443<444,500<510<511,520<521<522,530<531<532<533$, $540<541<542<543<544,550<551<552<553<554<555$. An ordinary calculation shows that the set $E_{5}^{2}$ of all possible existing partial binary products within $\mathcal{W}_{5}^{\mathcal{N}_{2}}$ is as follows:

$100 \dot{1} 100=210,100 \wp 110=211,100 \dot{2} 200=310,100 \dot{8} 210=321,100 \dot{2} 221=322,100 \wp 300=410,100 \dot{\wp} 310=$ $421,100 \dot{\wp} 320=431,100 \dot{\wp} 321=432,100 \dot{\wp} 332=433,100 \wp 400=510,100 \dot{\wp} 410=521,100 \wp 420=531$, $100 \dot{\wp} 421=532,100 \wp 430=541,100 \dot{\wp} 431=542,100 \wp 432=543,100 \dot{\wp} 443=544,100 \dot{\wp} 540=551,100 \dot{\wp} 541=$

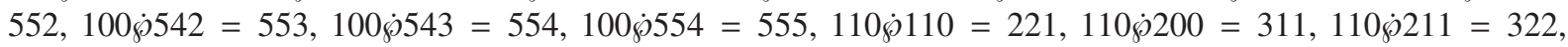

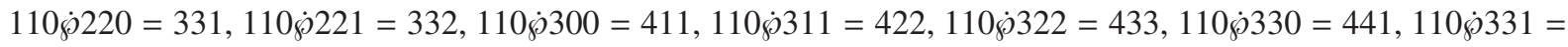
$442,110 \dot{\wp} 332=443,110 \dot{\wp} 400=511,110 \dot{\wp} 411=522,110 \dot{\wp} 422=533,110 \dot{\wp} 433=544,110 \dot{\wp} 440=551$, $110 \dot{\wp} 441=552,110 \dot{\wp} 442=553,110 \dot{\wp} 443=554,110 \dot{\wp} 544=555,111 \dot{\wp} 111=222,111 \dot{\wp} 222=333,111 \dot{\wp} 333=$ $444,111 \dot{\wp} 444=555,200 \dot{\wp} 200=420,200 \dot{2} 210=421,200 \dot{8} 220=422,200 \dot{\beta} 300=520,200 \dot{\wp} 310=531$, $200 \dot{\wp} 320=532,200 \wp 331=533,200 \dot{\wp} 420=542,200 \wp 431=543,200 \dot{\wp} 442=544,200 \wp 530=552,200 \dot{\wp} 531=$

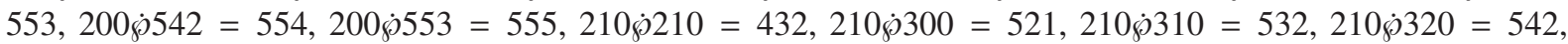
$210 \dot{\wp} 321=543,210 \dot{\wp} 430=552,210 \dot{\wp} 431=553,210 \dot{\wp} 432=554,210 \dot{\wp} 543=555,211 \dot{\wp} 221=433,211 \dot{\wp} 332=$ $544,211 \dot{\wp} 443=555,220 \dot{\wp} 220=442,220 \dot{\wp} 300=522,220 \wp 311=533,220 \dot{\wp} 330=552,220 \wp 331=553$, $220 \dot{\wp} 422=544,220 \dot{\wp} 533=555,221 \dot{\wp} 221=443,221 \dot{3} 322=544,221 \dot{\wp} 332=554,221 \dot{\varphi} 433=555,222 \dot{\wp} 222=$ $444,222 \dot{\wp} 333=555,300 \dot{\wp} 300=530,300 \dot{\wp} 330=533,300 \dot{\wp} 410=541,300 \dot{\wp} 430=543,300 \dot{\wp} 441=544$,

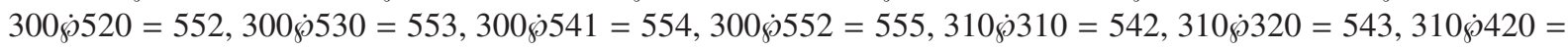
$553,310 \dot{\varnothing} 431=554,310 \dot{\wp} 542=555,311 \dot{\wp} 331=544,311 \dot{\wp} 442=555,320 \dot{\wp} 320=553,320 \dot{\varnothing} 421=554$,

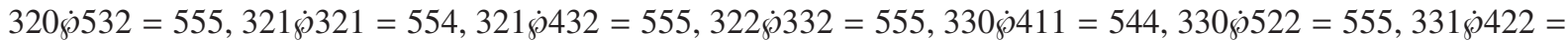
$555,400 \dot{\wp} 400=540,400 \dot{\wp} 440=544,400 \dot{\wp} 510=551,400 \dot{\wp} 540=554,400 \dot{\wp} 551=555,410 \dot{\wp} 410=552$,

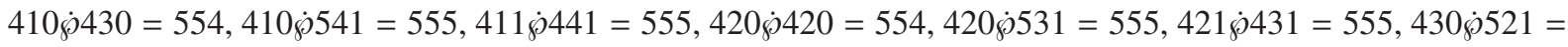
$555,440 \dot{\wp} 511=555,500 \dot{\wp} 500=550,500 \dot{\wp} 550=555,510 \dot{\wp} 540=555,520 \dot{\wp} 530=555$ and $x \dot{\wp} 000=x$ for all $x \in \mathcal{W}_{5}^{\mathrm{N}_{2}}$.

Now verifying each of the partial triple products in turn, we find the following twenty seven "non-associative" situations:

$$
\underbrace{(100 \dot{\wp} 200)}_{=310} \dot{\wp} 210=100 \dot{\wp} \underbrace{(200 \dot{\wp} 210)}_{=421}=532 \text { are defined but } 200 \dot{\wp} \underbrace{(100 \dot{\wp} 210)}_{=321} \text { is not; }
$$

$\underbrace{(100 \dot{\wp} 200)}_{=310} \dot{\wp} 310=542$ is defined but $100 \dot{\wp} \underbrace{(200 \dot{\wp} 310)}_{=531}$ and $200 \dot{\wp} \underbrace{(100 \dot{\wp} 310)}_{=421}$ are not;

$\underbrace{(100 \dot{\phi} 200)}_{=310} \dot{\wp} 320=200 \dot{\wp} \underbrace{(100 \dot{\wp} 320)}_{=431}=543$ are defined but $100 \dot{\wp} \underbrace{(200 \dot{\wp} 320)}_{=532}$ is not;

$\underbrace{(100 \dot{\wp} 300)}_{=410} \dot{\wp} 300=541$ is defined but $100 \dot{\wp} \underbrace{(300 \dot{\wp} 300)}_{=530}$ is not;

$\underbrace{(100 \dot{\wp} 300)}_{=410} \dot{\wp} 410=100 \dot{\wp} \underbrace{(300 \dot{\wp} 410)}_{=541}=552$ are defined but $300 \dot{\wp} \underbrace{(100 \dot{\wp} 410)}_{=521}$ is not; 
$\underbrace{(100 \dot{\wp} 320)}_{=431} \dot{\wp} 200=320 \dot{\wp} \underbrace{(100 \dot{\wp} 200)}_{=310}=543$ are defined but $100 \dot{\wp} \underbrace{(200 \dot{\wp} 320)}_{=532}$ is not;
$\underbrace{(100 \dot{\wp} 320)}_{=431} \dot{\wp} 210=100 \dot{\dot{\theta}} \underbrace{(210 \dot{\phi} 320)}_{=542}=553$ are defined but $320 \dot{\wp} \underbrace{(100 \dot{\wp} 210)}_{=321}$ is not; $\underbrace{(110 \dot{\wp} 200)}_{=311} \dot{\wp} 110=422$ is defined but $200 \dot{\wp} \underbrace{(110 \dot{\wp} 110)}_{=221}$ is not;

$\underbrace{(110 \dot{\phi} 200)}_{=311} \dot{\wp} 331=200 \dot{\phi} \underbrace{(110 \dot{\phi} 331)}_{=442}=544$ are defined but $110 \dot{\wp} \underbrace{(200 \dot{\phi} 331)}_{=533}$ is not; $\underbrace{(110 \dot{\wp} 220)}_{=331} \dot{\wp} 110=442$ is defined but $220 \dot{\wp} \underbrace{(110 \dot{\wp} 110)}_{=221}$ is not;

$\underbrace{(110 \dot{\wp} 220)}_{=331} \dot{\wp} 311=220 \dot{\wp} \underbrace{(110 \dot{\wp} 311)}_{=422}=544$ are defined but $110 \dot{\wp} \underbrace{(220 \dot{\wp} 311)}_{=533}$ is not; $\underbrace{(110 \dot{\wp} 300)}_{=411} \dot{\wp} 110=522$ is defined but $300 \dot{\wp} \underbrace{(110 \dot{\wp} 110)}_{=221}$ is not;

$\underbrace{(110 \dot{\wp} 300)}_{=411} \dot{\wp} 330=300 \dot{\wp} \underbrace{(110 \dot{8} 330)}_{=441}=544$ are defined but $110 \dot{\phi} \underbrace{(300 \dot{\varnothing} 330)}_{=533}$ is not; $\underbrace{(110 \dot{\wp} 311)}_{=422} \dot{\wp} 110=533$ is defined but $311 \dot{\wp} \underbrace{(110 \dot{\wp} 110)}_{=221}$ is not;

$\underbrace{(110 \dot{\phi} 311)}_{=422} \dot{\wp} 220=311 \dot{\phi} \underbrace{(110 \dot{\phi} 220)}_{=331}=544$ are defined but $110 \dot{\wp} \underbrace{(220 \dot{\phi} 311)}_{=533}$ is not; $\underbrace{(110 \dot{\wp} 330)}_{=441} \dot{\wp} 110=552$ is defined but $330 \dot{\wp} \underbrace{(110 \dot{\wp} 110)}_{=221}$ is not;

$\underbrace{(110 \dot{\ominus} 330)}_{=441} \dot{\varphi} 300=330 \dot{\wp} \underbrace{(110 \dot{\varnothing} 300)}_{=411}=544$ are defined but $110 \dot{\oint} \underbrace{(300 \dot{\ominus} 330)}_{=533}$ is not; $\underbrace{(110 \dot{\wp} 331)}_{=442} \dot{\wp} 110=553$ is defined but $331 \dot{\wp}(\underbrace{(110 \dot{\wp} 110)}_{=221}$ is not;

$\underbrace{(110 \dot{\wp} 331)}_{=442} \dot{\wp} 200=331 \dot{\wp} \underbrace{(110 \dot{\varnothing} 200)}_{=311}=544$ are defined but $110 \dot{\wp} \underbrace{(200 \dot{\varnothing} 331)}_{=533}$ is not; $\underbrace{(200 \dot{\wp} 210)}_{=421} \dot{\wp} 100=210 \dot{\wp} \underbrace{(100 \dot{\wp} 200)}_{=310}=532$ are defined but $200 \dot{\wp} \underbrace{(100 \dot{\wp} 210)}_{=321}$ is not; $\underbrace{(200 \dot{\wp} 210)}_{=421} \dot{\wp} 320=200 \dot{\wp} \underbrace{(210 \dot{\wp} 320)}_{=542}=554$ are defined but $210 \dot{\wp} \underbrace{(200 \dot{\wp} 320)}_{=532}$ is not; $\underbrace{(210 \dot{\wp} 320)}_{=542} \dot{\wp} 100=210 \dot{\wp} \underbrace{(100 \dot{\phi} 320)}_{=431}=553$ are defined but $320 \dot{\wp} \underbrace{(100 \dot{\phi} 210)}_{=321}$ is not; $\underbrace{(210 \dot{\wp} 320)}_{=542} \dot{\wp} 200=320 \dot{\phi} \underbrace{(200 \dot{\wp} 210)}_{=421}=554$ are defined but $210 \dot{\dot{\theta}} \underbrace{(200 \dot{\varnothing} 320)}_{=532}$ is not; $\underbrace{(300 \dot{\phi} 410)}_{=541} \dot{\wp} 100=410 \dot{\wp} \underbrace{(100 \dot{\phi} 300)}_{=410}=552$ are defined but $300 \dot{\phi} \underbrace{(100 \dot{\wp} 410)}_{=521}$ is not; $\underbrace{(300 \dot{\wp} 410)}_{=541} \dot{\varphi} 300=554$ is defined but $410 \dot{\varnothing} \underbrace{(300 \dot{\varphi} 300)}_{=530}$ is not; $\underbrace{(310 \dot{\wp} 310)}_{=542} \dot{\wp} 100=553$ is defined but $310 \dot{\dot{\theta}} \underbrace{(100 \dot{\wp} 310)}_{=421}$ is not; $\underbrace{(310 \dot{\wp} 310)}_{=542} \dot{\varnothing} 200=554$ is defined but $310 \dot{\varnothing} \underbrace{(200 \dot{\wp} 310)}_{=531}$ is not.

From this it follows that the set $D_{5}^{2}$ of all deflected partial binary products within $W_{5}^{\mathcal{N}_{2}}$ is as follows: 
$100 \dot{\wp} 200=310,100 \dot{\wp} 300=410,100 \dot{\wp} 320=431,100 \dot{\wp} 421=532,100 \dot{\varnothing} 541=552,100 \dot{\wp} 542=553,110 \dot{\wp} 200=$ $311,110 \dot{\wp} 220=331,110 \dot{\wp} 300=411,110 \dot{\wp} 311=422,110 \dot{\wp} 330=441,110 \dot{\wp} 331=442,110 \dot{\wp} 411=522$, $110 \dot{\varnothing} 422=533,110 \dot{\wp} 441=552,110 \dot{\varnothing} 442=553,200 \dot{\wp} 210=421,200 \dot{\varnothing} 431=543,200 \dot{\wp} 442=544,200 \dot{\wp} 542=$ $554,210 \dot{\wp} 310=532,210 \dot{\wp} 320=542,210 \dot{\wp} 431=553,220 \dot{\wp} 422=544,300 \dot{\wp} 410=541,300 \dot{\wp} 441=544$, $300 \dot{0} 541=554,310 \dot{\wp} 310=542,310 \dot{\wp} 320=543,311 \dot{\wp} 331=544,320 \dot{\wp} 421=554,330 \dot{\wp} 411=544$ and $410 \dot{\wp} 410=552$.

Now applying $\tilde{s}$ to all steady partial binary products within $\mathcal{W}_{5}^{\mathrm{N}_{2}}$ we obtain the following system of linear equations:

$2 \tilde{s}_{100}=\tilde{s}_{210}, \tilde{s}_{100}+\tilde{s}_{110}=\tilde{s}_{211}, \tilde{s}_{100}+\tilde{s}_{210}=\tilde{s}_{321}, \tilde{s}_{100}+\tilde{s}_{221}=\tilde{s}_{322}, \tilde{s}_{100}+\tilde{s}_{310}=\tilde{s}_{421}, \tilde{s}_{100}+\tilde{s}_{321}=\tilde{s}_{432}$, $\tilde{s}_{100}+\tilde{s}_{332}=\tilde{s}_{433}, \tilde{s}_{100}+\tilde{s}_{400}=\tilde{s}_{510}, \tilde{s}_{100}+\tilde{s}_{410}=\tilde{s}_{521}, \tilde{s}_{100}+\tilde{s}_{420}=\tilde{s}_{531}, \tilde{s}_{100}+\tilde{s}_{430}=\tilde{s}_{541}, \tilde{s}_{100}+\tilde{s}_{431}=\tilde{s}_{542}$, $\tilde{s}_{100}+\tilde{s}_{432}=\tilde{s}_{543}, \tilde{s}_{100}+\tilde{s}_{443}=\tilde{s}_{544}, \tilde{s}_{100}+\tilde{s}_{540}=\tilde{s}_{551}, \tilde{s}_{100}+\tilde{s}_{543}=\tilde{s}_{554}, \tilde{s}_{100}+\tilde{s}_{554}=\tilde{s}_{555}, 2 \tilde{s}_{110}=\tilde{s}_{221}$, $\tilde{s}_{110}+\tilde{s}_{211}=\tilde{s}_{322}, \tilde{s}_{110}+\tilde{s}_{221}=\tilde{s}_{332}, \tilde{s}_{110}+\tilde{s}_{322}=\tilde{s}_{433}, \tilde{s}_{110}+\tilde{s}_{332}=\tilde{s}_{443}, \tilde{s}_{110}+\tilde{s}_{400}=\tilde{s}_{511}, \tilde{s}_{110}+\tilde{s}_{433}=\tilde{s}_{544}$, $\tilde{s}_{110}+\tilde{s}_{440}=\tilde{s}_{551}, \tilde{s}_{110}+\tilde{s}_{443}=\tilde{s}_{554}, \tilde{s}_{110}+\tilde{s}_{544}=\tilde{s}_{555}, 2 \tilde{s}_{111}=\tilde{s}_{222}, \tilde{s}_{111}+\tilde{s}_{222}=\tilde{s}_{333}, \tilde{s}_{111}+\tilde{s}_{333}=\tilde{s}_{444}$, $\tilde{s}_{111}+\tilde{s}_{444}=\tilde{s}_{555}, 2 \tilde{s}_{200}=\tilde{s}_{420}, \tilde{s}_{200}+\tilde{s}_{220}=\tilde{s}_{422}, \tilde{s}_{200}+\tilde{s}_{300}=\tilde{s}_{520}, \tilde{s}_{200}+\tilde{s}_{310}=\tilde{s}_{531}, \tilde{s}_{200}+\tilde{s}_{320}=\tilde{s}_{532}$, $\tilde{s}_{200}+\tilde{s}_{331}=\tilde{s}_{533}, \tilde{s}_{200}+\tilde{s}_{420}=\tilde{s}_{542}, \tilde{s}_{200}+\tilde{s}_{530}=\tilde{s}_{552}, \tilde{s}_{200}+\tilde{s}_{531}=\tilde{s}_{553}, \tilde{s}_{200}+\tilde{s}_{553}=\tilde{s}_{555}, 2 \tilde{s}_{210}=\tilde{s}_{432}$, $\tilde{s}_{210}+\tilde{s}_{300}=\tilde{s}_{521}, \tilde{s}_{210}+\tilde{s}_{321}=\tilde{s}_{543}, \tilde{s}_{210}+\tilde{s}_{430}=\tilde{s}_{552}, \tilde{s}_{210}+\tilde{s}_{432}=\tilde{s}_{554}, \tilde{s}_{210}+\tilde{s}_{543}=\tilde{s}_{555}, \tilde{s}_{211}+\tilde{s}_{221}=\tilde{s}_{433}$, $\tilde{s}_{211}+\tilde{s}_{332}=\tilde{s}_{544}, \tilde{s}_{211}+\tilde{s}_{443}=\tilde{s}_{555}, 2 \tilde{s}_{220}=\tilde{s}_{442}, \tilde{s}_{220}+\tilde{s}_{300}=\tilde{s}_{522}, \tilde{s}_{220}+\tilde{s}_{311}=\tilde{s}_{533}, \tilde{s}_{220}+\tilde{s}_{330}=\tilde{s}_{552}$, $\tilde{s}_{220}+\tilde{s}_{331}=\tilde{s}_{553}, \tilde{s}_{220}+\tilde{s}_{533}=\tilde{s}_{555}, 2 \tilde{s}_{221}=\tilde{s}_{443}, \tilde{s}_{221}+\tilde{s}_{322}=\tilde{s}_{544}, \tilde{s}_{221}+\tilde{s}_{332}=\tilde{s}_{554}, \tilde{s}_{221}+\tilde{s}_{433}=\tilde{s}_{555}$, $2 \tilde{s}_{222}=\tilde{s}_{444}, \tilde{s}_{222}+\tilde{s}_{333}=\tilde{s}_{555}, 2 \tilde{s}_{300}=\tilde{s}_{530}, \tilde{s}_{300}+\tilde{s}_{330}=\tilde{s}_{533}, \tilde{s}_{300}+\tilde{s}_{430}=\tilde{s}_{543}, \tilde{s}_{300}+\tilde{s}_{520}=\tilde{s}_{552}, \tilde{s}_{300}+\tilde{s}_{530}=\tilde{s}_{553}$, $\tilde{s}_{300}+\tilde{s}_{552}=\tilde{s}_{555}, \tilde{s}_{310}+\tilde{s}_{420}=\tilde{s}_{553}, \tilde{s}_{310}+\tilde{s}_{431}=\tilde{s}_{554}, \tilde{s}_{310}+\tilde{s}_{542}=\tilde{s}_{555}, \tilde{s}_{311}+\tilde{s}_{442}=\tilde{s}_{555}, 2 \tilde{s}_{320}=\tilde{s}_{553}$, $\tilde{s}_{320}+\tilde{s}_{532}=\tilde{s}_{555}, 2 \tilde{s}_{321}=\tilde{s}_{554}, \tilde{s}_{321}+\tilde{s}_{432}=\tilde{s}_{555}, \tilde{s}_{322}+\tilde{s}_{332}=\tilde{s}_{555}, \tilde{s}_{330}+\tilde{s}_{522}=\tilde{s}_{555}, \tilde{s}_{331}+\tilde{s}_{422}=\tilde{s}_{555}$, $2 \tilde{s}_{400}=\tilde{s}_{540}, \tilde{s}_{400}+\tilde{s}_{440}=\tilde{s}_{544}, \tilde{s}_{400}+\tilde{s}_{510}=\tilde{s}_{551}, \tilde{s}_{400}+\tilde{s}_{540}=\tilde{s}_{554}, \tilde{s}_{400}+\tilde{s}_{551}=\tilde{s}_{555}, \tilde{s}_{410}+\tilde{s}_{430}=\tilde{s}_{554}$, $\tilde{s}_{410}+\tilde{s}_{541}=\tilde{s}_{555}, \tilde{s}_{411}+\tilde{s}_{441}=\tilde{s}_{555}, 2 \tilde{s}_{420}=\tilde{s}_{554}, \tilde{s}_{420}+\tilde{s}_{531}=\tilde{s}_{555}, \tilde{s}_{421}+\tilde{s}_{431}=\tilde{s}_{555}, \tilde{s}_{430}+\tilde{s}_{521}=\tilde{s}_{555}$, $\tilde{s}_{440}+\tilde{s}_{511}=\tilde{s}_{555}, 2 \tilde{s}_{500}=\tilde{s}_{550}, \tilde{s}_{500}+\tilde{s}_{550}=\tilde{s}_{555}, \tilde{s}_{510}+\tilde{s}_{540}=\tilde{s}_{555}$, and at last $\tilde{s}_{520}+\tilde{s}_{530}=\tilde{s}_{555}$.

Moreover, we have that $\tilde{s}_{000}=s_{0}=0, \tilde{s}_{111}=s_{1}=1 / 5, \tilde{s}_{222}=s_{2}=2 / 5, \tilde{s}_{333}=s_{3}=3 / 5, \tilde{s}_{444}=s_{4}=4 / 5$ and $\tilde{s}_{555}=s_{5}=1$. It appears that our system of linear equations is not complete with $\tilde{s}_{311}$ and $\tilde{s}_{411}$ as parameters $p$ and $q$, respectively, such that $5 / 14 \leq p \leq q \leq 16 / 35, p \leq 3 / 7$ and $q \geq 17 / 42$ (by the isotonicity of $\tilde{s}$ ). To supplement the system, we are looking for consistent equations by applying $\tilde{s}$ to suitable deflected partial binary products. We discover that

(1) the system of linear equations together with equations $\tilde{s}_{110}+\tilde{s}_{311}=\tilde{s}_{422}$ and $\tilde{s}_{110}+\tilde{s}_{330}=\tilde{s}_{441}$,

(2) the system together with equations $\tilde{s}_{110}+\tilde{s}_{311}=\tilde{s}_{422}$ and $\tilde{s}_{110}+\tilde{s}_{300}=\tilde{s}_{411}$ and

(3) the system together with equations $\tilde{s}_{110}+\tilde{s}_{200}=\tilde{s}_{311}$ and $\tilde{s}_{110}+\tilde{s}_{300}=\tilde{s}_{411}$

form three complete systems of linear equations yielding the required solutions. Finally, applying $\tilde{s}$ to all deflected partial binary products, we easily verify remaining assertions of the theorem.

Observe that the 2-extension $\mathcal{W}_{5}^{\mathcal{N}_{2}}$ of the Weber $M V$-chain $\mathcal{W}_{5}$ contains the following four Weber $M V$-chains:

$000<100<100^{2 \dot{\varphi}}(=210)<100^{3 \dot{\varphi}}(=321)<100^{4 \dot{\wp}}(=432)<100^{5 \dot{\varphi}}(=543)<100^{6 \dot{\wp}}(=554)<100^{7 \dot{\varphi}}(=555)$, $000<111<111^{2 \dot{\varphi}}(=222)<111^{3 \dot{\varphi}}(=333)<111^{4 \dot{\varphi}}(=444)<111^{5 \dot{\varphi}}(=555), 000<500<500^{2 \dot{\varphi}}(=550)<$ $500^{3 \dot{\varphi}}(=555)$ and $000<310<310^{2 \dot{\varphi}}(=542)<310^{3 \dot{\varphi}}(=555)$.

We emphasize that PM of $\tilde{s}$ cases to exist on the partial binary product $310 \dot{\wp} 310=542$. This fact opposes an axiom of Weber states.

\section{Proof of Theorem 13}

We have that $W_{2}^{\mathcal{N}_{3}}$ consists of 15 elements: $0000<1000<1100<1110<1111,2000<2100<2110<2111$, $2200<2210<2211,2220<2221<2222$. An ordinary calculation shows that the set $E_{2}^{3}$ of all possible existing partial binary products within $\mathcal{W}_{2}^{\mathcal{N}_{3}}$ is as follows:

$1000 \dot{\wp} 1000=2100,1000 \dot{\wp} 1100=2110,1000 \dot{\wp} 1110=2111,1000 \dot{\wp} 2100=2210,1000 \dot{\varnothing} 2110=2211$, $1000 \dot{\wp} 2210=2221,1000 \dot{\wp} 2221=2222,1100 \dot{\wp} 1100=2211,1100 \dot{\wp} 2110=2221,1100 \dot{\wp} 2211=2222$, $1110 \dot{\wp} 1110=2221,1110 \dot{\wp} 2111=2222,1111 \dot{\wp} 1111=2222,2000 \dot{\varnothing} 2000=2200,2000 \dot{\wp} 2200=2220$, $2000 \dot{\wp} 2220=2222,2100 \dot{\wp} 2100=2221,2100 \dot{\varnothing} 2210=2222,2110 \dot{\varnothing} 2110=2222,2200 \dot{\wp} 2200=2222$ and $x \dot{0} 0000=x$ for all $x \in \mathcal{W}_{2}^{\mathcal{N}_{3}}$. 
Now verifying each of the partial triple products in turn, we establish the next two exclusive situations:

$$
\begin{aligned}
& \underbrace{(1000 \dot{\wp} 1100)}_{=2110} \dot{\wp} 1000=2211 \text { is defined but } 1100 \dot{\wp} \underbrace{(1000 \dot{\wp} 1000)}_{=2100} \text { is not and } \\
& \underbrace{(1000 \dot{\wp} 1100)}_{=2110} \dot{\wp} 1100=2221 \text { is defined but } 1000 \wp \dot{\varphi} \underbrace{(1100 \dot{\wp} 1100)}_{=2211} \text { is not. }
\end{aligned}
$$

From this it follows that the set $D_{2}^{3}$ of all deflected binary partial products within $\mathcal{W}_{2}^{\mathcal{N}_{3}}$ consists of three members: $1000 \dot{\wp} 1100=2110,1000 \dot{\wp} 2110=2211$ and $1100 \dot{\wp} 2110=2221$.

Now applying $\tilde{s}$ to all steady binary partial products within $\mathcal{W}_{2}^{N_{3}}$, we obtain the following system of linear equations:

$2 \tilde{s}_{1000}=\tilde{s}_{2100}, \tilde{s}_{1000}+\tilde{s}_{1110}=\tilde{s}_{2111}, \tilde{s}_{1000}+\tilde{s}_{2100}=\tilde{s}_{2210}, \tilde{s}_{1000}+\tilde{s}_{2210}=\tilde{s}_{2221}, \tilde{s}_{1000}+\tilde{s}_{2221}=\tilde{s}_{2222}, 2 \tilde{s}_{1100}=\tilde{s}_{2211}$, $\tilde{s}_{1100}+\tilde{s}_{2211}=\tilde{s}_{2222}, 2 \tilde{s}_{1110}=\tilde{s}_{2221}, \tilde{s}_{1110}+\tilde{s}_{2111}=\tilde{s}_{2222}, 2 \tilde{s}_{1111}=\tilde{s}_{2222}, 2 \tilde{s}_{20000}=\tilde{s}_{2200}, \tilde{s}_{2000}+\tilde{s}_{2200}=\tilde{s}_{2220}$, $\tilde{s}_{2000}+\tilde{s}_{2220}=\tilde{s}_{2222}, 2 \tilde{s}_{2100}=\tilde{s}_{2221}, \tilde{s}_{2100}+\tilde{s}_{2210}=\tilde{s}_{2222}, 2 \tilde{s}_{2110}=\tilde{s}_{2222}$ and $2 \tilde{s}_{2200}=\tilde{s}_{2222}$.

In view of the extension property: $\tilde{s}_{0000}=s_{0}=0, \tilde{s}_{1111}=s_{1}=1 / 2$ and $\tilde{s}_{2222}=s_{2}=1$, we obtain solutions, as asserted.

Remark that the 3-extension $\mathcal{W}_{2}^{\mathcal{N}_{3}}$ of the Weber $M V$-chain $\mathcal{W}_{2}$ contains the following six Weber $M V$-chains:

$0000<1000<1000^{2 \dot{\beta}}(=2100)<1000^{3 \dot{\phi}}(=2210)<1000^{4 \dot{\varphi}}(=2221)<1000^{5 \dot{\varphi}}(=2222), 0000<1100<$ $1100^{2 \dot{\phi}}(=2211)<1100^{3 \dot{\phi}}(=2222), 0000<1111<1111^{2 \dot{\phi}}(=2222), 0000<2000<2000^{2 \dot{\varphi}}(=2200)<$ $2000^{3 \dot{\hat{\rho}}}(=2220)<2000^{4 \dot{\boldsymbol{\rho}}}(=2222), 0000<2110<2110^{2 \dot{\boldsymbol{\rho}}}(=2222)$ and $0000<2200<2200^{2 \dot{\boldsymbol{\rho}}}(=2222)$.

\section{Proof of Theorem 14}

We have that $W_{3}^{N_{3}}$ consists of 35 elements: $0000<1000<1100<1110<1111,2000<2100<2110<2111$, $2200<2210<2211,2220<2221<2222,3000<3100<3110<3111,3200<3210<3211,3220<3221<$ $3222,3300<3310<3311,3320<3321<3322,3330<3331<3332<3333$. A routine calculation shows that the set $E_{3}^{3}$ of all possible existing partial binary products within $W_{3}^{N_{3}}$ is as follows:

$1000 \dot{\wp} 1000=2100,1000 \dot{\wp} 1100=2110,1000 \dot{\wp} 1110=2111,1000 \dot{\wp} 2000=3100,1000 \dot{\wp} 2100=3210$, $1000 \dot{8} 2110=3211,1000 \dot{\wp} 2210=3221,1000 \dot{\wp} 2221=3222,1000 \dot{\wp} 3200=3310,1000 \dot{\wp} 3210=3321$, $1000 \dot{\wp} 3221=3322,1000 \dot{\wp} 3320=3331,1000 \dot{\wp} 3321=3332,1000 \dot{\wp} 3332=3333,1100 \dot{\wp} 1100=2211$, $1100 \dot{\wp} 2000=3110,1100 \dot{\wp} 2100=3211,1100 \dot{\wp} 2110=3221,1100 \dot{\wp} 2200=3311,1100 \dot{\varnothing} 2210=3321$,

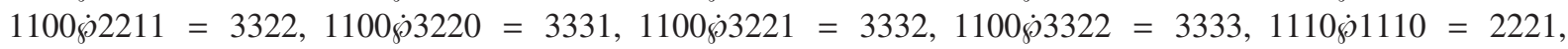
$1110 \dot{\wp} 2000=3111,1110 \dot{\wp} 2111=3222,1110 \dot{\wp} 2220=3331,1110 \dot{\wp} 2221=3332,1110 \dot{\wp} 3222=3333$, $1111 \dot{\wp} 1111=2222,1111 \dot{\wp} 2222=3333,2000 \dot{\wp} 2000=3200,2000 \wp 2200=3220,2000 \dot{\wp} 2220=3222$,

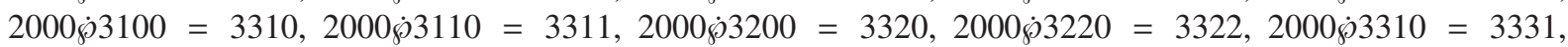
$2000 \dot{\wp} 3320=3332,2000 \dot{\wp} 3331=3333,2100 \dot{\wp} 2100=3321,2100 \dot{\wp} 2210=3322,2100 \dot{\wp} 3210=3332$, $2100 \dot{\wp} 3321=3333,2110 \dot{\wp} 2110=3322,2110 \dot{\wp} 2210=3332,2110 \dot{\wp} 3221=3333,2111 \dot{\wp} 2221=3333$, $2200 \dot{\wp} 2200=3322,2200 \dot{\wp} 3110=3331,2200 \dot{\wp} 3311=3333,2210 \dot{\wp} 3211=3333,2211 \dot{\wp} 2211=3333$, $2220 \dot{\wp} 3111=3333,3000 \dot{\wp} 3000=3300,3000 \dot{\wp} 3300=3330,3000 \wp 3330=3333,3100 \dot{\wp} 3200=3331$, $3100 \dot{\wp} 3320=3333,3110 \dot{\wp} 3220=3333,3200 \dot{\wp} 3200=3332,3200 \dot{\wp} 3310=3333,3210 \dot{\wp} 3210=3333$, $3300 \dot{\wp} 3300=3333$ and $x \dot{\wp} 0000=x$ for all $x \in \mathcal{W}_{3}^{\mathcal{N}_{3}}$.

Now verifying each of the partial triple products in turn, we establish the following eight "non-associative" situations:

$\underbrace{(1000 \dot{\wp} 1100)}_{=2110} \dot{\wp} 1100=3221$ is defined but $1000 \dot{\wp} \underbrace{(1100 \dot{\wp} 1100)}_{2211}$ is not;

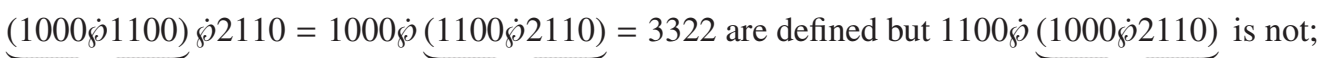

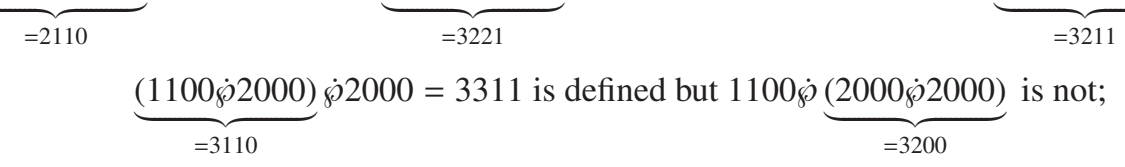

$\underbrace{(1100 \dot{\wp} 2000)}_{=3110} \dot{\wp} 2200=1100 \dot{\wp} \underbrace{(2000 \dot{\wp} 2200)}_{=3220}=3331$ are defined but $2000 \dot{\wp} \underbrace{(1100 \dot{\wp} 2200)}_{=3311}$ is not; 
$\underbrace{(1100 \dot{\wp} 2110)}_{=3221} \dot{\wp} 1000=2110 \dot{\wp} \underbrace{(1000 \dot{\wp} 1100)}_{=2110}=3322$ are defined but $1100 \dot{\wp} \underbrace{(1000 \dot{\wp} 2110)}_{=3211}$ is not;

$\underbrace{(1100 \dot{8} 2110)}_{=3221} \dot{\wp} 1100=3332$ is defined but $2110 \dot{\wp} \underbrace{(1100 \wp 1100)}_{=2211}$ is not;

$\underbrace{(2000 \dot{\wp} 2200)}_{=3220} \dot{\wp} 1100=2200 \dot{\wp} \underbrace{(1100 \dot{\wp} 2000)}_{=3110}=3331$ are defined but $2000 \dot{\wp} \underbrace{(1100 \dot{8} 2200)}_{=3311}$ is not;

$\underbrace{(2000 \dot{8} 2200)}_{=3220} \dot{\wp} 2000=3322$ is defined but $2200 \dot{\wp} \underbrace{(2000 \dot{\wp} 2000)}_{=3200}$ is not.

From this it follows that the set $D_{3}^{3}$ of all deflected binary partial products within $\mathcal{W}_{3}^{\mathcal{N}_{3}}$ is the following:

$1000 \dot{\wp} 1100=2110,1000 \dot{\wp} 3221=3322,1100 \dot{\wp} 2000=3110,1100 \dot{\wp} 2110=3221,1100 \dot{\wp} 3220=3331$, $1100 \dot{\wp} 3221=3332,2000 \wp 2200=3220,2000 \dot{\wp} 3110=3311,2000 \dot{\wp} 3220=3322,2110 \dot{\wp} 2110=3322$ and $2200 \dot{\beta} 3110=3331$.

Now applying $\tilde{s}$ to $S_{3}^{3}$ within $\mathcal{W}_{3}^{\mathcal{N}_{3}}$, we obtain the following system of linear equations:

$2 \tilde{s}_{1000}=\tilde{s}_{2100}, \tilde{s}_{1000}+\tilde{s}_{1110}=\tilde{s}_{2111}, \tilde{s}_{1000}+\tilde{s}_{2000}=\tilde{s}_{3100}, \tilde{s}_{1000}+\tilde{s}_{2100}=\tilde{s}_{3210}, \tilde{s}_{1000}+\tilde{s}_{2110}=\tilde{s}_{3211}, \tilde{s}_{1000}+\tilde{s}_{2210}=\tilde{s}_{3221}$, $\tilde{s}_{1000}+\tilde{s}_{2221}=\tilde{s}_{3222}, \tilde{s}_{1000}+\tilde{s}_{3200}=\tilde{s}_{3310}, \tilde{s}_{1000}+\tilde{s}_{3210}=\tilde{s}_{3321}, \tilde{s}_{1000}+\tilde{s}_{3320}=\tilde{s}_{3331}, \tilde{s}_{1000}+\tilde{s}_{3321}=\tilde{s}_{3332}$, $\tilde{s}_{1000}+\tilde{s}_{3332}=\tilde{s}_{3333}, 2 \tilde{s}_{1100}=\tilde{s}_{2211}, \tilde{s}_{1100}+\tilde{s}_{2100}=\tilde{s}_{3211}, \tilde{s}_{1100}+\tilde{s}_{2200}=\tilde{s}_{3311}, \tilde{s}_{1100}+\tilde{s}_{2210}=\tilde{s}_{3321}, \tilde{s}_{1100}+\tilde{s}_{2211}=\tilde{s}_{3322}$, $\tilde{s}_{1100}+\tilde{s}_{3322}=\tilde{s}_{3333}, 2 \tilde{s}_{1110}=\tilde{s}_{2221}, \tilde{s}_{1110}+\tilde{s}_{2000}=\tilde{s}_{3111}, \tilde{s}_{1110}+\tilde{s}_{2111}=\tilde{s}_{3222}, \tilde{s}_{1110}+\tilde{s}_{2220}=\tilde{s}_{3331}, \tilde{s}_{1110}+\tilde{s}_{2221}=\tilde{s}_{3332}$, $\tilde{s}_{1110}+\tilde{s}_{3222}=\tilde{s}_{3333}, 2 \tilde{s}_{1111}=\tilde{s}_{2222}, \tilde{s}_{1111}+\tilde{s}_{2222}=\tilde{s}_{3333}, 2 \tilde{s}_{2000}=\tilde{s}_{3200}, \tilde{s}_{2000}+\tilde{s}_{2220}=\tilde{s}_{3222}, \tilde{s}_{2000}+\tilde{s}_{3100}=\tilde{s}_{3310}$, $\tilde{s}_{2000}+\tilde{s}_{3200}=\tilde{s}_{3320}, \tilde{s}_{2000}+\tilde{s}_{3310}=\tilde{s}_{3331}, \tilde{s}_{2000}+\tilde{s}_{3320}=\tilde{s}_{3332}, \tilde{s}_{2000}+\tilde{s}_{3331}=\tilde{s}_{3333}, 2 \tilde{s}_{2100}=\tilde{s}_{3321}, \tilde{s}_{2100}+\tilde{s}_{2210}=\tilde{s}_{3322}$, $\tilde{s}_{2100}+\tilde{s}_{3210}=\tilde{s}_{3332}, \tilde{s}_{2100}+\tilde{s}_{3321}=\tilde{s}_{3333}, \tilde{s}_{2110}+\tilde{s}_{2210}=\tilde{s}_{3332}, \tilde{s}_{2110}+\tilde{s}_{3221}=\tilde{s}_{3333}, \tilde{s}_{2111}+\tilde{s}_{2221}=\tilde{s}_{3333}$, $2 \tilde{s}_{2200}=\tilde{s}_{3322}, \tilde{s}_{2200}+\tilde{s}_{3311}=\tilde{s}_{3333}, \tilde{s}_{2210}+\tilde{s}_{3211}=\tilde{s}_{3333}, 2 \tilde{s}_{2211}=\tilde{s}_{3333}, \tilde{s}_{2220}+\tilde{s}_{3111}=\tilde{s}_{3333}, 2 \tilde{s}_{3000}=\tilde{s}_{3300}$, $\tilde{s}_{3000}+\tilde{s}_{3300}=\tilde{s}_{3330}, \tilde{s}_{3000}+\tilde{s}_{3330}=\tilde{s}_{3333}, \tilde{s}_{3100}+\tilde{s}_{3200}=\tilde{s}_{3331}, \tilde{s}_{3100}+\tilde{s}_{3320}=\tilde{s}_{3333}, \tilde{s}_{3110}+\tilde{s}_{3220}=\tilde{s}_{3333}$, $2 \tilde{s}_{3200}=\tilde{s}_{3332}, \tilde{s}_{3200}+\tilde{s}_{3310}=\tilde{s}_{3333}, 2 \tilde{s}_{3210}=\tilde{s}_{3333}$ and $2 \tilde{s}_{3300}=\tilde{s}_{3333}$.

Moreover, we have that $\tilde{s}_{0000}=s_{0}=0, \tilde{s}_{1111}=s_{1}=1 / 3, \tilde{s}_{2222}=s_{2}=2 / 3$ and $\tilde{s}_{3333}=s_{3}=1$. It appears that this system is not complete with $\tilde{s}_{3110}$ as a parameter $p$ such that $5 / 12 \leq p \leq 35 / 72$. To supplement the system, we are looking for a consistent equation by applying $\tilde{s}$ to suitable deflected partial products. We choose the next complementary equation:

$$
\tilde{s}_{2000}+\tilde{s}_{3110}=\tilde{s}_{3311}
$$

Finally, the completed system yield the required solutions. In view of these solutions, we apply $\tilde{s}$ to all deflected products in order to verify remaining assertions of the theorem.

Note that the 3-extension $\mathcal{W}_{3}^{N_{3}}$ of the Weber $M V$-chain $\mathcal{W}_{3}$ contains the following six Weber $M V$-chains:

$0000<1000<1000^{2 \dot{\varphi}}(=2100)<1000^{3 \dot{\varphi}}(=3210)<1000^{4 \dot{\varphi}}(=3321)<1000^{5 \dot{\phi}}(=3332)<1000^{6 \dot{\xi}}(=3333)$, $0000<1100<1100^{2 \dot{\varphi}}(=2211)<1100^{3 \dot{\phi}}(=3322)<1100^{4 \dot{\varphi}}(=3333), 0000<1111<1111^{2 \dot{\phi}}(=2222)<$ $1111^{3 \dot{\phi}}(=3333), 0000<2211<2211^{2 \dot{\phi}}(=3333), 0000<3000<3000^{2 \dot{\phi}}(=3300)<3000^{3 \dot{\varphi}}(=3330)<$ $3000^{4 \dot{\varphi}}(=3333)$ and $0000<3210<3210^{2 \dot{\varphi}}(=3333)$.

\section{Proof of Theorem 15}

We have that $\mathcal{W}_{4}^{N_{3}}$ consists of 70 elements: $0000<1000<1100<1110<1111,2000<2100<2110<$ 2111, $2200<2210<2211,2220<2221<2222,3000<3100<3110<3111,3200<3210<3211$, $3220<3221<3222,3300<3310<3311,3320<3321<3322,3330<3331<3332<3333,4000<$ $4100<4110<4111,4200<4210<4211,4220<4221<4222,4300<4310<4311,4320<4321<4322$, $4330<4331<4332<4333,4400<4410<4411,4420<4421<4422,4430<4431<4432<4433$, $4440<4441<4442<4443<4444$.

A routine calculation shows that the set $E_{4}^{3}$ of all existing partial binary products within $\mathcal{W}_{4}^{\mathrm{N}_{3}}$ are as follows:

$1000 \dot{\wp} 1000=2100,1000 \dot{\wp} 1100=2110,1000 \dot{\wp} 1110=2111,1000 \dot{\wp} 2000=3100,1000 \dot{\wp} 2100=3210$, $1000 \dot{\wp} 2110=3211,1000 \dot{8} 2210=3221,1000 \dot{\wp} 2221=3222,1000 \dot{\wp} 3000=4100,1000 \dot{\wp} 3100=4210$, $1000 \dot{\wp} 3110=4211,1000 \dot{\wp} 3200=4310,1000 \dot{\wp} 3210=4321,1000 \wp 3221=4322,1000 \dot{\wp} 3320=4331$, $1000 \dot{\wp 3} 321=4332,1000 \dot{\wp} 3332=4333,1000 \dot{\wp} 4300=4410,1000 \wp 4310=4421,1000 \dot{6} 4320=4431$, $1000 \dot{\varphi} 4321=4432,1000 \dot{\wp} 4332=4433,1000 \dot{\wp} 4430=4441,1000 \wp 4431=4442,1000 \dot{6} 4432=4443$, $1000 \dot{\wp} 4443=4444,1100 \dot{\wp} 1100=2211,1100 \dot{\wp} 2000=3110,1100 \wp 2100=3211,1100 \dot{\wp} 2110=3221$, 
$1100 \dot{\varnothing} 2200=3311,1100 \dot{\varnothing} 2210=3321,1100 \dot{\varnothing} 2211=3322,1100 \dot{\wp} 3000=4110,1100 \dot{\varnothing} 3100=4211$, $1100 \dot{\phi} 3110=4221,1100 \dot{\wp} 3200=4311,1100 \dot{\wp} 3211=4322,1100 \dot{\wp} 3220=4331,1100 \dot{\wp} 3221=4332$, $1100 \dot{\varnothing} 3300=4411,1100 \dot{\wp} 3310=4421,1100 \dot{\ominus} 3311=4422,1100 \dot{\wp} 3320=4431,1100 \dot{\wp} 3321=4432$, $1100 \dot{\phi} 3322=4433,1100 \dot{\varphi} 4330=4441,1100 \dot{\varphi} 4331=4442,1100 \dot{\varphi} 4332=4443,1100 \dot{\varphi} 4433=4444$, $1110 \dot{\wp} 1110=2221,1110 \dot{\wp} 2000=3111,1110 \dot{\wp} 2100=3211,1110 \dot{\varnothing} 2111=3222,1110 \dot{\varnothing} 2220=3331$, $1110 \dot{\phi} 2221=3332,1110 \dot{\wp} 3000=4111,1110 \dot{\wp} 3111=4222,1110 \dot{\wp} 3222=4333,1110 \dot{\wp} 3330=4441$, $1110 \dot{\varnothing} 3331=4442,1110 \dot{\wp} 3332=4443,1110 \dot{\wp} 4333=4444,2000 \dot{\wp} 2000=4200,2000 \dot{\wp} 2100=4210$, $2000 \dot{\varnothing} 2110=4211,2000 \dot{\wp} 2200=4220,2000 \dot{\varphi} 2210=4221,2000 \dot{\varphi} 2220=4222,2000 \dot{\wp} 3100=4310$, $2000 \dot{\varnothing} 3110=4311,2000 \dot{\wp} 3200=4320,2000 \dot{8} 3220=4322,2000 \dot{\phi} 3310=4331,2000 \dot{\varnothing} 3320=4332$,

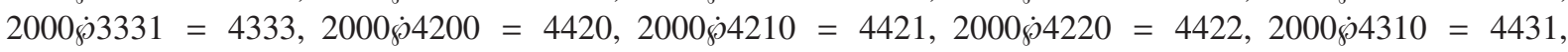
$2000 \dot{\varnothing} 4320=4432,2000 \dot{\wp} 4331=4433,2000 \dot{\wp} 4420=4442,2000 \dot{\rho} 4431=4443,2000 \dot{\wp} 4442=4444$,

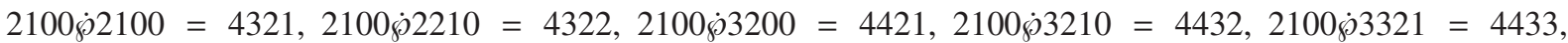
$2100 \dot{\varnothing} 4320=4442,2100 \dot{\varnothing} 4321=4443,2100 \dot{\varphi} 4432=4444,2110 \dot{\phi} 2110=4322,2110 \dot{\varnothing} 2210=4332$,

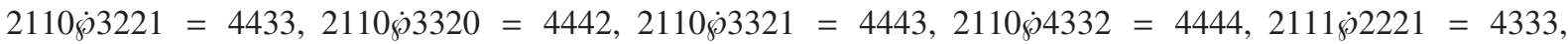
$2111 \dot{\wp} 3332=4444,2200 \dot{\wp} 2200=4422,2200 \dot{\wp} 3110=4331,2200 \dot{\wp} 3311=4433,2200 \dot{\wp} 4220=4442$, $2200 \dot{\varnothing} 4422=4444,2210 \dot{\wp} 2210=4432,2210 \dot{\wp} 3211=4433,2210 \dot{\wp} 3220=4442,2210 \dot{\wp} 3221=4443$, $2210 \dot{\wp} 4322=4444,2211 \dot{\wp} 2211=4433,2211 \dot{\wp} 3322=4444,2220 \dot{\varnothing} 2220=4442,2220 \dot{\wp} 3111=4333$, $2220 \dot{\varnothing} 4222=4444,2221 \dot{\wp} 2221=4443,2221 \dot{\wp} 3222=4444,3000 \dot{\wp} 3000=4300,3000 \dot{\wp} 3300=4330$, $3000 \dot{\wp} 3330=4333,3000 \dot{\wp} 4100=4410,3000 \dot{\rho} 4110=4411,3000 \dot{\wp} 4300=4430,3000 \dot{\wp} 4330=4433$,

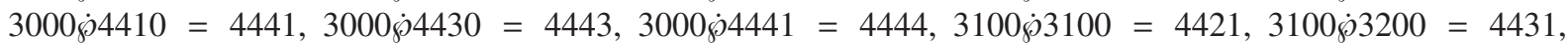

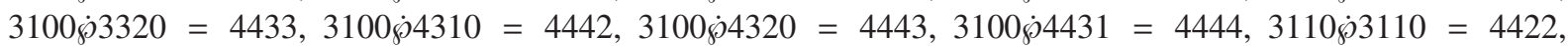
$3110 \dot{\wp} 3220=4433,3110 \dot{\wp} 3310=4442,3110 \dot{\wp} 3320=4443,3110 \dot{\wp} 4331=4444,3111 \dot{\wp} 3331=4444$, $3200 \dot{\wp} 3200=4432,3200 \dot{\wp} 3310=4433,3200 \dot{\varphi} 4210=4442,3200 \dot{\varphi} 4310=4443,3200 \dot{\varphi} 4421=4444$, $3210 \dot{\wp} 3210=4443,3210 \dot{\wp} 4321=4444,3211 \dot{\wp} 3321=4444,3220 \dot{\wp} 4221=4444,3221 \dot{\wp} 3221=4444$, $3300 \dot{\wp} 3300=4433,3300 \dot{\wp} 4110=4441,3300 \dot{\wp} 4411=4444,3310 \dot{8} 4311=4444,3311 \dot{\wp} 3311=4444$,

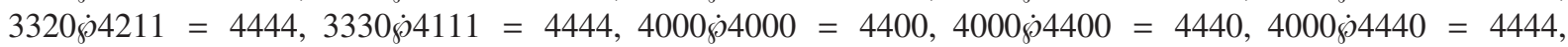
$4100 \dot{\wp} 4300=4441,4100 \dot{\wp} 4430=4444,4110 \dot{\wp} 4330=4444,4200 \dot{\wp} 4200=4442,4200 \dot{\wp} 4420=4444$, $4210 \dot{\wp} 4320=4444,4220 \dot{\wp} 4220=4444,4300 \wp 4300=4443,4300 \dot{\wp} 4410=4444,4310 \dot{\wp} 4310=4444$, $4400 \dot{\wp} 4400=4444$ and $x \dot{\wp} 0000=x$ for all $x \in \mathcal{W}_{4}^{\mathcal{N}_{3}}$.

Now verifying each of the partial triple products in turn, we establish the following forty one exclusive situations:

$\underbrace{(1000 \dot{\wp} 1100)}_{=2110} \dot{\wp} 1100=3221$ is defined but $1000 \dot{\wp} \underbrace{(1100 \dot{\wp} 1100)}_{=2211}$ is not;

$\underbrace{(1000 \dot{\wp} 1100)}_{=2110} \dot{\wp} 3221=1000 \dot{\wp} \underbrace{(1100 \dot{\phi} 3221)}_{=4332}=4433$ are defined but $1100 \dot{\wp} \underbrace{(1000 \dot{\wp} 3221)}_{=4322}$ is not;

$\underbrace{(1000 \dot{\wp} 2000)}_{=3100} \dot{\gamma} 2000=4310$ is defined but $1000 \dot{\wp} \underbrace{(2000 \dot{\wp} 2000)}_{=4200}$ is not;

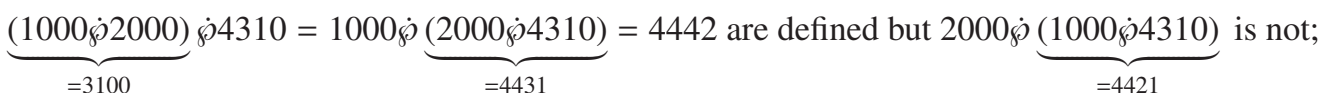

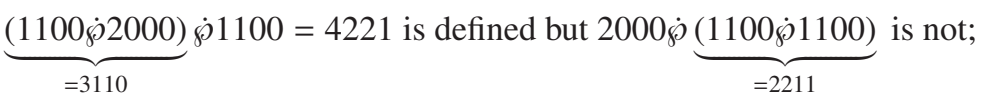

$\underbrace{(1100 \dot{\varnothing} 2000)}_{=3110} \dot{\wp} 2000=4311$ is defined but $1100 \dot{\wp} \underbrace{(2000 \dot{\wp} 2000)}_{=4200}$ is not;

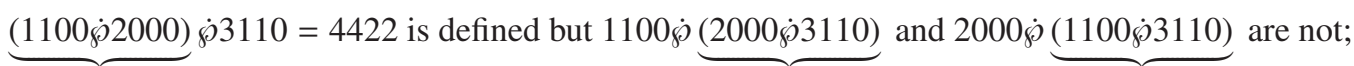

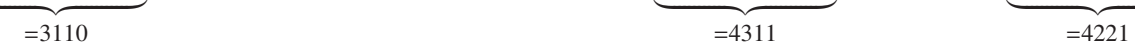

$\underbrace{(1100 \dot{\varnothing} 2000)}_{=3110} \dot{\dot{\theta}} 3220=2000 \dot{\wp} \underbrace{(1100 \dot{\theta} 3220)}_{=4331}=4433$ are defined but $1100 \dot{\varnothing} \underbrace{(2000 \dot{\theta} 3220)}_{=4322}$ is not;

$\underbrace{(1100 \dot{8} 2000)}_{=3110} \dot{\dot{\theta}} 3310=1100 \dot{\oint} \underbrace{(2000 \dot{\phi} 3310)}_{=4331}=4442$ are defined but $2000 \dot{\varnothing} \underbrace{(1100 \dot{\theta} 3310)}_{=4421}$ is not;

$\underbrace{(1100 \dot{\wp} 2100)} \dot{\wp} 1100=4322$ is defined but $2100 \dot{\wp}(1100 \dot{\wp} 1100)$ is not;

$=3211 \quad \underbrace{1100 \varphi 1100)}_{=2211}$ 
$\underbrace{(1100 \dot{\wp} 2100)}_{=3211} \dot{\wp} 2210=2100 \dot{\wp} \underbrace{(1100 \dot{\wp} 2210)}_{=3321}=4433$ are defined but $1100 \dot{\wp} \underbrace{(2100 \dot{8} 2210)}_{=4322}$ is not;

$\underbrace{\wp}_{=3221100 \dot{8} 2110)} \dot{1} 100=4332$ is defined but $2110 \dot{\wp}(\underbrace{(1100 \dot{\wp} 1100)}$ is not;

$=3221$

$=2211$

$\underbrace{(1100 \dot{0} 2110)} \dot{\wp} 2110=4433$ is defined but $1100 \wp \dot{(2110 \dot{8} 2110)}$ is not;

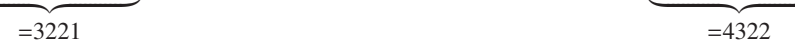

$\underbrace{(1100 \dot{8} 2200)}_{=3311} \dot{\wp} 1100=4422$ is defined but $2200 \dot{\wp} \underbrace{(1100 \dot{\wp} 1100)}_{=2211}$ is not;

$\underbrace{(1100 \dot{\wp} 2200)}_{=3311} \dot{\wp} 2200=4433$ is defined but $1100 \dot{\wp} \underbrace{(2200 \dot{\wp} 2200)}_{=4422}$ is not;

$\underbrace{(1100 \dot{\wp} 2210)}_{=3321} \dot{\wp} 1100=4432$ is defined but $2210 \dot{\wp} \underbrace{(1100 \dot{\wp} 1100)}_{=2211}$ is not;

$\underbrace{(1100 \dot{\wp} 2210)}_{=3321} \dot{\dot{2}} 2100=2210 \dot{\wp} \underbrace{(1100 \dot{\phi} 2100)}_{=3211}=4433$ are defined but $1100 \dot{\wp} \underbrace{(2100 \dot{8} 2210)}_{=4322}$ is not;

$\underbrace{(1100 \dot{\wp} 3000)}_{=4110} \dot{\wp} 3000=4411$ is defined but $1100 \dot{\wp} \underbrace{(3000 \dot{\wp} 3000)}_{=4300}$ is not;

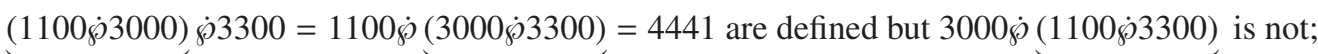

$=4110 \quad \underbrace{3000 \dot{3} 300}_{=4330}=4441$ a defing

$\underbrace{(1100 \dot{\beta} 3220)}_{=4331} \dot{\wp} 1100=4442$ is defined but $3220 \dot{\wp} \underbrace{(1100 \dot{\wp} 1100)}_{=2211}$ is not;

$\underbrace{(1100 \dot{\wp} 3220)}_{=4331} \dot{\wp} 2000=3220 \dot{\wp} \underbrace{(1100 \dot{\wp} 2000)}_{=3110}=4433$ are defined but $1100 \dot{\wp} \underbrace{(2000 \dot{8} 3220)}_{=4322}$ is not;

$\underbrace{(1100 \dot{\wp} 3221)}_{=4332} \dot{\wp} 1000=3221 \dot{\wp} \underbrace{(1000 \dot{\wp} 1100)}_{=2110}=4433$ are defined but $1100 \dot{\wp} \underbrace{(1000 \dot{\wp} 3221)}_{=4322}$ is not;

$\underbrace{(1100 \dot{\wp} 3221)}_{=4332} \dot{\wp} 1100=4443$ is defined but $3221 \dot{\wp} \underbrace{(1100 \dot{\wp} 1100)}_{=2211}$ is not;

$\underbrace{(1110 \dot{\wp} 2000)}_{=3111} \dot{\wp} 1110=4222$ is defined but $2000 \dot{\wp} \underbrace{(1110 \dot{\wp} 1110)}_{=2221}$ is not;

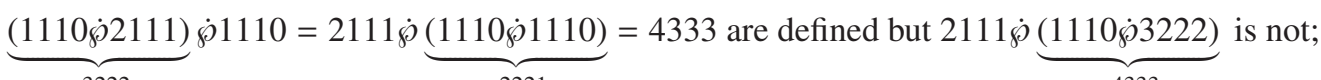

$=3222 \quad \underbrace{1110 \dot{1110}}_{=2221}=4333$ red

$\underbrace{(1110 \dot{\wp} 2220)}_{=3331} \dot{\wp} 1110=4442$ is defined but $2220 \dot{\wp} \underbrace{(1110 \dot{\wp} 1110)}_{=2221}$ is not;

$\underbrace{(1110 \dot{\wp} 220)}_{=333} \dot{\wp} 2000=4333$ and $2220 \dot{\wp}(1110 \dot{\wp} 2000)$ are defined but $1110 \dot{\wp} \underbrace{(22)}_{=422000 \dot{2} 220)}$ is not;

$=3331 \quad \underbrace{20002220}_{=4222}$

$\underbrace{(2000 \dot{\wp} 2100)}_{=4210} \dot{\wp} 2000=4421$ is defined but $2100 \dot{\wp} \underbrace{(2000 \dot{\wp} 2000)}_{=4200}$ is not;

$\underbrace{(2000 \dot{\wp} 2100)}_{=4210} \dot{\beta} 3200=2100 \dot{\wp} \underbrace{(2000 \dot{8} 3200)}_{=4320}=4442$ are defined but $2000 \dot{\wp} \underbrace{(2100 \dot{8} 3200)}_{=4421}$ is not;

$\underbrace{(2000 \dot{\wp} 2200)}_{=4220} \dot{\wp} 2000=4422$ is defined but $2200 \dot{\wp} \underbrace{(2000 \dot{\wp} 2000)}_{=4200}$ is not;

$\underbrace{(2000 \dot{\wp} 2200)}_{=4220} \dot{\wp} 2200=4442$ is defined but $2000 \dot{\oint} \underbrace{(2200 \dot{\wp} 2200)}_{=4422}$ is not;

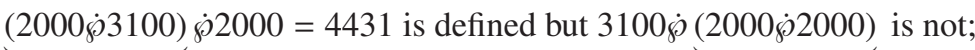

$$
=4310
$$

$=4200$ 


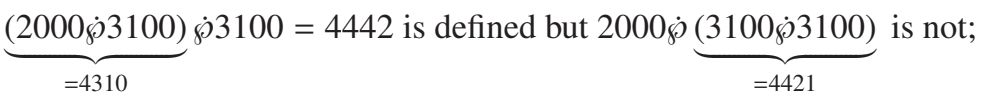

$\underbrace{(2000 \dot{\beta} 3200)}_{=4320} \dot{\wp} 2000=4432$ is defined but $3200 \dot{\phi} \underbrace{(2000 \dot{\wp} 2000)}_{=4200}$ is not;

$\underbrace{(2000 \dot{\wp} 3310)}_{=4331} \dot{\wp} 1100=3310 \dot{\wp} \underbrace{(1100 \dot{\phi} 2000)}_{=3110}=4442$ are defined but $2000 \dot{\phi} \underbrace{(1100 \dot{\wp} 3310)}_{=4421}$ is not;

$\underbrace{(2000 \dot{\varnothing} 3310)}_{=4331} \dot{8} 2000=4433$ is defined but $3310 \dot{\wp} \underbrace{(2000 \dot{8} 2000)}_{=4200}$ is not;

$\underbrace{(2000 \dot{\varnothing} 4310)}_{=4431} \dot{\wp} 1000=4310 \dot{\wp} \underbrace{(1000 \dot{8} 2000)}_{=3100}=4442$ are defined but $2000 \dot{\varnothing} \underbrace{(1000 \dot{8} 4310)}_{=4421}$ is not;

$\underbrace{(2000 \dot{\gamma} 4310)}_{=4431} \dot{\wp} 2000=4443$ is defined but $4310 \dot{\wp} \underbrace{(2000 \dot{\wp} 2000)}_{=4200}$ is not;

$\underbrace{(2200 \dot{\wp} 3110)}_{=4331} \dot{\varphi} 2000=4333$ is defined but $2200 \dot{\oint} \underbrace{(2000 \dot{\varphi} 3110)}_{=4311}$ and $3110 \dot{\varnothing} \underbrace{(2000 \dot{8} 2200)}_{=4220}$ are not;

$\underbrace{(3000 \dot{\wp} 3300)} \dot{\wp} 1100=3300 \dot{\wp}(1100 \dot{\varnothing} 3000)=4441$ are defined but $3000 \dot{\wp}(1100 \dot{\varnothing} 3300)$ is not;

$=4330 \quad \underbrace{1100 \dot{\beta} 3000)}_{=4110} 4441$ a dis

$\underbrace{(3000 \dot{\wp} 3300)} \dot{\wp} 3000=4433$ is defined but $3300 \dot{\wp} \underbrace{(3000 \dot{\varphi} 3000)}$ is not.

$=4330 \quad \underbrace{3000}_{=4300}$

Now gathering all partial binary products from existing partial triple products, we form the set $D_{4}^{3}$ within $\mathcal{W}_{4}^{\mathcal{N}_{3}}$; it consists of the following deflected partial binary products:

$1000 \dot{\wp} 1100=2110,1000 \dot{\wp} 2000=3100,1000 \dot{\wp} 4332=4433,1000 \dot{\wp} 4431=4442,1100 \dot{\wp} 2000=3110$, $1100 \dot{\phi} 2100=3211,1100 \dot{\varnothing} 2110=3221,1100 \dot{\varnothing} 2200=3311,1100 \dot{8} 2210=3321,1100 \dot{\varnothing} 3000=4110$, $1100 \dot{\varnothing} 3110=4221,1100 \dot{\wp} 3211=4322,1100 \dot{\wp} 3220=4331,1100 \dot{\wp} 3221=4332,1100 \dot{\wp} 3311=4422$, $1100 \dot{\phi} 3321=4432,1100 \dot{\phi} 4330=4441,1100 \dot{\varphi} 4331=4442,1100 \dot{\phi} 4332=4443,1110 \dot{\varphi} 1110=2221$, $1110 \dot{\varnothing} 2000=3111,1110 \dot{\wp} 2111=3222,1110 \dot{\wp} 2220=3331,1110 \dot{\wp} 3111=4222,1110 \dot{\wp} 3222=4333$, $1110 \dot{\wp} 3331=4442,2000 \dot{\wp} 2100=4210,2000 \dot{\wp} 2200=4220,2000 \dot{\wp} 3100=4310,2000 \dot{\wp} 3110=4311$, $2000 \dot{\varnothing} 3200=4320,2000 \dot{\wp} 3310=4331,2000 \dot{\wp} 3331=4333,2000 \dot{8} 4210=4421,2000 \dot{\wp} 4220=4422$, $2000 \dot{\varnothing} 4310=4431,2000 \dot{\wp} 4320=4432,2000 \dot{\varphi} 4331=4433,2000 \dot{0} 4431=4443,2100 \dot{\wp} 3321=4433$, $2100 \dot{\wp} 4320=4442,2110 \dot{\wp} 3221=4433,2111 \dot{\wp} 2221=4333,2200 \dot{\wp} 3110=4331,2200 \dot{\wp} 3311=4433$, $2200 \dot{\wp} 4220=4442,2210 \dot{\wp} 3211=4433,2220 \dot{\varphi} 3111=4333,3000 \dot{\wp} 3300=4330,3000 \dot{\wp} 4110=4411$, $3000 \dot{\wp} 4330=4433,3100 \dot{\wp} 4310=4442,3110 \dot{\wp} 3110=4422,3110 \dot{\wp} 3220=4433,3110 \dot{\wp} 3310=4442$, $3200 \dot{\varnothing} 4210=4442$ and $3300 \dot{\varnothing} 4110=4441$.

Next, applying $\tilde{s}$ to $S_{4}^{3}$ within $\mathcal{W}_{4}^{N_{3}}$, we obtain the following system of linear equations:

$2 \tilde{s}_{1000}=\tilde{s}_{2100}, \tilde{s}_{1000}+\tilde{s}_{1110}=\tilde{s}_{2111}, \tilde{s}_{1000}+\tilde{s}_{2100}=\tilde{s}_{3210}, \tilde{s}_{1000}+\tilde{s}_{2110}=\tilde{s}_{3211}, \tilde{s}_{1000}+\tilde{s}_{2210}=\tilde{s}_{3221}, \tilde{s}_{1000}+\tilde{s}_{2221}=\tilde{s}_{3222}$, $\tilde{s}_{1000}+\tilde{s}_{3000}=\tilde{s}_{4100}, \tilde{s}_{1000}+\tilde{s}_{3100}=\tilde{s}_{4210}, \tilde{s}_{1000}+\tilde{s}_{3110}=\tilde{s}_{4211}, \tilde{s}_{1000}+\tilde{s}_{3200}=\tilde{s}_{4310}, \tilde{s}_{1000}+\tilde{s}_{3210}=\tilde{s}_{4321}$, $\tilde{s}_{1000}+\tilde{s}_{3321}=\tilde{s}_{4322}, \tilde{s}_{1000}+\tilde{s}_{3320}=\tilde{s}_{4331}, \tilde{s}_{1000}+\tilde{s}_{3321}=\tilde{s}_{4332}, \tilde{s}_{1000}+\tilde{s}_{3332}=\tilde{s}_{4333}, \tilde{s}_{1000}+\tilde{s}_{4300}=\tilde{s}_{4410}$, $\tilde{s}_{1000}+\tilde{s}_{4310}=\tilde{s}_{4421}, \tilde{s}_{1000}+\tilde{s}_{4320}=\tilde{s}_{4431}, \tilde{s}_{1000}+\tilde{s}_{4321}=\tilde{s}_{4432}, \tilde{s}_{1000}+\tilde{s}_{4430}=\tilde{s}_{4441}, \tilde{s}_{1000}+\tilde{s}_{4432}=\tilde{s}_{4443}$, $\tilde{s}_{1000}+\tilde{s}_{4443}=\tilde{s}_{4444}, 2 \tilde{s}_{1100}=\tilde{s}_{2211}, \tilde{s}_{1100}+\tilde{s}_{2211}=\tilde{s}_{3322}, \tilde{s}_{1100}+\tilde{s}_{3100}=\tilde{s}_{4211}, \tilde{s}_{1100}+\tilde{s}_{3200}=\tilde{s}_{4311}, \tilde{s}_{1100}+\tilde{s}_{3300}=\tilde{s}_{4411}$, $\tilde{s}_{1100}+\tilde{s}_{3310}=\tilde{s}_{4421}, \tilde{s}_{1100}+\tilde{s}_{3320}=\tilde{s}_{4431}, \tilde{s}_{1100}+\tilde{s}_{3322}=\tilde{s}_{4433}, \tilde{s}_{1100}+\tilde{s}_{4433}=\tilde{s}_{4444}, \tilde{s}_{1110}+\tilde{s}_{2221}=\tilde{s}_{3332}$, $\tilde{s}_{1110}+\tilde{s}_{3000}=\tilde{s}_{4111}, \tilde{s}_{1110}+\tilde{s}_{3330}=\tilde{s}_{4441}, \tilde{s}_{1110}+\tilde{s}_{3332}=\tilde{s}_{4443}, \tilde{s}_{1110}+\tilde{s}_{4333}=\tilde{s}_{4444}, 2 \tilde{s}_{1111}=\tilde{s}_{2222}, \tilde{s}_{1111}+\tilde{s}_{2222}=\tilde{s}_{3333}$, $\tilde{s}_{1111}+\tilde{s}_{3333}=\tilde{s}_{4444}, 2 \tilde{s}_{2000}+\tilde{s}_{4200}, \tilde{s}_{2000}+\tilde{s}_{2110}=\tilde{s}_{4211}, \tilde{s}_{2000}+\tilde{s}_{2210}=\tilde{s}_{4221}, \tilde{s}_{2000}+\tilde{s}_{2220}=\tilde{s}_{4222}, \tilde{s}_{2000}+\tilde{s}_{3220}=\tilde{s}_{4322}$, $\tilde{s}_{2000}+\tilde{s}_{3320}=\tilde{s}_{4332}, \tilde{s}_{2000}+\tilde{s}_{4200}=\tilde{s}_{4420}, \tilde{s}_{2000}+\tilde{s}_{4420}=\tilde{s}_{4442}, \tilde{s}_{2000}+\tilde{s}_{4442}=\tilde{s}_{4444}, 2 \tilde{s}_{2100}=\tilde{s}_{4321}, \tilde{s}_{2100}+\tilde{s}_{2210}=\tilde{s}_{4322}$, $\tilde{s}_{2100}+\tilde{s}_{3200}=\tilde{s}_{4421}, \tilde{s}_{2100}+\tilde{s}_{3210}=\tilde{s}_{4432}, \tilde{s}_{2100}+\tilde{s}_{4321}=\tilde{s}_{4443}, \tilde{s}_{2100}+\tilde{s}_{4432}=\tilde{s}_{4444}, 2 \tilde{s}_{2110}=\tilde{s}_{4322}, \tilde{s}_{2110}+\tilde{s}_{2210}=\tilde{s}_{4332}$, $\tilde{s}_{2110}+\tilde{s}_{3320}=\tilde{s}_{4442}, \tilde{s}_{2110}+\tilde{s}_{3321}=\tilde{s}_{4443}, \tilde{s}_{2110}+\tilde{s}_{4332}=\tilde{s}_{4444}, \tilde{s}_{2111}+\tilde{s}_{3332}=\tilde{s}_{4444}, 2 \tilde{s}_{2200}=\tilde{s}_{4422}, \tilde{s}_{2200}+\tilde{s}_{4422}=\tilde{s}_{4444}$, $2 \tilde{s}_{2210}=\tilde{s}_{4432}, \tilde{s}_{2210}+\tilde{s}_{3220}=\tilde{s}_{4442}, \tilde{s}_{2210}+\tilde{s}_{3221}=\tilde{s}_{4443}, 2 \tilde{s}_{2211}=\tilde{s}_{4433}, \tilde{s}_{2211}+\tilde{s}_{3322}=\tilde{s}_{4444}, 2 \tilde{s}_{2220}=\tilde{s}_{4442}$, $\tilde{s}_{2220}+\tilde{s}_{3111}=\tilde{s}_{4333}, \tilde{s}_{2220}+\tilde{s}_{4222}=\tilde{s}_{4444}, 2 \tilde{s}_{2221}=\tilde{s}_{4443}, \tilde{s}_{2221}+\tilde{s}_{3222}=\tilde{s}_{4444}, 2 \tilde{s}_{2222}=\tilde{s}_{4444}, 2 \tilde{s}_{3000}=\tilde{s}_{4300}$, $\tilde{s}_{3000}+\tilde{s}_{3330}=\tilde{s}_{4333}, \tilde{s}_{3000}+\tilde{s}_{4100}=\tilde{s}_{4410}, \tilde{s}_{3000}+\tilde{s}_{4300}=\tilde{s}_{4430}, \tilde{s}_{3000}+\tilde{s}_{4410}=\tilde{s}_{4441}, \tilde{s}_{3000}+\tilde{s}_{4430}=\tilde{s}_{4443}$, $\tilde{s}_{3000}+\tilde{s}_{4441}=\tilde{s}_{4444}, 2 \tilde{s}_{3100}=\tilde{s}_{4421}, \tilde{s}_{3100}+\tilde{s}_{3200}=\tilde{s}_{4431}, \tilde{s}_{3100}+\tilde{s}_{3320}=\tilde{s}_{4433}, \tilde{s}_{3100}+\tilde{s}_{4320}=\tilde{s}_{4443}, \tilde{s}_{3100}+\tilde{s}_{4431}=\tilde{s}_{4444}$, $\tilde{s}_{3110}+\tilde{s}_{3320}=\tilde{s}_{4443}, \tilde{s}_{3110}+\tilde{s}_{4331}=\tilde{s}_{4444}, \tilde{s}_{3111}+\tilde{s}_{3331}=\tilde{s}_{4444}, 2 \tilde{s}_{3200}=\tilde{s}_{4432}, \tilde{s}_{3200}+\tilde{s}_{3310}=\tilde{s}_{4433}, \tilde{s}_{3200}+\tilde{s}_{4310}=\tilde{s}_{4443}$, $\tilde{s}_{3200}+\tilde{s}_{4421}=\tilde{s}_{4444}, 2 \tilde{s}_{3210}=\tilde{s}_{4443}, \tilde{s}_{3210}+\tilde{s}_{4321}=\tilde{s}_{4444}, \tilde{s}_{3211}+\tilde{s}_{3321}=\tilde{s}_{4444}, \tilde{s}_{3220}+\tilde{s}_{4221}=\tilde{s}_{4444}, 2 \tilde{s}_{3221}=\tilde{s}_{4444}$, 
$2 \tilde{s}_{3300}=\tilde{s}_{4433}, \tilde{s}_{3300}+\tilde{s}_{4411}=\tilde{s}_{4444}, \tilde{s}_{3310}+\tilde{s}_{4311}=\tilde{s}_{4444}, 2 \tilde{s}_{3311}=\tilde{s}_{4444}, \tilde{s}_{3320}+\tilde{s}_{4211}=\tilde{s}_{4444}, \tilde{s}_{3330}+\tilde{s}_{4111}=\tilde{s}_{4444}$, $2 \tilde{s}_{4000}=\tilde{s}_{4400}, \tilde{s}_{4000}+\tilde{s}_{4400}=\tilde{s}_{4440}, \tilde{s}_{4000}+\tilde{s}_{4440}=\tilde{s}_{4444}, \tilde{s}_{4100}+\tilde{s}_{4300}=\tilde{s}_{4441}, \tilde{s}_{4100}+\tilde{s}_{4430}=\tilde{s}_{4444}, \tilde{s}_{4110}+\tilde{s}_{4330}=\tilde{s}_{4444}$, $2 \tilde{s}_{4200}=\tilde{s}_{4442}, \tilde{s}_{4200}+\tilde{s}_{4420}=\tilde{s}_{4444}, \tilde{s}_{4210}+\tilde{s}_{4320}=\tilde{s}_{4444}, 2 \tilde{s}_{4220}=\tilde{s}_{4444}, 2 \tilde{s}_{4300}=\tilde{s}_{4443}, \tilde{s}_{4300}+\tilde{s}_{4410}=\tilde{s}_{4444}$, $2 \tilde{s}_{4310}=\tilde{s}_{4444}$ and $2 \tilde{s}_{4400}=\tilde{s}_{4444}$.

Moreover, we have the extension property: $\tilde{s}_{0000}=s_{0}=0, \tilde{s}_{1111}=s_{1}=1 / 4, \tilde{s}_{2222}=s_{2}=1 / 2, \tilde{s}_{3333}=s_{3}=3 / 4$ and $\tilde{s}_{4444}=1$. It appears that the system with these initial conditions is not complete with $\tilde{s}_{4330}$ as a parameter $p$ such that $4 / 7 \leq p \leq 87 / 140$. To supplement the system, we are looking for a consistent equation by applying $\tilde{s}$ to suitable deflected partial products. We discover that

(1) the system of linear equations together with an equation $\tilde{s}_{1100}+\tilde{s}_{3000}=\tilde{s}_{4110}$ and

(2) the system together with an equation $\tilde{s}_{3000}+\tilde{s}_{3300}=\tilde{s}_{4330}$ form two complete systems of linear equations yielding the required solutions.

Finally, in view of these solutions, applying $\tilde{s}$ to all deflected partial products, we conclude a verification of the assertions of the theorem.

Note that the 3-extension $\mathcal{W}_{4}^{N_{3}}$ of the Weber $M V$-chain $\mathcal{W}_{4}$ contains the following ten Weber $M V$-chains:

$0000<1000<1000^{2 \dot{\phi}}(=2100)<1000^{3 \dot{\phi}}(=3210)<1000^{4 \dot{\varphi}}(=4321)<1000^{5 \dot{\phi}}(=4432)<1000^{6 \dot{\rho}}(=4443)<$ $1000^{7 \dot{\varphi}}(=4444), 0000<1100<1100^{2 \dot{\varphi}}(=2211)<1100^{3 \dot{\varphi}}(=3322)<1100^{4 \dot{\varphi}}(=4433)<1100^{5 \dot{\varphi}}(=4444)$, $0000<1111<1111^{2 \dot{\phi}}(=2222)<1111^{3 \dot{\phi}}(=3333)<1111^{4 \dot{\varphi}}(=4444), 0000<2000<2000^{2 \dot{\phi}}(=4200)<$ $2000^{3 \dot{\dot{\varphi}}}(=4420)<2000^{4 \dot{\varphi}}(=4442)<2000^{5 \dot{\varphi}}(=4444), 0000<2200<2200^{2 \dot{\varphi}}(=4422)<2200^{3 \dot{\phi}}(=4444)$, $0000<3221<3221^{2 \dot{\varphi}}(=4444), 0000<3311<3311^{2 \dot{\varphi}}(=4444), 0000<4000<4000^{2 \dot{\varphi}}(=4400)<4000^{3 \dot{\varphi}}(=$ $4440)<4000^{4 \dot{\varphi}}(=4444), 0000<4220<4220^{2 \dot{\phi}}(=4444)$ and $0000<4310<4310^{2 \dot{\phi}}(=4444)$.

\section{Proof of Theorem 16}

We have that $\mathcal{W}_{2}^{N_{4}}$ consists of twenty one elements: $00000<10000<11000<11100<11110<11111$, $20000<21000<21100<21110<21111,22000<22100<22110<22111,22200<22210<22211$, $22220<22221<22222$. An ordinary calculation shows that the set $E_{2}^{4}$ of all existing partial binary products within $\mathcal{W}_{2}^{N_{4}}$ is as follows:

$10000 \dot{\wp} 10000=21000,10000 \dot{\wp} 11000=21100,10000 \dot{\wp} 11100=21110,10000 \dot{\wp} 11110=21111,10000 \dot{\wp} 21000=$ $22100,10000 \dot{\wp} 21100=22110,10000 \wp 21110=22111,10000 \dot{\wp} 22100=22210,10000 \dot{8} 22110=22211$, $10000 \dot{\varnothing} 22210=22221,10000 \dot{\wp} 22221=22222,11000 \dot{\wp} 11000=22110,11000 \dot{\wp} 11100=22111,11000 \dot{\wp} 21100=$ $22211,11000 \dot{\wp} 22110=22221,11000 \dot{\wp} 22211=22222,11100 \dot{\wp} 11100=22211,11100 \dot{\phi} 21110=22221$, $11100 \dot{\varnothing} 22111=22222,11110 \dot{\wp} 11110=22221,11110 \dot{\wp} 21111=22222,11111 \dot{\wp} 11111=22222,20000 \dot{\wp} 20000=$ $22000,20000 \dot{\wp} 22000=22200,20000 \dot{\wp} 22200=22220,20000 \dot{\wp} 22220=22222,21000 \dot{\wp} 21000=22210$, $21000 \dot{\wp} 22100=22221,21000 \dot{\wp} 22210=22222,21100 \dot{\wp} 21100=22221,21100 \dot{\wp} 22110=22222,21110 \dot{\wp} 21110=$ $22222,22000 \dot{\wp} 22000=22220,22000 \dot{\wp} 22200=22222$ and $22100 \wp 22100=22222$.

Next, verifying each of the partial triple products in turn, we establish the following six exclusive situations:

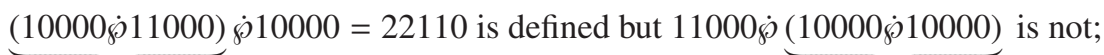

$$
=21100 \quad \underbrace{10000 \varphi 010000}_{=21000}
$$
$\underbrace{(10000 \dot{\wp} 11000)}_{=21100} \dot{\wp} 21100=11000 \dot{\wp} \underbrace{(10000 \dot{\phi} 21100)}_{=22110}=22221$ are defined but $10000 \dot{\wp} \underbrace{(11000 \dot{\wp} 21100)}_{=22211}$ is not;

$\underbrace{(10000 \dot{\wp} 11100)}_{=21110} \dot{\wp} 10000=22111$ is defined but $11100 \dot{\wp} \underbrace{(10000 \dot{\wp} 10000)}_{=21000}$ is not;

$\underbrace{\wp}_{(10000 \dot{\wp} 11100)} \dot{\wp} 11100=22221$ is defined but $10000 \dot{\wp}(11100 \dot{\wp} 11100)$ is not;

$$
=21110 \quad \underbrace{(11100 \dot{\rho} 11100)}_{=22211} \text { is not; }
$$

$\underbrace{(10000 \dot{\wp} 21100)}_{=22110} \dot{\wp} 10000=22211$ is defined but $21100 \dot{\wp} \underbrace{(10000 \dot{\wp} 10000)}_{=21000}$ is not;

$\underbrace{(10000 \dot{\wp} 21100)} \dot{\wp} 11000=21100 \dot{\wp} \underbrace{(10000 \dot{\wp} 11000)}=22221$ are defined but $10000 \dot{\wp}(11000 \dot{\wp} 21100)$ is not.

$$
=22110=21100 \quad 22211
$$

Now gathering all partial binary products from existing partial triple products, we form the set $D_{2}^{4}$ within $W_{2}^{\mathcal{N}_{4}}$; it consists of the following deflected partial products: 
$10000 \dot{\wp} 11000=21100,10000 \dot{\wp} 11100=21110,10000 \dot{\wp} 21100=22110,10000 \dot{\wp} 21110=22111,10000 \dot{\wp} 22110=$

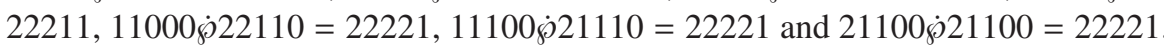

Now applying $\tilde{s}$ to all steady partial products within $\mathcal{W}_{2}^{\mathcal{N}_{4}}$ we obtain the following system of linear equations:

$2 \tilde{s}_{10000}=\tilde{s}_{21000}, \tilde{s}_{10000}+\tilde{s}_{11110}=\tilde{s}_{21111}, \tilde{s}_{10000}+\tilde{s}_{21000}=\tilde{s}_{22100}, \tilde{s}_{10000}+\tilde{s}_{22100}=\tilde{s}_{22210}, \tilde{s}_{10000}+\tilde{s}_{22210}=\tilde{s}_{22221}$, $\tilde{s}_{10000}+\tilde{s}_{22221}=\tilde{s}_{22222}, 2 \tilde{s}_{11000}=\tilde{s}_{22110}, \tilde{s}_{11000}+\tilde{s}_{11100}=\tilde{s}_{22111}, \tilde{s}_{11000}+\tilde{s}_{21100}=\tilde{s}_{22211}, \tilde{s}_{11000}+\tilde{s}_{22211}=\tilde{s}_{22222}$, $2 \tilde{s}_{11100}=\tilde{s}_{22211}, \tilde{s}_{11100}+\tilde{s}_{22111}=\tilde{s}_{22222}, 2 \tilde{s}_{11110}=\tilde{s}_{22221}, \tilde{s}_{11110}+\tilde{s}_{21111}=\tilde{s}_{22222}, 2 \tilde{s}_{11111}=\tilde{s}_{22222}, 2 \tilde{s}_{20000}=\tilde{s}_{22000}$, $\tilde{s}_{20000}+\tilde{s}_{22000}=\tilde{s}_{22200}, \tilde{s}_{20000}+\tilde{s}_{22200}=\tilde{s}_{22220}, \tilde{s}_{20000}+\tilde{s}_{22220}=\tilde{s}_{22222}, 2 \tilde{s}_{21000}=\tilde{s}_{22210}, \tilde{s}_{21000}+\tilde{s}_{22100}=\tilde{s}_{22221}$, $\tilde{s}_{21000}+\tilde{s}_{22210}=\tilde{s}_{22222}, \tilde{s}_{21100}+\tilde{s}_{22110}=\tilde{s}_{22222}, 2 \tilde{s}_{21110}=\tilde{s}_{22222}, 2 \tilde{s}_{22000}=\tilde{s}_{22220}, \tilde{s}_{22000}+\tilde{s}_{22200}=\tilde{s}_{22222}$ and $2 \tilde{s}_{22100}=\tilde{s}_{22222}$.

Moreover, we have that $\tilde{s}_{00000}=s_{0}=0, \tilde{s}_{11111}=s_{1}=1 / 2$ and $\tilde{s}_{22222}=s_{2}=1$. It appears that this system of linear equations is not complete with $\tilde{s}_{11000}$ as a parameter $p$ such that $1 / 4 \leq p \leq 1 / 3$ (by the isotonicity of $\tilde{s}$ ). To supplement the system, we are looking for consistent equations by applying $\tilde{s}$ to suitable deflected partial binary products. We discover that

(1) the system of linear equations together with an equation $\tilde{s}_{10000}+\tilde{s}_{11000}=\tilde{s}_{21100}$,

(2) the system together with an equation $2 \tilde{s}_{21100}=\tilde{s}_{22221}$ and

(3) the system together with an equation $\tilde{s}_{10000}+\tilde{s}_{11100}=\tilde{s}_{21110}$

form three complete system of linear equations yielding the required solutions.

Finally, in view of these solutions, applying $\tilde{s}$ to all deflected partial binary products, we conclude a verification of the assertions of the theorem.

Note that the 4-extension $\mathcal{W}_{2}^{\mathrm{N}_{4}}$ of the Weber $M V$-chain $\mathcal{W}_{2}$ contains the following six Weber $M V$-chains:

$00000<10000<10000^{2 \dot{\varphi}}(=21000)<10000^{3 \dot{\varphi}}(=22100)<10000^{4 \dot{\varphi}}(=22210)<10000^{5 \dot{\varphi}}(=22221)<$ $10000^{6 \dot{\varphi}}(=22222), 00000<11111<11111^{2 \dot{\varphi}}(=22222), 00000<20000<20000^{2 \dot{\varphi}}(=22000)<20000^{3 \dot{\varphi}}(=$ $22200)<20000^{4 \dot{\varphi}}(=22200)<20000^{5 \dot{\varphi}}(=22222), 00000<21000<21000^{2 \dot{\varphi}}(=22210)<21000^{3 \dot{\varphi}}(=22222)$, $00000<21110<21110^{2 \dot{\varphi}}(=22222)$ and $00000<22100<22100^{2 \dot{\phi}}(=22222)$.

\section{Proof of Theorem 17}

We have that $W_{3}^{\mathcal{N}_{4}}$ consists of fifty six elements: $00000<10000<11000<11100<11110<11111,20000<$ $21000<21100<21110<21111,22000<22100<22110<22111,22200<22210<22211,22220<22221<$ $22222,30000<31000<31100<31110<31111,32000<32100<32110<32111,32200<32210<32211$, $32220<32221<32222,33000<33100<33110<33111,33200<33210<33211,33220<33221<33222$, $33300<33310<33311,33320<33321<33322,33330<33331<33332<33333$. A routine calculation shows that the set $E_{3}^{4}$ of all existing partial binary products within $\mathcal{W}_{3}^{\mathcal{N}_{4}}$ is as follows:

$10000 \dot{\wp} 10000=21000,10000 \dot{\wp} 11000=21100,10000 \dot{\wp} 11100=21110,10000 \dot{\wp} 11110=21111,10000 \dot{\wp} 20000=$ $31000,10000 \dot{\wp} 21000=32100,10000 \dot{\wp} 21100=32110,10000 \dot{\phi} 21110=32111,10000 \dot{\wp} 22100=32210$, $10000 \dot{\wp} 22110=32211,10000 \dot{\varnothing} 22210=32221,10000 \dot{\wp} 22221=32222,10000 \dot{\wp} 32000=33100,10000 \dot{\wp} 32100=$ $33210,10000 \dot{\wp} 32110=33211,10000 \dot{\varnothing} 32210=33221,10000 \dot{\oplus} 32221=33222,10000 \dot{\wp} 33200=33310$,

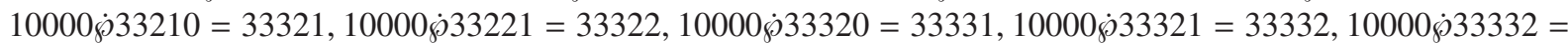
$33333,11000 \dot{\wp} 11000=22110,11000 \dot{\wp} 11100=22111,11000 \dot{8} 20000=31100,11000 \dot{\wp} 21000=32110$,

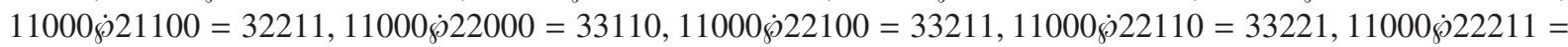
$33222,11000 \dot{\wp} 32200=33311,11000 \dot{\wp} 32210=33321,11000 \dot{\wp} 32211=33322,11000 \dot{\wp} 33220=33331$, $11000 \dot{\wp} 33221=33332,11000 \dot{\varnothing} 33322=33333,11100 \dot{\wp} 11100=22211,11100 \dot{\wp} 20000=31110,11100 \dot{\varnothing} 21000=$ $32111,11100 \dot{\wp} 21110=32221,11100 \dot{\varnothing} 22000=33111,11100 \dot{\varnothing} 22111=33222,11100 \dot{\varnothing} 22200=33311$, $11100 \dot{\wp} 22210=33321,11100 \dot{\varnothing} 22211=33322,11100 \dot{\wp} 32220=33331,11100 \dot{\wp} 32221=33332,11100 \dot{0} 33222=$ $33333,11110 \dot{\wp} 11110=22221,11110 \dot{\varnothing} 20000=31111,11110 \dot{\varnothing} 21111=32222,11110 \dot{\wp} 22220=33331$, $11110 \dot{\wp} 22221=33332,11110 \dot{\wp} 32222=33333,11111 \dot{\wp} 11111=22222,11111 \dot{\wp} 22222=33333,20000 \dot{\varphi} 20000=$ $32000,20000 \dot{\wp} 22000=32200,20000 \dot{\wp} 22200=32220,20000 \dot{\varnothing} 22220=32222,20000 \dot{\wp} 31000=33100$, $20000 \dot{\wp} 31100=33110,20000 \dot{\wp} 31110=33111,20000 \dot{\wp} 32000=33200,20000 \dot{\wp} 32200=33220,20000 \dot{0} 32220=$ $33222,20000 \dot{\wp} 33100=33310,20000 \dot{\wp} 33110=33311,20000 \dot{8} 33200=33320,20000 \dot{\wp} 33220=33322$, $20000 \dot{\wp} 33310=33331,20000 \dot{\varnothing} 33320=33332,20000 \dot{\wp} 33331=33333,21000 \dot{\wp} 21000=33210,21000 \dot{\varphi} 21100=$ $33211,21000 \dot{\wp} 22100=33221,21000 \dot{\wp} 22210=33222,21000 \dot{8} 32100=33321,21000 \dot{\wp} 32210=33322$, $21000 \dot{\wp} 33210=33332,21000 \dot{\varnothing} 33321=33333,21100 \dot{\wp} 21100=33221,21100 \dot{\wp} 22100=33321,21100 \dot{\varnothing} 22110=$ 
$33322,21100 \dot{\varnothing} 32210=33332,21100 \dot{\wp} 33221=33333,21110 \dot{\varnothing} 21110=33222,21110 \dot{\varphi} 22210=33332$, $21110 \dot{\wp} 32221=33333,21111 \dot{\wp} 22221=33333,22000 \dot{\wp} 22000=33220,22000 \dot{\wp} 22200=33222,22000 \dot{\wp} 31100=$ $33311,22000 \dot{\wp} 32200=33322,22000 \dot{\varnothing} 33110=33331,22000 \dot{\varphi} 33311=33333,22100 \dot{\varphi} 22100=33322$, $22100 \dot{\wp} 32110=33332,22100 \dot{\wp} 33211=33333,22110 \dot{\wp} 22110=33332,22110 \dot{\wp} 32211=33333,22111 \dot{\varnothing} 22211=$

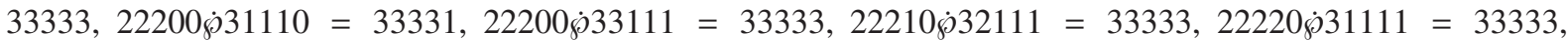
$30000 \dot{\wp} 30000=33000,30000 \dot{\wp} 33000=33300,30000 \dot{\wp} 33300=33330,30000 \dot{\wp} 33330=33333,31000 \dot{\varnothing} 32000=$ $33310,31000 \dot{\varnothing} 33200=33331,31000 \dot{\wp} 33320=33333,31100 \dot{\wp} 32200=33331,31100 \dot{\wp} 33220=33333$,

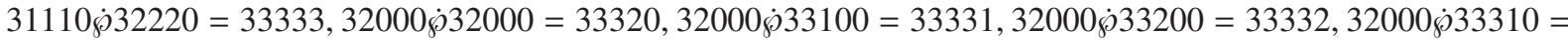
$33333,32100 \dot{\wp} 32100=33332,32100 \dot{\wp} 33210=33333,32110 \dot{\wp} 32210=33333,32200 \dot{\wp} 33110=33333$,

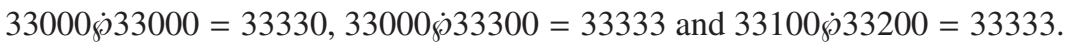

Next, verifying each of the partial triple products in turn, we discover the following twenty six "non-associative" situations:

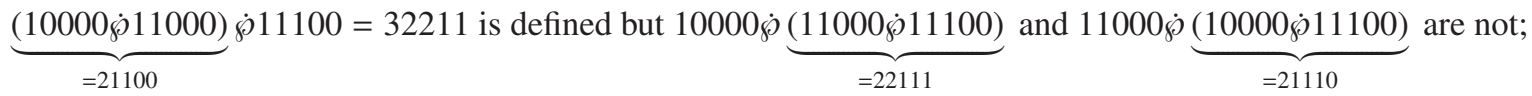

$\underbrace{(10000 \dot{\wp} 11000)}_{=21100} \dot{\phi} 21000=10000 \dot{\wp} \underbrace{(11000 \dot{\wp} 21000)}_{=32110}=33211$ are defined but $11000 \dot{\wp} \underbrace{(10000 \dot{\wp} 21000)}_{=32100}$ is not;

$\underbrace{\wp}_{(10000 \dot{\wp} 11000)} 21100=33221$ is defined but $10000 \dot{\wp}(11000 \dot{\wp} 21100)$ and $11000 \dot{\wp}(10000 \dot{\wp} 21100)$ are not; $=21100$ $=32211$ $=32110$

$\underbrace{(10000 \dot{\wp} 11100)}_{=21110} \dot{\wp} 11100=32221$ is defined but $10000 \dot{\wp} \underbrace{(11100 \dot{\wp} 11100)}_{=22211}$ is not;

$\underbrace{(10000 \dot{\wp} 11100)} \dot{\wp} 21110=10000 \dot{\wp}(\underbrace{(11100 \dot{8} 21110)}=33222$ are defined but $11100 \dot{\wp}(10000 \dot{\wp} 21110)$ is not;

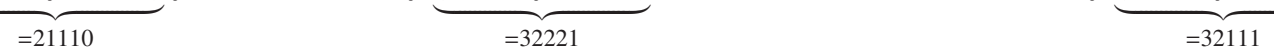
$\underbrace{(10000 \dot{\wp} 22100)}_{=32210} \dot{\wp} 11000=22100 \dot{\phi} \underbrace{(10000 \dot{\wp} 11000)}_{=21100}=33321$ are defined but $10000 \dot{\wp} \underbrace{(11000 \dot{\wp} 22100)}_{=33211}$ is not;

$\underbrace{(10000 \dot{\wp} 22100)}_{=32210} \dot{\wp} 21000=10000 \dot{\wp} \underbrace{(21000 \dot{\phi} 22100)}_{=33221}=33322$ are defined but $22100 \dot{\phi} \underbrace{(10000 \dot{\phi} 21000)}_{=32100}$ is not;

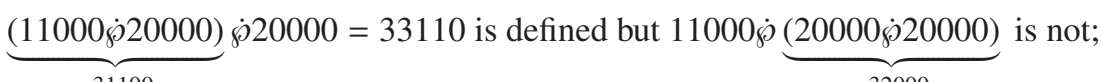

$$
=31100 \quad \underbrace{20000 \dot{20000)}}_{=32000}
$$

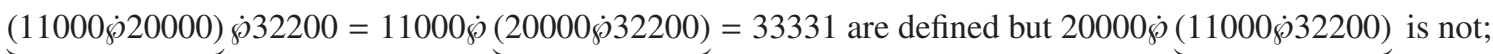
$=31100 \quad \underbrace{20000 \dot{3}}_{=33220}$ $\underbrace{(11000 \dot{\wp} 21000)}_{=32110} \dot{\wp} 10000=21000 \dot{\wp} \underbrace{(10000 \dot{\wp} 11000)}_{=21100}=33211$ are defined but $11000 \dot{\wp} \underbrace{(10000 \dot{\wp} 21000)}_{=32100}$ is not; $\underbrace{(11000 \dot{\wp} 21000)}_{=32110} \dot{\wp} 22100=11000 \dot{\wp} \underbrace{(21000 \dot{\phi} 22100)}_{=33221}=33332$ are defined but $21000 \dot{\phi} \underbrace{(11000 \dot{\varphi} 22100)}_{=33211}$ is not; $\underbrace{(11100 \dot{\wp} 20000)}_{=31110} \dot{\wp} 20000=33111$ is defined but $11100 \dot{\phi} \underbrace{(20000 \dot{\gamma} 20000)}_{=32000}$ is not;

$\underbrace{(11100 \dot{\wp} 21110)} \dot{\wp} 10000=21110 \dot{\wp} \underbrace{(10000 \dot{\wp} 11100)}=33222$ are defined but $11100 \dot{\wp}(10000 \dot{\wp} 21110)$ is not; $=32221 \quad \underbrace{10000 \dot{0} 11100)}_{=21110} 33222$ are defin

$\underbrace{(11100 \dot{\wp} 21110)}_{-32221} \dot{\wp} 11100=33332$ is defined but $21110 \dot{\rho}(\underbrace{(11100 \dot{\wp} 11100)}$ is not; $=32221 \quad \underbrace{11100}_{=22211}$

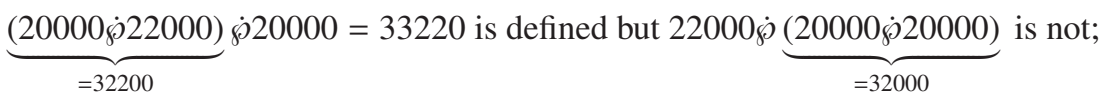

$(20000 \dot{\wp} 22000) \dot{\wp} 31100=22000 \dot{\wp}(20000 \dot{\varnothing} 31100)=33331$ are defined but $20000 \dot{\wp}(22000 \dot{\varnothing} 31100)$ is not;

$$
=32200=33110 \quad \underbrace{20000}_{=33311}
$$

$\underbrace{(20000 \dot{\wp} 22200)} \dot{\wp} 11100=22200 \dot{\wp}(11100 \dot{\varnothing} 20000)=33331$ are defined but $20000 \dot{\wp}(11100 \dot{\wp} 22200)$ is not; $=32220$ 
$\underbrace{(20000 \dot{\wp} 22200)}_{=32220} \dot{\wp} 20000=33222$ is defined but $22200 \dot{\phi} \underbrace{(20000 \dot{\gamma} 20000)}_{=32000}$ is not;

$\underbrace{(20000 \dot{\wp} 31100)}_{=33110} \dot{\wp} 20000=33311$ is defined but $31100 \dot{\wp} \underbrace{(20000 \dot{\wp} 20000)}_{=32000}$ is not;

$\underbrace{(20000 \dot{\wp} 31100)}_{=33110} \dot{\wp} 22000=31100 \dot{\wp} \underbrace{(20000 \dot{\wp} 22000)}_{=32200}=33331$ are defined but $20000 \dot{\wp} \underbrace{(22000 \dot{\wp} 31100)}_{=33311}$ is not; $\underbrace{(20000 \dot{\wp} 32200)} \dot{\wp} 11000=32200 \dot{\wp}(11000 \dot{\varnothing} 20000)=33331$ are defined but $20000 \dot{\wp}(11000 \dot{\wp} 32200)$ is not; $=33220=31100 \quad \underbrace{}_{=33311}$ $\underbrace{(20000 \dot{\wp} 32200)}_{=33220} \dot{\wp} 20000=33322$ is defined but $32200 \dot{\phi} \underbrace{(20000 \dot{\varphi} 20000)}_{=32000}$ is not; $\underbrace{(21000 \dot{\wp} 22100)}_{=33221} \dot{\wp} 10000=21000 \dot{\wp} \underbrace{(10000 \dot{\wp} 22100)}_{=32210}=33322$ are defined but $22100 \dot{\wp} \underbrace{(10000 \dot{\wp} 21000)}_{=32100}$ is not; $\underbrace{(21000 \dot{\wp} 22100)}_{=33221} \dot{\wp} 11000=22100 \dot{\wp} \underbrace{(11000 \dot{\phi} 21000)}_{=32110}=33332$ are defined but $21000 \dot{\phi} \underbrace{(11000 \dot{\varphi} 22100)}_{=33211}$ is not; $\underbrace{(21100 \dot{\wp} 21100)}_{=33221} \dot{\wp} 10000=33322$ is defined but $21100 \dot{\wp} \underbrace{(10000 \dot{\wp} 21100)}_{=32110}$ is not; $\underbrace{(21100 \dot{\wp} 21100)}_{=33221} \dot{\wp} 11000=33332$ is defined but $21100 \dot{\varnothing}(\underbrace{(11000 \dot{\gamma} 21100)}_{=32211}$ is not.

Further, gathering all partial binary products from existing partial triple products, we form the set $D_{3}^{4}$ within $\mathcal{W}_{3}^{\mathcal{N}_{4}}$; it consists of the following deflected partial products:

$10000 \dot{\wp} 11000=21100,10000 \dot{\wp} 11100=21110,10000 \dot{\wp} 22100=32210,10000 \dot{\wp} 32110=33211,10000 \dot{\wp} 32221=$ $33222,10000 \dot{\wp} 33221=33322,11000 \dot{\varnothing} 20000=31100,11000 \dot{\varnothing} 21000=32110,11000 \dot{\wp} 32210=33321$, $11000 \dot{\wp} 32211=33322,11000 \dot{\wp} 33220=33331,11000 \dot{\wp} 33221=33332,11100 \dot{\wp} 20000=31110,11100 \dot{\varphi} 21110=$ $32221,11100 \dot{\phi} 32220=33331,11100 \dot{\wp} 32221=33332,20000 \dot{\phi} 22000=32200,20000 \dot{\wp} 22200=32220$, $20000 \dot{\wp} 31100=33110,20000 \dot{\wp} 31110=33111,20000 \dot{8} 32200=33220,20000 \dot{\wp} 32220=33222,20000 \dot{\wp} 33110=$ $33311,20000 \dot{\wp} 33220=33322,21000 \dot{\wp} 21100=33211,21000 \dot{8} 22100=33221,21000 \dot{\wp} 32210=33322$, $21100 \dot{\wp} 21100=33221,21100 \dot{\wp} 22100=33321,21110 \dot{\wp} 21110=33222,22000 \dot{\wp} 33110=33331,22100 \dot{0} 32110=$ $33332,22200 \dot{\wp} 31110=33331$ and $31100 \dot{\wp} 32200=33331$.

Now applying $\tilde{s}$ to all steady partial products within $\mathcal{W}_{3}^{\mathcal{N}_{4}}$, we obtain the following system of linear equations:

$2 \tilde{s}_{10000}=\tilde{s}_{21000}, \tilde{s}_{10000}+\tilde{s}_{1110}=\tilde{s}_{21111}, \tilde{s}_{10000}+\tilde{s}_{20000}=\tilde{s}_{31000}, \tilde{s}_{10000}+\tilde{s}_{21000}=\tilde{s}_{32100}, \tilde{s}_{10000}+\tilde{s}_{21100}=\tilde{s}_{32110}$, $\tilde{s}_{10000}+\tilde{s}_{21110}=\tilde{s}_{32111}, \tilde{s}_{10000}+\tilde{s}_{22110}=\tilde{s}_{32211}, \tilde{s}_{10000}+\tilde{s}_{22210}=\tilde{s}_{32221}, \tilde{s}_{10000}+\tilde{s}_{22221}=\tilde{s}_{32222}, \tilde{s}_{10000}+\tilde{s}_{32000}=\tilde{s}_{33100}$, $\tilde{s}_{10000}+\tilde{s}_{32100}=\tilde{s}_{33210}, \tilde{s}_{10000}+\tilde{s}_{32210}=\tilde{s}_{33221}, \tilde{s}_{10000}+\tilde{s}_{33200}=\tilde{s}_{33310}, \tilde{s}_{10000}+\tilde{s}_{33210}=\tilde{s}_{33321}, \tilde{s}_{10000}+\tilde{s}_{33320}=\tilde{s}_{33331}$, $\tilde{s}_{10000}+\tilde{s}_{33321}=\tilde{s}_{33332}, \tilde{s}_{10000}+\tilde{s}_{33332}=\tilde{s}_{33333}, 2 \tilde{s}_{11000}=\tilde{s}_{22110}, \tilde{s}_{11000}+\tilde{s}_{11100}=\tilde{s}_{22111}, \tilde{s}_{11000}+\tilde{s}_{21100}=\tilde{s}_{32211}$, $\tilde{s}_{11000}+\tilde{s}_{22000}=\tilde{s}_{33110}, \tilde{s}_{11000}+\tilde{s}_{22100}=\tilde{s}_{33211}, \tilde{s}_{11000}+\tilde{s}_{22110}=\tilde{s}_{33221}, \tilde{s}_{11000}+\tilde{s}_{22211}=\tilde{s}_{33222}, \tilde{s}_{11000}+\tilde{s}_{32200}=\tilde{s}_{33311}$, $\tilde{s}_{11000}+\tilde{s}_{33322}=\tilde{s}_{33333}, 2 \tilde{s}_{11100}=\tilde{s}_{22211}, \tilde{s}_{11100}+\tilde{s}_{21000}=\tilde{s}_{32111}, \tilde{s}_{11100}+\tilde{s}_{22000}=\tilde{s}_{33111}, \tilde{s}_{11100}+\tilde{s}_{22111}=\tilde{s}_{33222}$, $\tilde{s}_{11100}+\tilde{s}_{22200}=\tilde{s}_{33311}, \tilde{s}_{11100}+\tilde{s}_{22210}=\tilde{s}_{33321}, \tilde{s}_{11100}+\tilde{s}_{22211}=\tilde{s}_{33322}, \tilde{s}_{11100}+\tilde{s}_{33222}=\tilde{s}_{33333}, 2 \tilde{s}_{11110}=\tilde{s}_{22221}$, $\tilde{s}_{11110}+\tilde{s}_{20000}=\tilde{s}_{31111}, \tilde{s}_{11110}+\tilde{s}_{21111}=\tilde{s}_{32222}, \tilde{s}_{11110}+\tilde{s}_{22220}=\tilde{s}_{33331}, \tilde{s}_{11110}+\tilde{s}_{22221}=\tilde{s}_{33332}, \tilde{s}_{11110}+\tilde{s}_{32222}=\tilde{s}_{33333}$, $2 \tilde{s}_{20000}=\tilde{s}_{32000}, \tilde{s}_{20000}+\tilde{s}_{22220}=\tilde{s}_{32222}, \tilde{s}_{20000}+\tilde{s}_{31000}=\tilde{s}_{33100}, \tilde{s}_{20000}+\tilde{s}_{32000}=\tilde{s}_{33200}, \tilde{s}_{20000}+\tilde{s}_{33100}=\tilde{s}_{33310}$, $\tilde{s}_{20000}+\tilde{s}_{33200}=\tilde{s}_{33320}, \tilde{s}_{20000}+\tilde{s}_{33310}=\tilde{s}_{33331}, \tilde{s}_{20000}+\tilde{s}_{33320}=\tilde{s}_{33332}, \tilde{s}_{20000}+\tilde{s}_{33331}=\tilde{s}_{33333}, 2 \tilde{s}_{21000}=\tilde{s}_{33210}$, $\tilde{s}_{21000}+\tilde{s}_{22210}=\tilde{s}_{33222}, \tilde{s}_{21000}+\tilde{s}_{32100}=\tilde{s}_{33321}, \tilde{s}_{21000}+\tilde{s}_{33210}=\tilde{s}_{33332}, \tilde{s}_{21000}+\tilde{s}_{33321}=\tilde{s}_{33333}, \tilde{s}_{21100}+\tilde{s}_{22110}=\tilde{s}_{33322}$, $\tilde{s}_{21100}+\tilde{s}_{32210}=\tilde{s}_{33332}, \tilde{s}_{21100}+\tilde{s}_{33221}=\tilde{s}_{33333}, \tilde{s}_{21110}+\tilde{s}_{22210}=\tilde{s}_{33332}, \tilde{s}_{21110}+\tilde{s}_{32221}=\tilde{s}_{33333}, \tilde{s}_{21111}+\tilde{s}_{22221}=\tilde{s}_{33333}$, $2 \tilde{s}_{22000}=\tilde{s}_{33220}, \tilde{s}_{22000}+\tilde{s}_{22200}=\tilde{s}_{33222}, \tilde{s}_{22000}+\tilde{s}_{31100}=\tilde{s}_{33311}, \tilde{s}_{22000}+\tilde{S}_{32200}=\tilde{s}_{33322}, \tilde{s}_{22000}+\tilde{s}_{33311}=\tilde{s}_{33333}$, $2 \tilde{s}_{22100}=\tilde{s}_{33322}, \tilde{s}_{22100}+\tilde{s}_{33211}=\tilde{s}_{33333}, 2 \tilde{s}_{22110}=\tilde{s}_{33332}, \tilde{s}_{22110}+\tilde{s}_{32211}=\tilde{s}_{33333}, \tilde{s}_{22111}+\tilde{s}_{22211}=\tilde{s}_{33333}$, $\tilde{s}_{22200}+\tilde{s}_{33111}=\tilde{s}_{33333}, \tilde{s}_{22210}+\tilde{s}_{32111}=\tilde{s}_{33333}, \tilde{s}_{22220}+\tilde{s}_{31111}=\tilde{s}_{33333}, 2 \tilde{s}_{30000}=\tilde{s}_{33000}, \tilde{s}_{30000}+\tilde{s}_{33000}=\tilde{s}_{33300}$, $\tilde{s}_{30000}+\tilde{s}_{33300}=\tilde{s}_{33330}, \tilde{s}_{30000}+\tilde{s}_{33330}=\tilde{s}_{33333}, \tilde{s}_{31000}+\tilde{s}_{32000}=\tilde{s}_{33310}, \tilde{s}_{31000}+\tilde{s}_{33200}=\tilde{s}_{33331}, \tilde{s}_{31000}+\tilde{s}_{33320}=\tilde{s}_{33333}$, $\tilde{s}_{31100}+\tilde{s}_{33220}=\tilde{s}_{33333}, \tilde{s}_{31110}+\tilde{s}_{32220}=\tilde{s}_{33333}, 2 \tilde{s}_{32000}=\tilde{s}_{33320}, \tilde{s}_{32000}+\tilde{s}_{33100}=\tilde{s}_{33331}, \tilde{s}_{32000}+\tilde{s}_{33200}=\tilde{s}_{33332}$, $\tilde{s}_{32000}+\tilde{s}_{33310}=\tilde{s}_{33333}, 2 \tilde{s}_{32100}=\tilde{s}_{33332}, \tilde{s}_{32100}+\tilde{s}_{33210}=\tilde{s}_{33333}, \tilde{s}_{32110}+\tilde{s}_{32210}=\tilde{s}_{33333}, \tilde{s}_{32200}+\tilde{s}_{33110}=\tilde{s}_{33333}$, $2 \tilde{s}_{33000}=\tilde{s}_{33330}, \tilde{s}_{33000}+\tilde{s}_{33300}=\tilde{s}_{33333}$ and $\tilde{s}_{33100}+\tilde{s}_{33200}=\tilde{s}_{33333}$.

Moreover, we have that $\tilde{s}_{00000}=s_{0}=0, \tilde{s}_{11111}=s_{1}=1 / 3, \tilde{s}_{22222}=s_{2}=2 / 3$ and $\tilde{s}_{33333}=s_{3}=1$. It appears that the system of linear equations is not complete with $\tilde{s}_{31110}$ as a parameter $p$ such that $17 / 42 \leq p \leq 16 / 35$ (by 
the isotonicity of $\tilde{s}$ ). To supplement the system, we are looking for a consistent equation by applying $\tilde{s}$ to suitable deflected partial products. We choose the equations:

$$
\tilde{s}_{11100}+\tilde{s}_{20000}=\tilde{s}_{31110}
$$

The system of linear equations together with this complementary equation yield the required solutions. Finally, applying $\tilde{s}$ to all deflected partial products, we complete the proof.

Observe that the 4-extension $\mathcal{W}_{3}^{\mathcal{N}_{4}}$ of the Weber $M V$-chain $\mathcal{W}_{3}$ contains the following four Weber $M V$-chains:

$00000<10000<10000^{2 \dot{\varphi}}(=21000)<10000^{3 \dot{\phi}}(=32100)<10000^{4 \dot{\varphi}}(=33210)<10000^{5 \dot{\varphi}}(=33321)<$ $10000^{6 \dot{\varphi}}(=33332)<10000^{7 \dot{\phi}}(=33333), 00000<11111<11111^{2 \dot{\varphi}}(=22222)<11111^{3 \dot{\varphi}}(=33333), 00000<$ $30000<30000^{2 \dot{\phi}}(=33000)<30000^{3 \dot{\varphi}}(=33300)<30000^{4 \dot{\varphi}}(=33330)<30000^{5 \dot{\phi}}(=33333)$ and $00000<$ $21100<21100^{2 \dot{\varphi}}(=33221)<21100^{3 \dot{\phi}}(=33333)$.

We emphasize that PM of $\tilde{s}$ cases to exist on the partial binary product $21100 \wp 21100=33221$. This fact opposes an axiom of Weber states.

\section{Copyrights}

Copyright for this article is retained by the author(s), with first publication rights granted to the journal.

This is an open-access article distributed under the terms and conditions of the Creative Commons Attribution license (http://creativecommons.org/licenses/by/3.0/). 